\title{
Au-Catalyzed Cyclization of Monoallylic Diols
}

\author{
Aaron Aponick,* Chuan-Ying Li, and Berenger Biannic \\ Department of Chemistry, University of Florida, Gainesville, Florida 32611 \\ aponick@chem.ufl.edu
}

\section{General:}

\section{Supporting Information}

All reactions were carried out under an atmosphere of nitrogen unless otherwise specified. Anhydrous solvents were transferred via syringe to flame-dried glassware, which had been cooled under a stream of dry nitrogen. Anhydrous tetrahydrofuran (THF), acetonitrile, ether, dichloromethane, pentane were dried using a mBraun solvent purification system.

Analytical thin layer chromatography (TLC) was performed using $250 \mu \mathrm{m}$ Silica Gel $60 \mathrm{~F}_{254}$ pre-coated plates (EMD Chemicals Inc.). Flash column chromatography was performed using 230-400 Mesh 60A Silica Gel (Whatman Inc.). The eluents employed are reported as volume:volume percentages. Proton nuclear magnetic resonance $\left({ }^{1} \mathrm{H}\right.$ NMR) spectra were recorded using Varian Unity Inova $500 \mathrm{MHz}$ and Varian Mercury $300 \mathrm{MHz}$ spectrometers. Chemical shift $(\delta)$ is reported in parts per million (ppm) downfield relative to tetramethylsilane (TMS, $0.0 \mathrm{ppm}$ ) or $\mathrm{CDCl}_{3}(7.26 \mathrm{ppm})$. Coupling constants $(J)$ are reported in $\mathrm{Hz}$. Multiplicities are reported using the following abbreviations: s, singlet; d, doublet; t, triplet; q, quartet; m, multiplet; br, broad; Carbon-13 nuclear magnetic resonance $\left({ }^{13} \mathrm{C}\right.$ NMR) spectra were recorded using a Varian Unity Mercury 300 spectrometer at $75 \mathrm{MHz}$. Chemical shift is reported in ppm relative to the carbon resonance of $\mathrm{CDCl}_{3}(77.00 \mathrm{ppm})$. Infrared spectra were obtained on a Bruker Vector 22 IR spectrometer at $4.0 \mathrm{~cm}^{-1}$ resolution and are reported in wavenumbers. High resolution mass spectra (HRMS) were obtained by Mass Spectrometry Core Laboratory of University of Florida, and are reported as $\mathrm{m} / \mathrm{e}$ (relative ratio). Accurate masses are reported for the molecular ion $(\mathrm{M}+)$ or a suitable fragment ion.

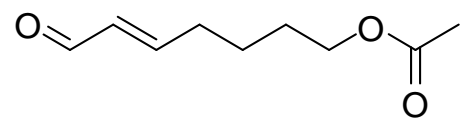

(E)-7-oxohept-5-enyl acetate (4a). A solution of hex-5-enyl acetate (170.1 $\mathrm{mg}, 1 \mathrm{mmol})$ and crotonaldehyde $(350.1 \mathrm{mg}, 5 \mathrm{mmol})$ in dry $\mathrm{CH}_{2} \mathrm{Cl}_{2}(2 \mathrm{~mL})$ was added to a solution of Grubbs $2^{\text {nd }}$ generation catalyst $(25.5 \mathrm{mg}, 0.03 \mathrm{mmol}, 3 \mathrm{~mol} \%)$ in dry $\mathrm{CH}_{2} \mathrm{Cl}_{2}(3 \mathrm{~mL})$. The mixture was stirred at reflux for 2 hours and then cooled to rt. Silica gel (200 mg) was 
added and the reaction mixture was stirred open to air for $30 \mathrm{~min}$. The solvent was removed and the crude product was purified by flash chromatography $(50 \%$ EtOAc/Hexanes) to give the product as a yellow oil (116.8 $\mathrm{mg}, 92 \%)$ that satisfactorily matched all previously reported data. ${ }^{1}$

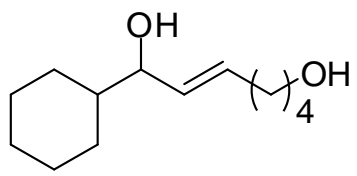

(E)-1-cyclohexylhept-2-ene-1,7-diol (4). A solution of cyclohexylmagnesium bromide (2 $\mathrm{M}$ in $\mathrm{Et}_{2} \mathrm{O}, 1.120 \mathrm{~mL}, 3.3$ eq.) was added dropwise to a solution of $\mathbf{4 a}(100 \mathrm{mg}, 0.78$ $\mathrm{mmol})$ in dry THF $(6 \mathrm{~mL})$ at $-78^{\circ} \mathrm{C}$. The mixture was stirred $2 \mathrm{~h}$ and then quenched with $\mathrm{NH}_{4} \mathrm{Cl}(6 \mathrm{~mL}$ of a saturated aqueous solution), diluted with water $(30 \mathrm{~mL})$ and extracted with $\mathrm{CH}_{2} \mathrm{Cl}_{2}(3 \times 20 \mathrm{~mL})$. The combined organic layers were dried over $\mathrm{MgSO}_{4}$, concentrated, and purified by flash chromatography (30\% EtOAc/Hexanes) to give the product as a colorless oil $(146.9 \mathrm{mg}, 71 \%) . \mathrm{R}_{\mathrm{f}}=0.12$ (20\% EtOAc/hexanes); IR (neat) $3356,2924,2852,1449,1003,433 \mathrm{~cm}^{-1}$; ${ }^{1} \mathrm{H}$ NMR $\left(300 \mathrm{MHz}, \mathrm{CDCl}_{3}\right): \delta 5.59(\mathrm{dt}, J=6.3$, $15.3 \mathrm{~Hz}, 1 \mathrm{H}), 5.45(\mathrm{dd}, J=6.9,15.3 \mathrm{~Hz}, 1 \mathrm{H}), 3.75(\mathrm{t}, J=7.2 \mathrm{~Hz}, 1 \mathrm{H}), 3.63$ (t, $J=6 \mathrm{~Hz}$, $2 \mathrm{H}), 2.06(\mathrm{q}, J=6.9 \mathrm{~Hz}, 2 \mathrm{H}), 1.86-0.88(\mathrm{~m}, 18 \mathrm{H}) ;{ }^{13} \mathrm{C} \mathrm{NMR}\left(75 \mathrm{MHz}, \mathrm{CDCl}_{3}\right): \delta 132.7$, $132.1,77.84,62.93,43.88,32.40,32.18,29.0,28.9,26.7,26.3,26.2,25.6$; HRMS (ESI) Calcd for $\mathrm{C}_{13} \mathrm{H}_{23} \mathrm{O}_{2}(\mathrm{M}-\mathrm{H})^{+}$211.1693, found 211.1704.

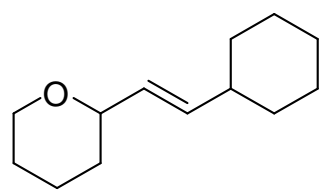

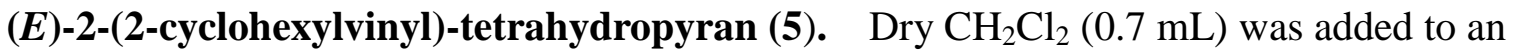
aluminum foil covered test tube containing $\mathrm{PPh}_{3} \mathrm{AuCl}(1.3 \mathrm{mg}, 0.003 \mathrm{mmol}), \mathrm{AgOTf}(0.7$ $\mathrm{mg}, 0.003 \mathrm{mmol})$ and activated MS-4 $\AA$ ( $25 \mathrm{mg})$. After stirring for 10 minutes, a solution of diol $4(56.1 \mathrm{mg}, 0.26 \mathrm{mmol})$ in dry $\mathrm{CH}_{2} \mathrm{Cl}_{2}(0.7 \mathrm{~mL})$ was added. After TLC analysis showed the reaction to be complete (40 min), it was diluted with $\mathrm{CH}_{2} \mathrm{Cl}_{2}$ and filtered through a short plug of silica. The solution of crude product was concentrated, and then purified by flash chromatography (5\% EtOAc/hexanes) to give the product as a colorless oil (48.6 mg, 96\%). $\mathrm{R}_{\mathrm{f}}=0.81$ (5\% EtOAc/hexanes); IR (neat) 2925, 2851, 1448, 1085, $968,412 \mathrm{~cm}^{-1} ; \quad{ }^{1} \mathrm{H}$ NMR $\left(300 \mathrm{MHz}, \mathrm{CDCl}_{3}\right): \delta 5.59(\mathrm{dd}, J=6.3,15.3 \mathrm{~Hz}, 1 \mathrm{H}), 5.39$ $(\mathrm{dd}, 6.3,15.3 \mathrm{~Hz}, 1 \mathrm{H}), 3.98(\mathrm{dt}, J=2.7,10.8 \mathrm{~Hz}, 1 \mathrm{H}), 3.70(\mathrm{dd}, J=6.0,10.5 \mathrm{~Hz}, 1 \mathrm{H})$, $3.45(\mathrm{dt}, J=2.4,11.7 \mathrm{~Hz}, 1 \mathrm{H}), 1.95-0.97(\mathrm{~m}, 17 \mathrm{H}) ;{ }^{13} \mathrm{C} \mathrm{NMR}\left(75 \mathrm{MHz}, \mathrm{CDCl}_{3}\right): \delta 137.8$, 128.9, 78.7, 68.6, 40.5, 32.9, 33.0, 32.5, 26.4, 26.3, 26.1, 23.7; HRMS (ESI) Calcd for $\mathrm{C}_{13} \mathrm{H}_{23} \mathrm{O}(\mathrm{M}+\mathrm{H})^{+}$195.1754, found 195.1749. 


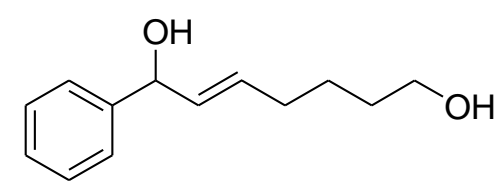

(E)-1-phenylhept-2-ene-1,7-diol (6). To a solution of Grubb's catalyst $2^{\text {nd }}$ generation (3 mol\%, $25.5 \mathrm{mg})$ in dry $\mathrm{CH}_{2} \mathrm{Cl}_{2}(3 \mathrm{~mL})$ in a flamed dried flask was added solution of 1-phenylprop-2-en-1-ol (134.0 mg, $1 \mathrm{mmol})$ and hex-5-enyl acetate $(284.0 \mathrm{mg}, 2 \mathrm{mmol})$ in dry $\mathrm{CH}_{2} \mathrm{Cl}_{2}(2 \mathrm{~mL})$. The mixture was stirred at reflux for 6 hours, cooled to r.t, and then filtered through a short plug of silica. The solvent was removed and the crude product was purified by flash chromatography $(15 \%$ EtOAc/Hexanes $)$ to give (E)-7-hydroxy-7-phenylhept-5-enyl acetate as a colorless oil(109 mg, 44\%).

The oil was dissolved in $3 \mathrm{~mL} \mathrm{MeOH}$, and then mixed with $1 \mathrm{~mL}$ aqueous $\mathrm{K}_{2} \mathrm{CO}_{3}$ (91 mg, $0.66 \mathrm{mmol}$ ). The mixture stirred at room temperature for $9 \mathrm{~h}$, diluted with $10 \mathrm{~mL}$ water, extracted with $\mathrm{CH}_{2} \mathrm{Cl}_{2}(3 \times 10 \mathrm{~mL})$, The organic layer was dried over $\mathrm{Na}_{2} \mathrm{SO}_{4}$, concentrated and then purified by flash chromatography (30\% EtOAc/Hexanes) to give a colorless oil (57 mg, 63\%). IR (neat) 3356, 3029, 2933, 1453, $970 \mathrm{~cm}^{-1} .{ }^{1} \mathrm{H}$ NMR (300 $\left.\mathrm{MHz}, \mathrm{CDCl}_{3}\right) \delta$ 7.34-7.24 (m, 5H), 5.76-5.59 (m, 2H), $5.11(\mathrm{~d}, J=5.1 \mathrm{~Hz}, 1 \mathrm{H}), 3.55(\mathrm{t}, J$ $=5.7 \mathrm{~Hz}, 2 \mathrm{H}), 2.05(\mathrm{q}, J=6.3 \mathrm{~Hz}, 2 \mathrm{H}), 1.74(\mathrm{br} \mathrm{s}, 1 \mathrm{H}), 1.55-1.39(\mathrm{~m}, 4 \mathrm{H}) .{ }^{13} \mathrm{C}$ NMR $(75$ $\left.\mathrm{MHz}, \mathrm{CDCl}_{3}\right) \delta 143.3,132.6,132.0,128.4,127.4,126.1,75.0,62.6,32.1,31.8,25.1$. HRMS (ESI) Calcd for $\mathrm{C}_{13} \mathrm{H}_{18} \mathrm{NaO}_{2}(\mathrm{M}+\mathrm{Na})^{+} 229.1199$, found 229.1213 .

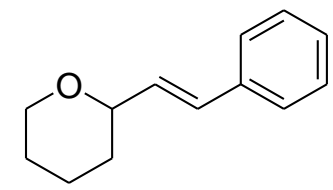

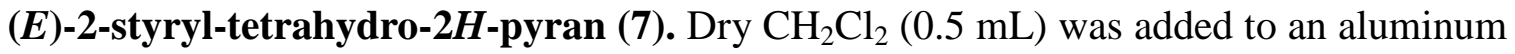
foil covered test tube containing $\mathrm{PPh}_{3} \mathrm{AuCl}(1.0 \mathrm{mg}, 0.002 \mathrm{mmol})$, AgOTf $(0.5 \mathrm{mg}, 0.002$

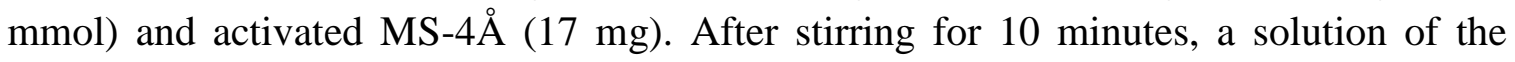
(E)-1-phenylhept-2-ene-1,7-diol $6(43.0 \mathrm{mg}, 0.2 \mathrm{mmol})$ in dry $\mathrm{CH}_{2} \mathrm{Cl}_{2}(0.5 \mathrm{~mL})$ was added. The reaction mixture was stirred at r.t. under nitrogen for 30 minutes. After TLC analysis showed the reaction to be complete it was diluted with $\mathrm{CH}_{2} \mathrm{Cl}_{2}$ and filtered through a short plug of silica. The solution was concentrated, and the crude product purified by flash chromatography ( $2 \%$ EtOAc/hexanes) to give the product as a colorless oil (35.0 mg, $89 \%)$ that satisfactorily matched all previously reported data. ${ }^{2}$

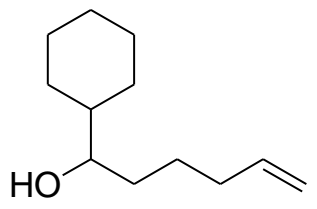

1-cyclohexylhex-5-en-1-ol (8a). To a solution of cyclohexane carbaldehyde ( 1.12 g, 10 mmol $)$ in dry $\mathrm{THF}(20 \mathrm{~mL})$ at $-78^{\circ} \mathrm{C}$ was added dropwise a solution of pent-4-enylmagnesium bromide (prepared from 5-bromopent-1-ene (1.49 g, 10.mmol), 
$\mathrm{Mg}(252 \mathrm{mg}, 10.5 \mathrm{mmol})$, THF $(20 \mathrm{~mL})$ ). The mixture was stirred at $-78^{\circ} \mathrm{C}$ for $60 \mathrm{~min}$ and then quenched with a saturated aqueous solution of $\mathrm{NH}_{4} \mathrm{Cl}(20 \mathrm{~mL})$. After separation, the aqueous layer was extracted with $\mathrm{CH}_{2} \mathrm{Cl}_{2}(3 \times 20 \mathrm{~mL})$. The organic layer was dried over $\mathrm{MgSO}_{4}$ and then purified by flash chromatography (10\% EtOAc/Hexanes) to give the product as a colorless oil $(1.0 \mathrm{~g}, 55 \%)$ that satisfactorily matched all previously reported data. ${ }^{3 a}$

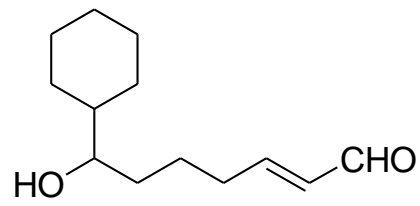

(E)-7-cyclohexyl-7-hydroxyhept-2-enal $(\mathbf{8 b})$. To a solution of Grubb's catalyst $2^{\text {nd }}$ generation $(3 \mathrm{~mol} \%, 25.5 \mathrm{mg})$ in dry $\mathrm{CH}_{2} \mathrm{Cl}_{2}(3 \mathrm{~mL})$ in a flamed dried flask was added solution of 1-cyclohexylhex-5-en-1-ol 8a (182.2 mg, $1 \mathrm{mmol})$ and crotonaldehyde (350.5 $\mathrm{mg}, 5 \mathrm{mmol})$ in dry $\mathrm{CH}_{2} \mathrm{Cl}_{2}(2 \mathrm{~mL})$. The mixture was stirred at reflux for 11 hours, cooled to r.t. and then filtered through a short plug of silica. The solvent was removed and the crude product was purified by flash chromatography (10\% EtOAc/Hexanes) to give the product as a colorless oil $(166 \mathrm{mg}, 79 \%) .{ }^{1} \mathrm{H}$ NMR $\left(300 \mathrm{MHz}, \mathrm{CDCl}_{3}\right) \delta 9.50(\mathrm{~d}, J=8.1$ $\mathrm{Hz}, 1 \mathrm{H}), 6.85$ (dt, $J=15.6,6.6 \mathrm{~Hz}, 1 \mathrm{H}), 6.12$ (ddt, $J=15.6,8.1,1.5 \mathrm{~Hz}, 1 \mathrm{H}), 3.39-3.33$ $(\mathrm{m}, 1 \mathrm{H}), 2.40-2.32(\mathrm{~m}, 2 \mathrm{H}), 1.80-0.91(\mathrm{~m}, 16 \mathrm{H}) .{ }^{13} \mathrm{C}$ NMR $\left(75 \mathrm{MHz}, \mathrm{CDCl}_{3}\right) \delta$ 194.0, $158.8,132.8,75.5,43.6,33.2,32.6,29.0,27.7,26.3,26.1,26.0,24.1$.

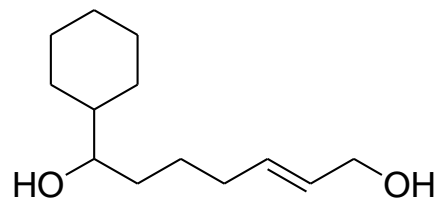
(E)-7-cyclohexylhept-2-ene-1,7-diol
(8).
To
a solution
of

(E)-7-cyclohexyl-7-hydroxyhept-2-enal $\mathbf{8 b}(166 \mathrm{mg}, 0.79 \mathrm{mmol})$ in $\mathrm{MeOH}(4 \mathrm{~mL})$ at $0^{\circ} \mathrm{C}$

was added $\mathrm{NaBH}_{4}(36 \mathrm{mg}, 0.95 \mathrm{mmol})$ over 1 minute. After stirring at $0^{\circ} \mathrm{C}$ for 30 minutes, a saturated aqueous solution of $\mathrm{NH}_{4} \mathrm{Cl}(2 \mathrm{~mL})$ was added, and then $10 \mathrm{~mL}$ water. The aqueous layer was extracted with $\mathrm{CH}_{2} \mathrm{Cl}_{2}(3 \times 10 \mathrm{~mL})$. The combined organic extract was dried over $\mathrm{MgSO}_{4}$ and then purified by flash chromatography (30\% EtOAc/Hexanes) to give the product as a colorless oil (145 mg, 86\%). IR (neat) 3383, 2924, 1670, 1449, 969. ${ }^{1} \mathrm{H}$ NMR $\left(300 \mathrm{MHz}, \mathrm{CDCl}_{3}\right)$ 8 5.74-5.58 (m, 2H), $4.07(\mathrm{~d}, J=4.5 \mathrm{~Hz}, 2 \mathrm{H}), 3.36-3.32((\mathrm{~m}$, $1 \mathrm{H}), 2.10-2.03(\mathrm{~m}, 2 \mathrm{H}), 1.80-0.95(\mathrm{~m}, 17 \mathrm{H}) .{ }^{13} \mathrm{C} \mathrm{NMR}\left(75 \mathrm{MHz}, \mathrm{CDCl}_{3}\right) \delta 132.5,129.2$, 75.8, 63.2, 43.5, 33.3, 32.1, 29.1, 27.7, 26.4, 26.2, 26.1, 25.3. HRMS (ESI) Calcd for $\mathrm{C}_{13} \mathrm{H}_{24} \mathrm{NaO}_{2}(\mathrm{M}+\mathrm{Na})^{+} 235.1669$, found 235.1649. 


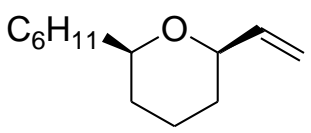

Cis-2-cyclohexyl-6-vinyl-tetrahydro-2H-pyran (9). ${ }^{3 \mathrm{~b}}$ Dry $\mathrm{CH}_{2} \mathrm{Cl}_{2}(0.5 \mathrm{~mL})$ was added to an aluminum foil covered test tube containing $\mathrm{PPh}_{3} \mathrm{AuCl}(1.0 \mathrm{mg}, 0.002 \mathrm{mmol})$, $\operatorname{AgOTf}(0.5 \mathrm{mg}, 0.002 \mathrm{mmol})$ and activated MS-4 $\AA$ (17 mg). After stirring for 10 minutes, the mixture was cooled to $-50^{\circ} \mathrm{C}$ and a solution of diol $8(42.4 \mathrm{mg}, 0.2 \mathrm{mmol})$ in dry $\mathrm{CH}_{2} \mathrm{Cl}_{2}(0.5 \mathrm{~mL})$ was added. After TLC analysis showed the reaction to be complete $(8 \mathrm{~h})$, it was diluted with $\mathrm{CH}_{2} \mathrm{Cl}_{2}$ and filtered through a short plug of silica. The solution of crude product was concentrated, and then purified by flash chromatography (1\% EtOAc/hexanes) to give the product as a colorless oil $(30.8 \mathrm{mg}, 80 \%)$. Data for major product (Cis): IR (neat) 2926, 2853, 1645, 1450, 1076, 918. ${ }^{1} \mathrm{H}$ NMR (300 MHz, $\mathrm{CDCl}_{3}$ ) $\delta 5.86(\mathrm{ddd}, J=17.4,10.8,5.1 \mathrm{~Hz}, 1 \mathrm{H}), 5.23(\mathrm{dt}, J=17.4,1.8 \mathrm{~Hz}, 1 \mathrm{H}), 5.05(\mathrm{dt}, J=10.8$, $1.8 \mathrm{~Hz}, 1 \mathrm{H}), 3.80-3.73(\mathrm{~m}, 1 \mathrm{H}), 3.09-3.02(\mathrm{~m}, 1 \mathrm{H}), 1.98-1.71(\mathrm{~m}, 2 \mathrm{H}), 1.63-0.96(\mathrm{~m}$, $15 \mathrm{H}) .{ }^{13} \mathrm{C} \mathrm{NMR}\left(75 \mathrm{MHz}, \mathrm{CDCl}_{3}\right) \delta 139.9,113.9,82.1,78.2,43.2,31.7,29.3,28.7,27.9$, 26.7, 26.3, 26.2, 23.7. HRMS (ESI) Calcd for $\mathrm{C}_{13} \mathrm{H}_{22} \mathrm{NaO}(\mathrm{M}+\mathrm{Na})^{+} 217.1563$, found 217.1571.

The relative configuration of the major diastereomer was determined by NOE DIFF experiments as follows:
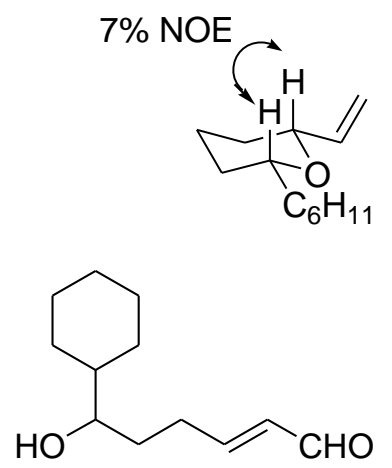

(E)-6-cyclohexyl-6-hydroxyhex-2-enal (10a). To a solution of cyclohexane carbaldehyde $(1.68 \mathrm{~g}, 15 \mathrm{mmol})$ in dry THF $(30 \mathrm{~mL})$ at $-78^{\circ} \mathrm{C}$ was added dropwise a solution of but-3-enylmagnesium bromide (prepared from 4-bromobut-1-ene (2.03 g, 15 $\mathrm{mmol}), \mathrm{Mg}(380 \mathrm{mg}, 15.8 \mathrm{mmol})$, THF $(20 \mathrm{~mL}))$. The mixture was stirred at $-78^{\circ} \mathrm{C}$ for 30 min and then quenched with a saturated aqueous solution of $\mathrm{NH}_{4} \mathrm{Cl}(30 \mathrm{~mL})$. After separation, the aqueous layer was extracted with $\mathrm{CH}_{2} \mathrm{Cl}_{2}(3 \times 20 \mathrm{~mL})$. The combined organic extract was dried over $\mathrm{MgSO}_{4}$ and then purified by flash chromatography $(10 \%$ EtOAc/Hexanes) to give 1-cyclohexylpent-4-en-1-ol as a colorless oil (1.40 g, 55\%).

To a solution of Grubb's catalyst $2^{\text {nd }}$ generation $(3 \mathrm{~mol} \%, 51.0 \mathrm{mg})$ in dry $\mathrm{CH}_{2} \mathrm{Cl}_{2}(6$ $\mathrm{mL}$ ) in a flamed dried flask was added solution of 1-cyclohexylpent-4-en-1-ol (336 mg, 2 $\mathrm{mmol}$ ) and crotonaldehyde (700.9 $\mathrm{mg}, 10 \mathrm{mmol})$ in dry $\mathrm{CH}_{2} \mathrm{Cl}_{2}(4 \mathrm{~mL})$. The mixture was stirred at reflux for 11 hours, cooled to r.t. and then filtered through a short plug of silica. 
The solvent was removed and the crude was purified by flash chromatography (10\% EtOAc/Hexanes) to give the product as a colorless oil (242 mg, 62\%). ${ }^{1} \mathrm{H}$ NMR (300 $\left.\mathrm{MHz}, \mathrm{CDCl}_{3}\right) \delta 9.47(\mathrm{~d}, J=7.8 \mathrm{~Hz}, 1 \mathrm{H}), 6.88(\mathrm{dt}, J=15.6,6.6 \mathrm{~Hz}, 1 \mathrm{H}), 6.11$ (ddt, $J=$ 15.6, 7.8, $1.2 \mathrm{~Hz}, 1 \mathrm{H}), 3.38-3.32(\mathrm{~m}, 1 \mathrm{H}), 2.60-2.31(\mathrm{~m}, 2 \mathrm{H}), 1.80-0.92(\mathrm{~m}, 13 \mathrm{H}) .{ }^{13} \mathrm{C}$ NMR $\left(75 \mathrm{MHz}, \mathrm{CDCl}_{3}\right) \delta 194.0,158.8,132.9,75.3,43.8,32.1,29.3,29.1,27.8,26.4$, 26.2, 26.0.

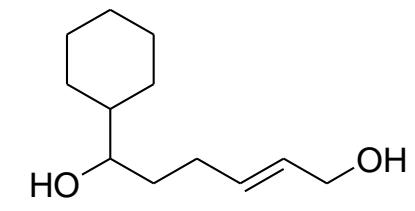
(E)-6-cyclohexylhex-2-ene-1,6-diol
(10).
To
a solution
of

(E)-6-cyclohexyl-6-hydroxyhex-2-enal 10a $(242 \mathrm{mg}, 1.23 \mathrm{mmol})$ in $\mathrm{MeOH}(6 \mathrm{~mL})$ at $0^{\circ} \mathrm{C}$

was added $\mathrm{NaBH}_{4}(57 \mathrm{mg}, 1.5 \mathrm{mmol})$ over 1 minute. After stirring at $0^{\circ} \mathrm{C}$ for 30 minutes, a saturated aqueous solution of $\mathrm{NH}_{4} \mathrm{Cl}(3 \mathrm{~mL})$ was added, and then $15 \mathrm{~mL}$ water. The aqueous layer was extracted with $\mathrm{CH}_{2} \mathrm{Cl}_{2}(3 \times 15 \mathrm{~mL})$. The combined organic extract was dried over $\mathrm{MgSO}_{4}$ and then purified by flash chromatography (30\% EtOAc/Hexanes) to give the product as a colorless oil (213 mg, 88\%). IR (neat) 3332, 2924, 1670, 1449, 969. ${ }^{1} \mathrm{H}$ NMR $\left(300 \mathrm{MHz}, \mathrm{CDCl}_{3}\right) \delta 5.74-5.58(\mathrm{~m}, 2 \mathrm{H}), 4.04(\mathrm{~d}, J=4.5 \mathrm{~Hz}, 2 \mathrm{H}), 3.36-3.30(\mathrm{~m}$, $1 \mathrm{H}), 2.26-2.04(\mathrm{~m}, 2 \mathrm{H}), 1.75-0.94(\mathrm{~m}, 13 \mathrm{H}) .{ }^{13} \mathrm{C} \mathrm{NMR}\left(75 \mathrm{MHz}, \mathrm{CDCl}_{3}\right) \delta 132.7,129.3$, 75.5, 63.4, 43.7, 33.4, 29.1, 28.7, 27.8, 26.5, 26.3, 26.1. HRMS (ESI) Calcd for $\mathrm{C}_{12} \mathrm{H}_{22} \mathrm{NaO}_{2}(\mathrm{M}+\mathrm{Na})^{+}$221.1512, found 221.1519.

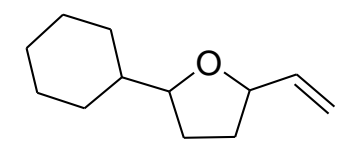

2-cyclohexyl-5-vinyl-tetrahydrofuran (11). Dry $\mathrm{CH}_{2} \mathrm{Cl}_{2}(0.5 \mathrm{~mL})$ was added to an aluminum foil covered test tube containing $\mathrm{PPh}_{3} \mathrm{AuCl}$ ( $\left.1.0 \mathrm{mg}, 0.002 \mathrm{mmol}\right), \mathrm{AgOTf}(0.5$ $\mathrm{mg}, 0.002 \mathrm{mmol})$ and activated MS-4 $\AA$ (17 mg). After stirred for 10 minutes, cooled to $-50^{\circ} \mathrm{C}$, a solution of diol $10(39.6 \mathrm{mg}, 0.2 \mathrm{mmol})$ in dry $\mathrm{CH}_{2} \mathrm{Cl}_{2}(0.5 \mathrm{~mL})$ was added. After TLC analysis showed the reaction to be complete $\left(10 \mathrm{~h}\right.$ ), it was diluted with $\mathrm{CH}_{2} \mathrm{Cl}_{2}$ and filtered through a short plug of silica. The solution of crude product was concentrated, and then purified by flash chromatography ( $2 \% \mathrm{EtOAc/hexanes)} \mathrm{to} \mathrm{give} \mathrm{the} \mathrm{product} \mathrm{as} \mathrm{a}$ colorless oil (28.4 mg, $79 \%$ ). Data for major product (Cis): IR (neat) 3080, 2924, 2853, $1449,1055,950 .{ }^{1} \mathrm{H}$ NMR $\left(300 \mathrm{MHz}, \mathrm{CDCl}_{3}\right) \delta 5.83(\mathrm{ddd}, J=17.4,10.5,6.6 \mathrm{~Hz}, 1 \mathrm{H})$, $5.20(\mathrm{dt}, J=17.4,1.5 \mathrm{~Hz}, 1 \mathrm{H}), 5.05(\mathrm{dt}, J=10.5,1.5 \mathrm{~Hz}, 1 \mathrm{H}), 4.32(\mathrm{q}, J=7.0 \mathrm{~Hz}, 1 \mathrm{H})$, $3.68(\mathrm{q}, J=7.2 \mathrm{~Hz}, 1 \mathrm{H}), 2.09-0.90(\mathrm{~m}, 15 \mathrm{H}) .{ }^{13} \mathrm{C} \mathrm{NMR}\left(75 \mathrm{MHz}, \mathrm{CDCl}_{3}\right) \delta 139.7,114.7$, 83.8, 79.6, 43.2, 32.7, 29.9, 29.6, 28.8, 26.6, 26.1, 26.0. HRMS (ESI) Calcd for $\mathrm{C}_{12} \mathrm{H}_{19}$ $\left(\mathrm{M}+\mathrm{H}-\mathrm{H}_{2} \mathrm{O}\right)^{+} 163.1481$, found 163.1494 .

The relative configuration of the major diastereomer was determined by NOE DIFF 
experiments as follows:

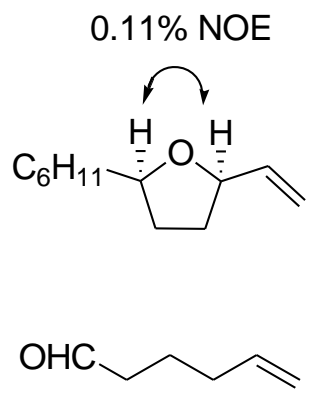

Hex-5-enal (12a). ${ }^{4}$ To a solution of 5-hexen-1-ol $(0.9 \mathrm{~mL}, 7.49 \mathrm{mmol})$ in $\mathrm{CH}_{2} \mathrm{Cl}_{2}(12 \mathrm{~mL})$ was added pyridinium chlorochromate $(2.45 \mathrm{~g}, 11.4 \mathrm{mmol})$. The resulting dark red solution was stirred for $14 \mathrm{~h}$ at room temperature, diluted with pentane $(12 \mathrm{~mL})$, filtered through a plug of silica with 1:1 $\mathrm{CH}_{2} \mathrm{Cl}_{2} /$ pentane $(7 \times 25 \mathrm{~mL})$. The filtrate was concentrated to give 5-hexen-1-al as a clear, colorless oil. The unpurified aldehyde was carried immediately into the next step without further purification.

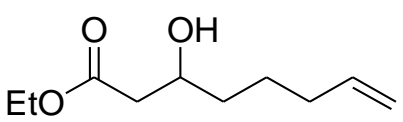

Ethyl 3-hydroxyoct-7-enoate (12b). To a solution of $i$ - $\mathrm{Pr}_{2} \mathrm{NH}(2.2 \mathrm{~mL}, 15.8 \mathrm{mmol})$ in dry THF $(20 \mathrm{~mL})$ was added $n$-BuLi $\left(6 \mathrm{~mL}, 2.5 \mathrm{M}\right.$ in hexane) at $-78^{\circ} \mathrm{C}$. The mixture was kept at this temperature for $1 \mathrm{~h}$, then a mixture of ethyl acetate $(15 \mathrm{mmol})$ and dry THF $(10 \mathrm{~mL})$ was added at low temperature. After stirring for $1 \mathrm{~h}$ at $-78^{\circ} \mathrm{C}$, a mixture of Hex-5-enal 12a $(7.5 \mathrm{mmol})$ and dry THF $(10 \mathrm{~mL})$ was added. After the mixture was stirred for an additional $1 \mathrm{~h}$, saturated $\mathrm{NH}_{4} \mathrm{Cl}$ solution was added and the aqueous layer was extracted with ethyl acetate $(3 \times 30 \mathrm{~mL})$, the combined extracts were dried and evaporated in vacuum. The residue was subjected to flash chromatography to furnish the product as a colorless oil (761 mg, 55\% for two steps) that satisfactorily matched all previously reported data. ${ }^{5}$<smiles>CCOC(=O)CC(O)CCC/C=C/C=O</smiles>

(E)-ethyl 3-hydroxy-9-oxonon-7-enoate (12c). To a solution of Grubb's catalyst $2^{\text {nd }}$ generation $(3 \mathrm{~mol} \%, 51.0 \mathrm{mg})$ in dry $\mathrm{CH}_{2} \mathrm{Cl}_{2}(6 \mathrm{~mL})$ in a flamed dried flask was added solution of ethyl 3-hydroxyoct-7-enoate $\mathbf{1 2 b}(372 \mathrm{mg}, 2 \mathrm{mmol})$ and crotonaldehyde (700.9 $\mathrm{mg}, 10 \mathrm{mmol})$ in dry $\mathrm{CH}_{2} \mathrm{Cl}_{2}(4 \mathrm{~mL})$. The mixture was stirred at reflux for 11 hours, cooled to r.t. and then filtered through a short plug of silica. The solvent was removed and the crude was purified by flash chromatography (30\% EtOAc/Hexanes) to give the product as a colorless oil (411 mg, 96\%). ${ }^{1} \mathrm{H}$ NMR $\left(300 \mathrm{MHz}, \mathrm{CDCl}_{3}\right) \delta 9.37(\mathrm{~d}$, $J=7.8 \mathrm{~Hz}, 1 \mathrm{H}), 6.76(\mathrm{dt}, J=15.6,6.9 \mathrm{~Hz}, 1 \mathrm{H}), 6.00(\mathrm{ddt}, J=15.6,7.8,1.5 \mathrm{~Hz}, 1 \mathrm{H}), 4.04$ 
(q, $J=6.9 \mathrm{~Hz}, 2 \mathrm{H}), 3.95-3.87(\mathrm{~m}, 1 \mathrm{H}), 3.34(\mathrm{br} \mathrm{s}, 1 \mathrm{H}), 2.36-2.23(\mathrm{~m}, 4 \mathrm{H}), 1.63-1.35(\mathrm{~m}$, $4 \mathrm{H}), 1.15(\mathrm{t}, J=6.9 \mathrm{~Hz}, 3 \mathrm{H}) .{ }^{13} \mathrm{C} \mathrm{NMR}\left(75 \mathrm{MHz}, \mathrm{CDCl}_{3}\right) \delta 193.8,172.4,158.2,132.8$, 67.2, 60.4, 41.3, 35.6, 32.1, 23.5, 13.9.

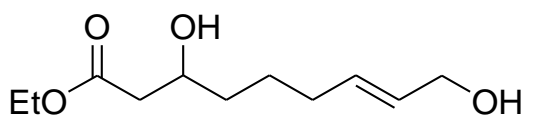

(E)-ethyl 3,9-dihydroxynon-7-enote (12). To a solution of (E)-ethyl 3-hydroxy-9-oxonon-7-enoate $12 \mathrm{c}(411 \mathrm{mg}, 1.92 \mathrm{mmol})$ in $\mathrm{MeOH}(8 \mathrm{~mL})$ at $0^{\circ} \mathrm{C}$ was added $\mathrm{NaBH}_{4}\left(87 \mathrm{mg}, 2.3 \mathrm{mmol}\right.$ ) over 1 minute, After stirred at $0^{\circ} \mathrm{C}$ for 30 minutes, a saturated aqueous solution of $\mathrm{NH}_{4} \mathrm{Cl}(4 \mathrm{~mL})$ was added, and then $15 \mathrm{~mL}$ water. The aqueous layer was extracted with $\mathrm{CH}_{2} \mathrm{Cl}_{2}(3 \times 15 \mathrm{~mL})$. The combined organic extract was dried over $\mathrm{MgSO}_{4}$ and then purified by flash chromatography (30\% EtOAc/Hexanes) to give the product as a colorless oil (355 mg, 86\%). IR (neat) 3387, 2934, 2861, 1731, 1300, 972. ${ }^{1} \mathrm{H}$ NMR (300 MHz, $\left.\mathrm{CDCl}_{3}\right) \delta 5.70-5.55(\mathrm{~m}, 2 \mathrm{H}), 4.14(\mathrm{q}, J=7.2 \mathrm{~Hz}, 2 \mathrm{H}), 4.04$, (d, $J=3.6 \mathrm{~Hz}, 2 \mathrm{H}), 3.99-3.91(\mathrm{~m}, 1 \mathrm{H}), 3.14(\mathrm{br} \mathrm{s}, 1 \mathrm{H}), 2.46(\mathrm{dd}, J=16.2,3.6 \mathrm{~Hz}, 1 \mathrm{H}), 2.37$ $(\mathrm{dd}, J=16.2,8.4 \mathrm{~Hz}, 1 \mathrm{H}), 2.08-2.01(\mathrm{~m}, 2 \mathrm{H}), 1.95(\mathrm{br} \mathrm{s}, 1 \mathrm{H}), 1.55-1.38(\mathrm{~m}, 4 \mathrm{H}), 1.24(\mathrm{t}$, $J=7.2 \mathrm{~Hz}, 3 \mathrm{H}) .{ }^{13} \mathrm{C} \mathrm{NMR}\left(75 \mathrm{MHz}, \mathrm{CDCl}_{3}\right) \delta 172.9,132.4,129.4,67.8,63.5,60.6,41.3$, $35.8,31.8,24.8$, 14.1. HRMS (ESI) Calcd for $\mathrm{C}_{11} \mathrm{H}_{26} \mathrm{NaO}_{4}(\mathrm{M}+\mathrm{Na})^{+} 239.1254$, found 239.1262 .

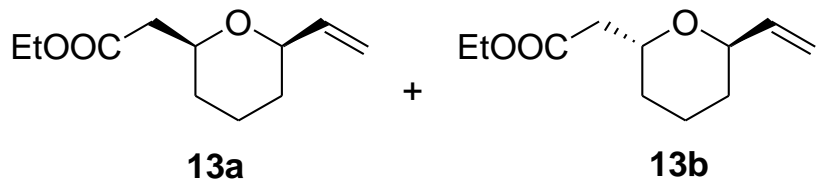

Dry $\mathrm{CH}_{2} \mathrm{Cl}_{2}(0.5 \mathrm{~mL})$ was added to an aluminum foil covered test tube containing $\mathrm{PPh}_{3} \mathrm{AuCl}(1.0 \mathrm{mg}, 0.002 \mathrm{mmol}), \mathrm{AgOTf}(0.5 \mathrm{mg}, 0.002 \mathrm{mmol})$ and activated MS-4 $(17$ $\mathrm{mg})$. After stirring for 10 minutes, cooled to $-10^{\circ} \mathrm{C}$, a solution of diol $12(43.2 \mathrm{mg}, 0.2$ mmol) in dry $\mathrm{CH}_{2} \mathrm{Cl}_{2}(0.5 \mathrm{~mL})$ was added. After TLC analysis showed the reaction to be complete $\left(9 \mathrm{~h}\right.$ ), it was diluted with $\mathrm{CH}_{2} \mathrm{Cl}_{2}$ and filtered through a short plug of silica. The solution of crude product was concentrated, and then purified by flash chromatography (50\% $\mathrm{CH}_{2} \mathrm{Cl}_{2} /$ hexanes) to give the trans and cis products as colorless oil.

Cis-Ethyl 2-(6-vinyl-tetrahydro-2H-pyran-2-yl) acetate (13a). (36.2 mg, $91 \%)$. IR (neat) $2982,2936,2860,1737,1647,1192 .{ }^{1} \mathrm{H}$ NMR $\left(300 \mathrm{MHz}, \mathrm{CDCl}_{3}\right) \delta 5.82$ (ddd, $J=$ $17.4,10.5,4.8 \mathrm{~Hz}, 1 \mathrm{H}), 5.19(\mathrm{dt}, J=17.4,1.5 \mathrm{~Hz}, 1 \mathrm{H}), 5.05(\mathrm{dt}, J=10.5,1.5 \mathrm{~Hz}, 1 \mathrm{H})$, $4.13(\mathrm{q}, J=7.2 \mathrm{~Hz}, 2 \mathrm{H}), 3.87-3.77(\mathrm{~m}, 2 \mathrm{H}), 2.58(\mathrm{dd}, J=15.0,6.9 \mathrm{~Hz}, 1 \mathrm{H}), 2.39(\mathrm{dd}, J=$ 15.0, $6.3 \mathrm{~Hz}, 1 \mathrm{H}), 1.87-1.81(\mathrm{~m}, 1 \mathrm{H}), 1.70-1.55(\mathrm{~m}, 3 \mathrm{H}), 1.30-1.21(\mathrm{~m}, 2 \mathrm{H}), 1.24(\mathrm{t}, J=$ $7.2 \mathrm{~Hz}, 3 \mathrm{H}) .{ }^{13} \mathrm{C} \mathrm{NMR}\left(75 \mathrm{MHz}, \mathrm{CDCl}_{3}\right) \delta 171.3,139.2,114.4,78.2,74.3,60.3,41.7$, 31.0, 30.9, 23.2, 14.2. HRMS (ESI) Calcd for $\mathrm{C}_{11} \mathrm{H}_{19} \mathrm{O}_{3}(\mathrm{M}+\mathrm{H})^{+}$199.1329, found 199.1328 . 
Trans-Ethyl 2-(6-vinyl-tetrahydro-2H-pyran-2-yl) acetate (13b). (3.1 mg, $8 \%$ ). IR (neat) $2931,2855,1738,1038 .{ }^{1} \mathrm{H}$ NMR $\left(300 \mathrm{MHz}, \mathrm{CDCl}_{3}\right) \delta 5.88$ (ddd, $J=17.7,11.1$, $4.2 \mathrm{~Hz}, 1 \mathrm{H}), 5.25$ (dt, $J=17.7,1.8 \mathrm{~Hz}, 1 \mathrm{H}), 5.20$ (dt, $J=11.1,1.8 \mathrm{~Hz}, 1 \mathrm{H}), 4.41-4.36$ (m, $1 \mathrm{H}), 4.25-4.17(\mathrm{~m}, 1 \mathrm{H}), 4.14(\mathrm{q}, J=7.2 \mathrm{~Hz}, 2 \mathrm{H}), 2.58(\mathrm{dd}, J=14.7,8.4 \mathrm{~Hz}, 1 \mathrm{H}), 2.39$ (dd, $J=14.7,5.4 \mathrm{~Hz}, 1 \mathrm{H}), 1.79-1.61(\mathrm{~m}, 4 \mathrm{H}), 1.41-1.23(\mathrm{~m}, 2 \mathrm{H}), 1.26(\mathrm{t}, J=7.2 \mathrm{~Hz}, 3 \mathrm{H})$. ${ }^{13} \mathrm{C}$ NMR $\left(75 \mathrm{MHz}, \mathrm{CDCl}_{3}\right) \delta 171.4,138.3,116.1,72.5,68.0,60.4,40.4,30.4,28.6,18.6$, 14.2. HRMS (ESI) Calcd for $\mathrm{C}_{11} \mathrm{H}_{19} \mathrm{O}_{3}(\mathrm{M}+\mathrm{H})^{+}$199.1329, found 199.1339.

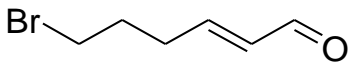

(E)-6-bromohex-2-enal (14a). To a solution of Grubb's catalyst $2^{\text {nd }}$ generation $(1 \mathrm{~mol} \%$, $84.9 \mathrm{mg})$ in dry $\mathrm{CH}_{2} \mathrm{Cl}_{2}(40 \mathrm{~mL})$ in a flamed dried flask was added solution of 5-bromopent-1-ene $(1.49 \mathrm{~g}, 10 \mathrm{mmol})$ and crotonaldehyde $(3.5 \mathrm{~g}, 50 \mathrm{mmol})$ in dry $\mathrm{CH}_{2} \mathrm{Cl}_{2}(10 \mathrm{~mL})$. The mixture was stirred at reflux for 20 hours, cooled to r.t. and then filtered through a short plug of silica. The solvent was removed and the crude was purified by flash chromatography (20\% Ether/Hexanes) to give the product as a colorless oil $(1.69 \mathrm{~g}, 96 \%) .{ }^{1} \mathrm{H}$ NMR $\left(300 \mathrm{MHz}, \mathrm{CDCl}_{3}\right) \delta 9.51(\mathrm{~d}, J=7.2 \mathrm{~Hz}, 1 \mathrm{H}), 6.82(\mathrm{dt}, J=$ 15.6, 7.2 Hz, 1H), 6.15 (ddt, $J=15.6,8.1,1.8 \mathrm{~Hz}, 1 \mathrm{H}), 3.43$ (t, $J=6.6 \mathrm{~Hz}, 2 \mathrm{H}), 2.51$ (dq, $J=6.9,1.5 \mathrm{~Hz}, 2 \mathrm{H}), 2.06(\mathrm{dt}, J=14.4,7.2 \mathrm{~Hz}, 2 \mathrm{H}) .{ }^{13} \mathrm{C} \mathrm{NMR}\left(75 \mathrm{MHz}, \mathrm{CDCl}_{3}\right) \delta 193.7$, $156.0,133.7,32.3,30.9,30.5$.

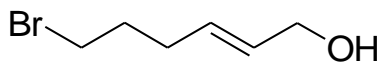

(E)-6-bromohex-2-en-1-ol (14b). To a solution of (E)-6-bromohex-2-enal 14a (266 mg, $1.5 \mathrm{mmol})$ in THF $(6 \mathrm{~mL})$ at $-78^{\circ} \mathrm{C}$ was added DIBAL-H $(1.6 \mathrm{~mL}, 1.0 \mathrm{M}$ in hexane $)$ over 2 min. After stirring at $-78^{\circ} \mathrm{C}$ for $30 \mathrm{~min}$, a saturated aqueous solution of $\mathrm{NH}_{4} \mathrm{Cl}(3 \mathrm{~mL})$ was added, followed by $5 \mathrm{~mL}$ water and $1 \mathrm{~mL} 6 \mathrm{~N} \mathrm{HCl}$. The aqueous layer was extracted with ether $(3 \times 8 \mathrm{~mL})$. The combined extract was dried over $\mathrm{MgSO}_{4}$ and then purified by flash chromatography (50\% Ether/Hexanes) to give the product as a colorless oil (253 mg, $95 \%$ ) that satisfactorily matched all previously reported data. ${ }^{6}$

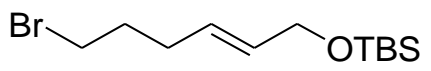

(E)-(6-bromohex-2-enyloxy)(tert-butyl)dimethylsilane (14c). To a solution of (E)-6-bromohex-2-en-1-ol 14b (859 mg, $4.8 \mathrm{mmol})$ and imidazole $(544 \mathrm{mg}, 8.0 \mathrm{mmol})$ in dry $\mathrm{CH}_{2} \mathrm{Cl}_{2}(15 \mathrm{~mL})$ at $0^{\circ} \mathrm{C}$ was added portion-wise TBDMSCl (600 mg, $\left.4.0 \mathrm{mmol}\right)$. The reaction was stirred at r.t. for $1.5 \mathrm{~h}, 10 \mathrm{~mL}$ water was added, and then $1 \mathrm{M} \mathrm{HCl}(4 \mathrm{~mL})$. The aqueous layer was extracted with $\mathrm{CH}_{2} \mathrm{Cl}_{2}(3 \times 10 \mathrm{~mL})$. The combined extracts were dried over $\mathrm{MgSO}_{4}$ and then purified by flash chromatography (20\% Ether/Hexanes) to give the product as a colorless oil (905 mg, 77\%). ${ }^{1} \mathrm{H}$ NMR $\left(300 \mathrm{MHz}, \mathrm{CDCl}_{3}\right) \delta$ 5.63-5.59 (m, 2H), 4.13-4.11 (m, 2H), $3.41(\mathrm{t}, J=6.6 \mathrm{~Hz}, 2 \mathrm{H}), 2.23-2.16(\mathrm{~m}, 2 \mathrm{H})$, 
1.98-1.89 (m, 2H), $0.91(\mathrm{~s}, 9 \mathrm{H}), 0.07(\mathrm{~s}, 6 \mathrm{H}) .{ }^{13} \mathrm{C} \mathrm{NMR}\left(75 \mathrm{MHz}, \mathrm{CDCl}_{3}\right) \delta 130.8,128.7$, 63.7, 33.2, 32.1, 30.5, 25.9, 18.4, -5.1.

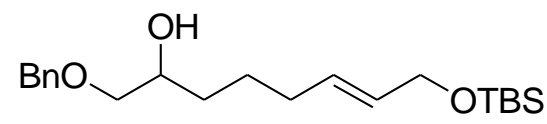

(E)-1-(benzyloxy)-8-(tert-butyldimethylsilyloxy)oct-6-en-2-ol (14d). To a solution of $t$-BuLi $\left(1.2 \mathrm{~mL}, 1.7 \mathrm{M}\right.$ in pentane) in ether $(0.5 \mathrm{~mL})$ at $-78^{\circ} \mathrm{C}$ was added a solution of (E)-(6-bromohex-2-enyloxy)(tert-butyl)dimethylsilane $\mathbf{1 4 c}$ in $0.5 \mathrm{~mL}$ ether. After stirring for $0.5 \mathrm{~h}$ at $-78^{\circ} \mathrm{C}$, a mixture of 2-(benzyloxy)acetaldehyde $(150 \mathrm{mg}, 1.0 \mathrm{mmol})$ and dry ether $(1.0 \mathrm{~mL})$ was added. After the mixture was stirred for 45 minutes, saturated $\mathrm{NH}_{4} \mathrm{Cl}$ solution $(2 \mathrm{~mL})$ and water $(3 \mathrm{~mL})$ was added and the aqueous layer was extracted with ether $(3 \times 10 \mathrm{~mL})$, the combined extracts were dried over $\mathrm{MgSO}_{4}$, and evaporated in vacuum. The residue was subjected to flash chromatography to furnish the product as a colorless oil (117 mg, 32\%). ${ }^{1} \mathrm{H}$ NMR $\left(300 \mathrm{MHz}, \mathrm{CDCl}_{3}\right) \delta$ 7.39-7.26 (m, 5H), 5.68-5.48 (m, 2H), $4.55(\mathrm{~s}, 2 \mathrm{H}), 4.11(\mathrm{dd}, J=5.1,1.2 \mathrm{~Hz}, 2 \mathrm{H}), 3.83-3.78(\mathrm{~m}, 1 \mathrm{H}), 3.50$ (dd, $J=9.3$, $3.0 \mathrm{~Hz}, 1 \mathrm{H}$ ), 3.32 (dd, $J=9.3,7.8 \mathrm{~Hz}, 1 \mathrm{H}), 2.37$ (br s, $1 \mathrm{H}), 2.09-2.02(\mathrm{~m}, 2 \mathrm{H}), 1.56-1.41$ (m, 4H), $0.91(\mathrm{~s}, 9 \mathrm{H}), 0.07$ (s, 6H). ${ }^{13} \mathrm{C}$ NMR $\left(75 \mathrm{MHz}, \mathrm{CDCl}_{3}\right) \delta 137.9,130.8,129.6$, $128.4,127.8,127.7,74.6,73.3,70.2,64.0,32.6,32.1,26.0,25.0,18.4,-5.1$.

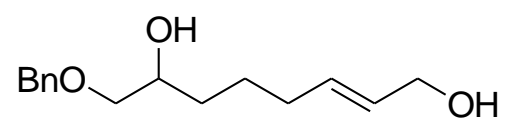

(E)-8-(benzyloxy)oct-2-ene-1,7-diol (14). $p$ - Toluenesulfonic acid hydrate $(13.9 \mathrm{mg}$, $0.073 \mathrm{mmol}$ ) was added to a solution of (E)-1-(benzyloxy)-8-(tert-butyldimethylsilyloxy) oct-6-en-2-ol 14d (267 mg, $0.73 \mathrm{mmol})$ in dry methanol $(7.3 \mathrm{~mL})$. After $20 \mathrm{~min}$, analysis of the reaction by TLC indicated that the starting material had been consumed. The reaction mixture was then diluted with ether $(30 \mathrm{~mL})$ and washed sequentially with saturated aqueous $\mathrm{NaHCO}_{3}(10 \mathrm{~mL})$ and brine $(2 \times 10 \mathrm{~mL})$. The organic layer was dried over $\mathrm{MgSO}_{4}$ and the solvent removed in vacuo. Flash chromatography (40\% Ethyl acetate/Hexane) afforded the product as a colorless oil (150 mg, 82\%). IR (neat) 3384, 2929, 2860, 1496, 1090, 735. ${ }^{1} \mathrm{H}$ NMR $\left(300 \mathrm{MHz}, \mathrm{CDCl}_{3}\right) \delta$ 7.39-7.26 (m, 5H), $5.73-5.58(\mathrm{~m}, 2 \mathrm{H}), 4.55(\mathrm{~s}, 2 \mathrm{H}), 4.07(\mathrm{~d}, J=3.6 \mathrm{~Hz}, 2 \mathrm{H}), 3.82-3.78(\mathrm{~m}, 1 \mathrm{H}), 3.50(\mathrm{dd}, J=$ 9.3, 3.3 Hz, 1H), 3.32 (dd, $J=9.3,8.1 \mathrm{~Hz}, 1 \mathrm{H}), 2.37$ (br s, 1H), 2.10-2.04 (m, 2H), 1.63-1.25 (m, 5H). ${ }^{13} \mathrm{C}$ NMR (75 MHz, $\left.\mathrm{CDCl}_{3}\right) \delta 137.9,132.7,129.3,128.4,127.8,127.7$, 74.6, 73.3, 70.2, 63.7, 32.5, 32.1, 25.0. HRMS (ESI) Calcd for $\mathrm{C}_{15} \mathrm{H}_{21} \mathrm{O}_{2}\left(\mathrm{M}+\mathrm{H}-\mathrm{H}{ }_{2} \mathrm{O}\right)^{+}$ 233.1536, found 233.1537 .

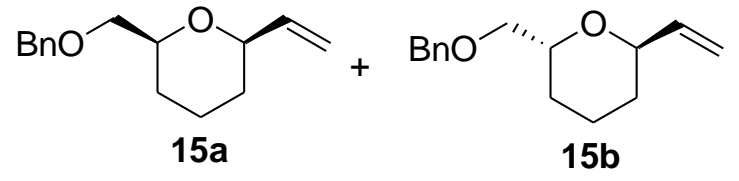


Dry $\mathrm{CH}_{2} \mathrm{Cl}_{2}(0.5 \mathrm{~mL})$ was added to an aluminum foil covered test tube containing

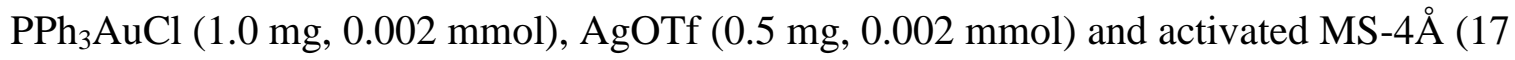
$\mathrm{mg})$. After stirring for 10 minutes, cooled to $-50^{\circ} \mathrm{C}$, a solution of diol $14(50.0 \mathrm{mg}, 0.2$ mmol) in dry $\mathrm{CH}_{2} \mathrm{Cl}_{2}(0.5 \mathrm{~mL})$ was added. After TLC analysis showed the reaction to be complete $\left(24 \mathrm{~h}\right.$ ), it was diluted with $\mathrm{CH}_{2} \mathrm{Cl}_{2}$ and filtered through a short plug of silica. The solution of crude product was concentrated, and then purified by flash chromatography $\left(50 \% \mathrm{CH}_{2} \mathrm{Cl}_{2} /\right.$ hexanes) to give the trans and cis products as colorless oil.

Cis-2-(benzyloxymethyl)-6-vinyl-tetrahydro-2H-pyran (15a). (39.8 mg, 86\%) IR (neat) 2934, 2857, 1496, 1089, 735, 698. ${ }^{1} \mathrm{H}$ NMR $\left(300 \mathrm{MHz}, \mathrm{CDCl}_{3}\right) \delta$ 7.35-7.25 (m, 5H), 5.89 (ddd, $J=17.4,10.5,5.7 \mathrm{~Hz}, 1 \mathrm{H}), 5.23(\mathrm{dt}, J=17.4,1.5 \mathrm{~Hz}, 1 \mathrm{H}), 5.09(\mathrm{dt}, J=10.5$, $1.5 \mathrm{~Hz}, 1 \mathrm{H}), 4.58(\mathrm{dd}, J=16.8,12.6 \mathrm{~Hz}, 2 \mathrm{H}), 3.90-3.84(\mathrm{~m}, 1 \mathrm{H}), 3.67-3.59(\mathrm{~m}, 1 \mathrm{H})$, $3.54(\mathrm{dd}, J=9.9,5.7 \mathrm{~Hz}, 1 \mathrm{H}), 3.43(\mathrm{dd}, J=9.9,4.5 \mathrm{~Hz}, 1 \mathrm{H}), 1.93-1.84(\mathrm{~m}, 1 \mathrm{H})$, $1.67-1.50(\mathrm{~m}, 3 \mathrm{H}), 1.40-1.25(\mathrm{~m}, 2 \mathrm{H}) .{ }^{13} \mathrm{C}$ NMR $\left(75 \mathrm{MHz}, \mathrm{CDCl}_{3}\right) \delta 139.4,138.4,128.3$, 127.7, 127.5, 114.6, 78.3, 76.8, 73.6, 73.4, 31.3, 28.0, 23.1. HRMS (ESI) Calcd for $\mathrm{C}_{15} \mathrm{H}_{19} \mathrm{O}_{2}(\mathrm{M}-\mathrm{H})^{+} 231.1385$, found 231.1374 .

The relative configuration of the major diastereomer was determined by NOE DIFF experiments as follows:

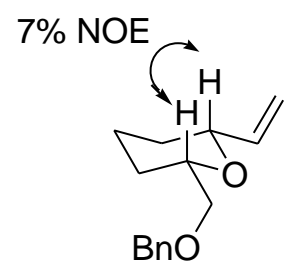

Trans-2-(benzyloxymethyl)-6-vinyl-tetrahydro-2 $\boldsymbol{H}$-pyran (15b). (5.2 $\mathrm{mg}, 11 \%)$ IR (neat) $2933,2858,1454,1102,735,698 .{ }^{1} \mathrm{H}$ NMR $\left(300 \mathrm{MHz}, \mathrm{CDCl}_{3}\right) \delta$ 7.35-7.26 (m, $5 \mathrm{H}), 5.95(\mathrm{ddd}, J=17.7,11.1,4.5 \mathrm{~Hz}, 1 \mathrm{H}), 5.24(\mathrm{dt}, J=17.7,1.8 \mathrm{~Hz}, 1 \mathrm{H}), 5.21(\mathrm{dt}, J=$ $11.1,1.8 \mathrm{~Hz}, 1 \mathrm{H}), 4.57(\mathrm{dd}, J=15.3,12.3 \mathrm{~Hz}, 2 \mathrm{H}), 4.42-4.40(\mathrm{~m}, 1 \mathrm{H}), 3.99-3.90(\mathrm{~m}, 1 \mathrm{H})$, $3.54(\mathrm{dd}, J=10.2,6.0 \mathrm{~Hz}, 1 \mathrm{H}), 3.44(\mathrm{dd}, J=10.2,5.1 \mathrm{~Hz}, 1 \mathrm{H}), 1.82-1.37(\mathrm{~m}, 6 \mathrm{H}) .{ }^{13} \mathrm{C}$ NMR $\left(75 \mathrm{MHz}, \mathrm{CDCl}_{3}\right) \delta 138.43,138.42,128.3,127.6,127.5,116.1,73.3,72.8,72.6$, 70.1, 28.7, 27.6, 18.5. HRMS (ESI) Calcd for $\mathrm{C}_{15} \mathrm{H}_{19} \mathrm{O}_{2}(\mathrm{M}-\mathrm{H})^{+}$231.1385, found 231.1381 .

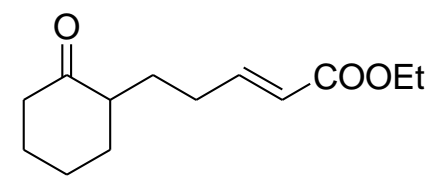

Ethyl 5-(2-oxocyclohexyl) pent-2-enoate (16a). A solution of 1-N-pyrrolidylcyclohexene $(4.8 \mathrm{~mL}, 30 \mathrm{mmol})$ in anhydrous ether $(23 \mathrm{~mL})$ was cooled to $-7^{\circ} \mathrm{C}$. With efficient stirring and cooling in an ice-salt bath, acrolein $(1.71 \mathrm{~g}, 30.5 \mathrm{mmol})$ 
in $2 \mathrm{~mL}$ of ether was added over $100 \mathrm{~min}$. The temperature was kept between -7 and $-2^{\circ} \mathrm{C}$

during the addition, and then was kept at $0^{\circ} \mathrm{C}$ for another hour, when $1.0 \mathrm{~mL}$ of water was added. After the solution was stirred for 20 min., $4.9 \mathrm{~mL}$ of $6 \mathrm{~N}$ hydrochloric acid was added to bring the $\mathrm{pH}$ to 5-6. The ether layer was separated, washed with a saturated sodium bicarbonate solution, dried over $\mathrm{MgSO}_{4}$ and concentrated. The crude 3-(2-oxocyclohexyl)propanal was used in next step directly.

To a solution of the above crude aldehyde in $15 \mathrm{~mL}$ of $\mathrm{MeCN}$ at $0^{\circ} \mathrm{C}$ was added Ethyl (triphenylphosphoranyliden)acetate $(3.98 \mathrm{~g}, 11.4 \mathrm{mmol})$, the mixture was stirred at r.t for $5 \mathrm{~h}$. The solvent was removed in vacuo and the solid was repeatedly washed with ether. The etheral layer was concentrated in vacuo, and then purified by flash chromatography (10\% Ethyl acetate/hexanes) to give the $E$ and $Z$ mixture $(E / Z=10 / 1)$ as colorless oil (1.31 g, $20 \%$ for two steps) that satisfactorily matched all previously reported data. ${ }^{7}$<smiles>OC/C=C/CCC1CCCCC1O</smiles>

Trans-(E)-2-(5-hydroxypent-3-enyl) cyclohexanol (16). To a solution of Ethyl 5-(2-oxocyclohexyl) pent-2-enoate $16 \mathbf{a}(1.31 \mathrm{~g}, 5.8 \mathrm{mmol})$ in $\mathrm{THF}(10 \mathrm{~mL})$ at $-78^{\circ} \mathrm{C}$ was added DIBAL-H (18.4 mL, 1.0 M in hexane) over 5 minutes. After stirring at $-78^{\circ} \mathrm{C}$ for 2 $\mathrm{h}$, the reaction mixture was poured into $6 \mathrm{~N} \mathrm{HCl}(10 \mathrm{~mL})$ and the aqueous layer was extracted with ether $(4 \times 10 \mathrm{~mL})$. The combined extract was dried over $\mathrm{MgSO}_{4}$ and then purified by flash chromatography ( $25 \%$ Ether/Hexanes) to give the products as colorless oil. The diols are partially separable, and $298 \mathrm{mg}$ of Trans-(E)-2-(5-hydroxypent-3-enyl) cyclohexanol 16 was obtained. IR (neat) 3331, 2926, 2855, 1670, 1448, 969. ${ }^{1} \mathrm{H}$ NMR $\left(300 \mathrm{MHz}, \mathrm{CDCl}_{3}\right) \delta 5.68-5.52(\mathrm{~m}, 2 \mathrm{H}), 3.99(\mathrm{~d}, J=4.2 \mathrm{~Hz}, 2 \mathrm{H}), 3.17-3.11(\mathrm{~m}, 1 \mathrm{H}), 2.80$ (br s, 2H), 2.16-1.55 (m, 7H), 1.23-0.78 (m, 5H). ${ }^{13} \mathrm{C}$ NMR (75 MHz, $\left.\mathrm{CDCl}_{3}\right) \delta 133.0$, 128.9, 74.3, 63.2, 44.1, 35.6, 31.5, 30.0, 29.0, 25.3, 24.8. HRMS (ESI) Calcd for $\mathrm{C}_{11} \mathrm{H}_{19} \mathrm{O}$ $\left(\mathrm{M}+\mathrm{H}-\mathrm{H}_{2} \mathrm{O}\right)^{+} 167.1430$, found 167.1427 .

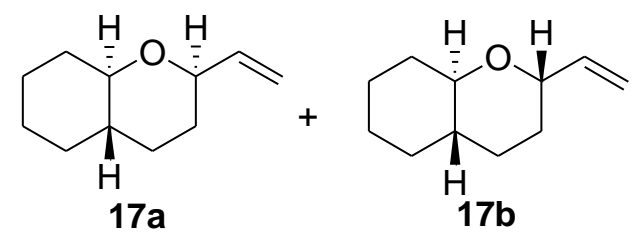

Dry $\mathrm{CH}_{2} \mathrm{Cl}_{2}(0.5 \mathrm{~mL})$ was added to an aluminum foil covered test tube containing $\mathrm{PPh}_{3} \mathrm{AuCl}(5.0 \mathrm{mg}, 0.01 \mathrm{mmol}), \mathrm{AgOTf}(2.5 \mathrm{mg}, 0.01 \mathrm{mmol})$ and activated MS-4A (17 
$\mathrm{mg})$. After stirring for 10 minutes, cooled to $-78^{\circ} \mathrm{C}$, a solution of diol $16(36.8 \mathrm{mg}, 0.2$ mmol) in dry $\mathrm{CH}_{2} \mathrm{Cl}_{2}(0.5 \mathrm{~mL})$ was added. After TLC analysis showed the reaction to be complete $(10 \mathrm{~h})$, it was diluted with $\mathrm{CH}_{2} \mathrm{Cl}_{2}$ and filtered through a short plug of silica. The solution of crude product was concentrated, and then purified by flash chromatography (20\% $\mathrm{CH}_{2} \mathrm{Cl}_{2}$ /hexanes) to give the trans and cis products as colorless oil.

$\left(\mathbf{2} \boldsymbol{R}^{*}, \mathbf{4 a S} \boldsymbol{*}, \mathbf{8 a} \boldsymbol{R} \boldsymbol{*}\right)$-2-vinyl-octahydro-2H-chromene (17a). (23.7 mg, $\left.71.4 \%\right)$ IR (neat) 2925, 2854, 1462, 1377, 1080. ${ }^{1} \mathrm{H}$ NMR $\left(300 \mathrm{MHz}, \mathrm{CDCl}_{3}\right) \delta 5.87$ (ddd, $J=17.4,10.5$, $5.7 \mathrm{~Hz}, 1 \mathrm{H}), 5.23(\mathrm{dt}, J=17.4,1.5 \mathrm{~Hz}, 1 \mathrm{H}), 5.08$ (dt, $J=10.5,1.5 \mathrm{~Hz}, 1 \mathrm{H}), 3.87-3.81(\mathrm{~m}$, $1 \mathrm{H}), 3.02-2.94(\mathrm{~m}, 1 \mathrm{H}), 1.94-1.01(\mathrm{~m}, 13 \mathrm{H}) .{ }^{13} \mathrm{C} \mathrm{NMR}\left(75 \mathrm{MHz}, \mathrm{CDCl}_{3}\right) \delta 139.5,114.7$, 81.8, 78.5, 41.5, 32.6, 32.2, 31.7, 30.7, 25.8, 25.1. HRMS (ESI) Calcd for $\mathrm{C}_{11} \mathrm{H}_{17} \mathrm{O}$ $(\mathrm{M}-\mathrm{H})^{+} 165.1274$, found 165.1271 .

The relative configuration of the major diastereomer was determined by NOE DIFF experiments as follows:

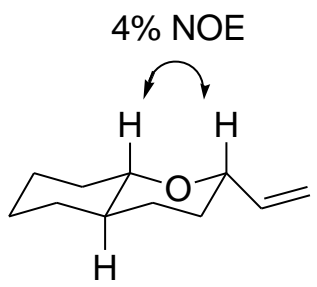

$\left(2 S^{*}, \mathbf{4 a} S^{*}, \mathbf{8 a} R^{*}\right)$-2-vinyl-octahydro-2H-chromene (17b). (2.6 mg, $\left.7.8 \%\right)$ IR (neat) 2930, 2856, 1727, 1448, 1286. ${ }^{1} \mathrm{H}$ NMR $\left(300 \mathrm{MHz}, \mathrm{CDCl}_{3}\right) \delta 6.01$ (ddd, $J=17.4,11.4$, $4.2 \mathrm{~Hz}, 1 \mathrm{H}), 5.24$ (dt, $J=11.4,1.8 \mathrm{~Hz}, 1 \mathrm{H}), 5.21$ (dt, $J=17.4,1.8 \mathrm{~Hz}, 1 \mathrm{H}), 4.50-4.45$ (m, $1 \mathrm{H}), 3.23$ (dt, $J=9.9,3.9 \mathrm{~Hz}, 1 \mathrm{H}), 1.99-1.14$ (m, 13H). HRMS (ESI) Calcd for $\mathrm{C}_{11} \mathrm{H}_{17} \mathrm{O}$ $(\mathrm{M}-\mathrm{H})^{+} 165.1274$, found 165.1264 .

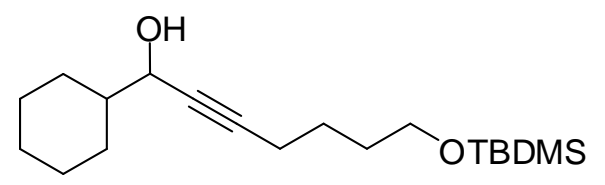

7-(tert-butyldimethylsilyloxy)-1-cyclohexylhept-2-yn-1-ol (18a). A solution of $n$-BuLi in hexane $2.5 \mathrm{M}(1.04 \mathrm{~mL}, 2.6 \mathrm{mmol})$ was added dropwise over 10 minutes at $-78^{\circ} \mathrm{C}$ to a solution of tert-butyl(hex-5-ynyloxy)dimethylsilane ${ }^{8}$ (500.8 $\left.\mathrm{mg}, 2.36 \mathrm{mmol}\right)$ in dry THF $(35 \mathrm{~mL})$. The reaction was then stirred at the same temperature for 45 minutes and a solution of cyclohexane carboxaldehyde $(344.2 \mathrm{mg}, 3.07 \mathrm{mmol})$ in dry THF (3 mL) was added. The mixture was allowed to warm to $-30^{\circ} \mathrm{C}$ and stirred for 30 minutes, quenched with $\mathrm{NH}_{4} \mathrm{Cl}$ (20 mL of a saturated aqueous solution), diluted with water $(20 \mathrm{~mL})$ and extracted with $\mathrm{CH}_{2} \mathrm{Cl}_{2}(2 \times 30 \mathrm{~mL})$. The organic layers were dried over $\mathrm{MgSO}_{4}$ and then purified by flash chromatography (20\% EtOAc/Hexanes) to give the product as a colorless oil (697.2 mg, 91\%). $\mathrm{R}_{\mathrm{f}}=0.42$ (10\% EtOAc/hexanes); IR (neat) 3333, 3011, 
2852, $1446 \mathrm{~cm}^{-1} ;{ }^{1} \mathrm{H}$ NMR $\left(300 \mathrm{MHz}, \mathrm{CDCl}_{3}\right): \delta 4.01(\mathrm{~d}, J=7.2 \mathrm{~Hz}, 1 \mathrm{H}), 3.61(\mathrm{t}, J=7.2$ $\mathrm{Hz}, 2 \mathrm{H}), 2.21(\mathrm{q}, J=7.1 \mathrm{~Hz}, 2 \mathrm{H}), 1.99-0.95(\mathrm{~m}, 15 \mathrm{H}), 0.86(\mathrm{~s}, 9 \mathrm{H}), 2.20-0.91(\mathrm{~m}, 19 \mathrm{H})$, $0.86(\mathrm{~s}, 9 \mathrm{H}), 0.08(\mathrm{~s}, 6 \mathrm{H}) ;{ }^{13} \mathrm{C} \mathrm{NMR}\left(75 \mathrm{MHz}, \mathrm{CDCl}_{3}\right): \delta 86.2,80.5,67.6,62.9,44.5$, 32.1, 28.8, 28.3, 26.6, 26.1, 25.4, 19.7, 18.7, 18.5, -5.1; HRMS (ESI) Calcd for $\mathrm{C}_{19} \mathrm{H}_{35} \mathrm{O}_{2} \mathrm{Si}(\mathrm{M}-\mathrm{H})^{+} 323.2401$, found 323.2398.

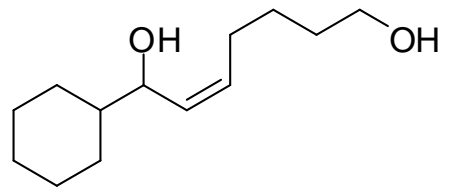

(Z)-1-cyclohexylhept-2-ene-1,7-diol (18). Lindlar catalyst (5\% palladium on calcium carbonate, poisoned with lead, $30 \mathrm{mg}$ ) was added to a solution of 18a $(150.2 \mathrm{mg}, 0.46$ $\mathrm{mmol})$ in a mixture of EtOAc/pyridine/1-hexene $(10: 1: 1,250 \mu \mathrm{L})$. The reaction mixture was stirred 16h under $\mathrm{H}_{2}(1 \mathrm{~atm})$. After filtration over celite and removal of the solvent, crude product was recovered as a colorless oil which was used for the next step without further purification.

A solution of HF pyridine $(550 \mu \mathrm{L})$ was added dropwise at $0^{\circ} \mathrm{C}$ to a solution of the silane obtained above $(116.1 \mathrm{mg}, 0.35 \mathrm{mmol})$ in dry THF $(4 \mathrm{~mL})$. The reaction was stirred for 2 hours at the same temperature and $\mathrm{NaHCO}_{3}$ saturated $(30 \mathrm{~mL}$ of a saturated aqueous solution) was added dropwise. After dilution in water $(20 \mathrm{~mL})$, the crude product was extracted with $\mathrm{CH}_{2} \mathrm{Cl}_{2}(2 \times 30 \mathrm{~mL})$, the combined organic layers were dried over $\mathrm{MgSO}_{4}$ and the solvent removed by vacuum. Flash chromatography (30\% EtOAc/Hexanes) afforded the product as a colorless oil $(58.4 \mathrm{mg}, 78 \%) . \mathrm{R}_{\mathrm{f}}=0.12$ (20\% EtOAc/hexanes); IR (neat) $3330,2924,2852,1449 \mathrm{~cm}^{-1} ;{ }^{1} \mathrm{H}$ NMR $\left(300 \mathrm{MHz}, \mathrm{CDCl}_{3}\right): \delta 5.42(\mathrm{dt}, J=7.2$, $11.1 \mathrm{~Hz}, 1 \mathrm{H}), 5.36(\mathrm{dd}, J=9,10.5 \mathrm{~Hz}, 1 \mathrm{H}), 4.09(\mathrm{t}, J=7.2 \mathrm{~Hz}, 1 \mathrm{H}), 3.61(\mathrm{t}, J=6.3 \mathrm{~Hz}$, $2 \mathrm{H}), 2.06(\mathrm{q}, J=6.9 \mathrm{~Hz}, 2 \mathrm{H}), 2.20-0.81(\mathrm{~m}, 19 \mathrm{H}) ;{ }^{13} \mathrm{C} \mathrm{NMR}\left(75 \mathrm{MHz}, \mathrm{CDCl}_{3}\right): \delta 132.7$, 131.6, 72.1, 62.9, 44.2, 32.4, 29.0, 28.8, 27.7, 26.7, 26.3, 26.2, 26.1; HRMS (ESI) Calcd for $\mathrm{C}_{13} \mathrm{H}_{23} \mathrm{O}_{2}(\mathrm{M}-\mathrm{H})^{+}$211.1693, found 211.1704.

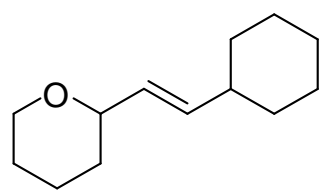

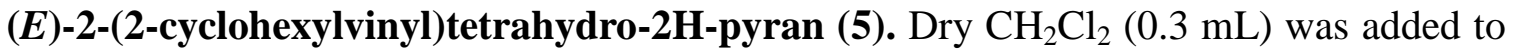
an aluminum foil covered test tube containing $\mathrm{PPh}_{3} \mathrm{AuCl}(0.7 \mathrm{mg}, 0.001 \mathrm{mmol}), \mathrm{AgOTf}$

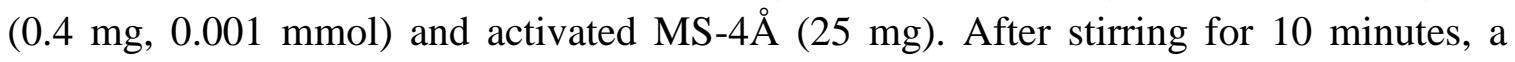
solution of diol $18(21.1 \mathrm{mg}, 0.10 \mathrm{mmol})$ in dry $\mathrm{CH}_{2} \mathrm{Cl}_{2}(0.3 \mathrm{~mL})$ was added. After TLC analysis showed the reaction to be complete (40 min), it was diluted with $\mathrm{CH}_{2} \mathrm{Cl}_{2}$ and filtered through a short plug of silica. The solution of crude product was concentrated, and then purified by flash chromatography ( $5 \%$ EtOAc/hexanes) to give the product as a 
colorless oil (17.9 mg, 92\%) that satisfactorily matched all reported data above.

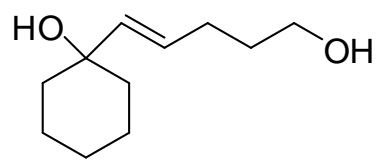

(E)-1-(6-hydroxyhex-1-enyl)-cyclohexanol (19): A solution of hex-5-en-1-ol (100.2 mg, $1 \mathrm{mmol})$ and 1-vinylcyclohexanol $(252.4 \mathrm{mg}, 2 \mathrm{mmol})$ in dry $\mathrm{CH}_{2} \mathrm{Cl}_{2}(2 \mathrm{~mL})$ was added to a solution of Grubb's $2^{\text {nd }}$ generation catalyst $(42.5 \mathrm{mg}, 0.05 \mathrm{mmol}, 5 \mathrm{~mol} \%)$ in dry $\mathrm{CH}_{2} \mathrm{Cl}_{2}(3 \mathrm{~mL})$. The mixture was stirred at reflux for 1 hour and then cooled to rt. Silica gel (200 mg) was added and the reaction mixture was stirred open to air for 1 hour. The solvent was removed and the crude product was purified by flash chromatography (20\% EtOAc/Hexanes) to give the product as a yellow oil (116.8 $\mathrm{mg}, 93 \%) . \mathrm{R}_{\mathrm{f}}=0.18(20 \%$ EtOAc/hexanes); IR (neat) 3417, 2976, 2932, 2860, 1382, 1120, $423 \mathrm{~cm}^{-1} ;{ }^{1} \mathrm{H}$ NMR (300 $\left.\mathrm{MHz}, \mathrm{CDCl}_{3}\right): \delta 5.63(\mathrm{~m}, 2 \mathrm{H}), 3.63(\mathrm{t}, J=6.6 \mathrm{~Hz}, 2 \mathrm{H}), 2.06(\mathrm{q}, J=6.9 \mathrm{~Hz}, 1 \mathrm{H})$, 1.74-1.26 (m, 16H); $\left.{ }^{13} \mathrm{C} \mathrm{NMR} \mathrm{(75} \mathrm{MHz,} \mathrm{CDCl}_{3}\right): \delta 138.2,127.8,89.1,81.9,71.5,62.9$, 38.3, 32.3, 32.2, 25.7, 25.6, 22.4, 22.3; HRMS (ESI) Calcd for $\mathrm{C}_{12} \mathrm{H}_{19} \mathrm{O}_{2}\left(\mathrm{M}-2 \mathrm{H}_{2} \mathrm{O}+\mathrm{H}\right)^{+}$ 163.1487 , found 163.1491 .

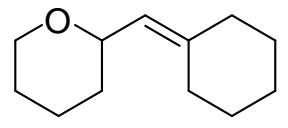

2-(cyclohexylidenemethyl)tetrahydropyran (20). Dry $\mathrm{CH}_{2} \mathrm{Cl}_{2}(0.7 \mathrm{~mL})$ was added to an aluminum foil covered test tube containing $\mathrm{PPh}_{3} \mathrm{AuCl}(1.3 \mathrm{mg}, 0.003 \mathrm{mmol})$, AgOTf

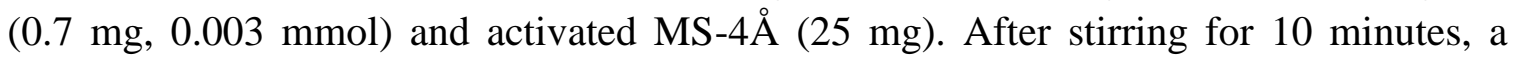
solution of diol $19(51.5 \mathrm{mg}, 0.26 \mathrm{mmol})$ in dry $\mathrm{CH}_{2} \mathrm{Cl}_{2}(0.7 \mathrm{~mL})$ was added. After TLC analysis showed the reaction to be complete $(2.5 \mathrm{~h})$, it was diluted with $\mathrm{CH}_{2} \mathrm{Cl}_{2}$ and filtered through a short plug of silica. The solution of crude product was concentrated, and then purified by flash chromatography (5\% EtOAc/hexanes) to give the product as a colorless oil (42.1 mg, 91\%). $\mathrm{R}_{\mathrm{f}}=0.85$ (5\% EtOAc/hexanes); IR (neat) 2929, 2852, 1086, $1033 \mathrm{~cm}^{-1} ;{ }^{1} \mathrm{H}$ NMR $\left(300 \mathrm{MHz}, \mathrm{CDCl}_{3}\right): \delta 5.08(\mathrm{dd}, J=8.1,0.9 \mathrm{~Hz}, 1 \mathrm{H}), 4.04-3.92(\mathrm{~m}$, $2 \mathrm{H}), 3.44(\mathrm{dt}, J=11.7,2.7 \mathrm{~Hz}, 1 \mathrm{H}), 2.14-2.03(\mathrm{~m}, 4 \mathrm{H}), 1.82-1.22(\mathrm{~m}, 12 \mathrm{H}) ;{ }^{13} \mathrm{C} \mathrm{NMR}$ $\left(75 \mathrm{MHz}, \mathrm{CDCl}_{3}\right): \delta 142.9,123.4,74.4,68.4,37.1,32.8,29.7,28.6,28.0,26.9,26.0,23.7$; HRMS (ESI) Calcd for $\mathrm{C}_{12} \mathrm{H}_{19} \mathrm{O}(\mathrm{M}-\mathrm{H})^{+} 179.1427$, found 179.1436 .

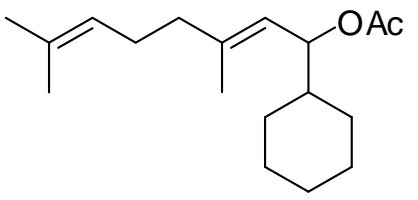

(E)-1-cyclohexyl-3,7-dimethylocta-2,6-dienyl acetate (21a). A solution of cyclohexylmagnesium bromide ( $2 \mathrm{M}$ in $\mathrm{Et}_{2} \mathrm{O}, 1.083 \mathrm{~mL}, 1.1$ eq.) was added dropwise at $0^{\circ} \mathrm{C}$ to a solution of geranial ${ }^{9}(300 \mathrm{mg}, 1.97 \mathrm{mmol})$ in dry THF $(10 \mathrm{~mL})$. The mixture was 
stirred 20 minutes and then acetylchloride $(309 \mu \mathrm{L}, 3.94 \mathrm{mmol})$ was added dropwise. The reaction mixture was warmed to $\mathrm{rt}$ and stirred for $2 \mathrm{~h}$, then quenched with water $(20 \mathrm{~mL})$ and extracted with $\mathrm{Et}_{2} \mathrm{O}(3 \times 20 \mathrm{~mL})$. The organic layers were dried over $\mathrm{MgSO}_{4}$, concentrated, and the crude product was purified by flash chromatography (10\% EtOAc/Hexanes) to give the product as a colorless oil $(477.2 \mathrm{mg}, 87 \%) . \mathrm{R}_{\mathrm{f}}=0.57(20 \%$ EtOAc/hexanes); IR (neat) 2928, 2854, 1727, 1264, 1247, $740 \mathrm{~cm}^{-1} ;{ }^{1} \mathrm{H}$ NMR (300 MHz, $\left.\mathrm{CDCl}_{3}\right): \delta 5.24(\mathrm{dd}, \mathrm{J}=7.5,9.6 \mathrm{~Hz}, 1 \mathrm{H}), 5.00(\mathrm{~m}, 2 \mathrm{H}), 2.07-1.98(\mathrm{~m}, 7 \mathrm{H}), 1.75-0.85(\mathrm{~m}$, $20 \mathrm{H}) ;{ }^{13} \mathrm{C}$ NMR $\left(75 \mathrm{MHz}, \mathrm{CDCl}_{3}\right): \delta 170.7,140.8,131.8,124.2,122.6,75.5,42.4,39.9$, 29.0, 28.5, 26.6, 26.4, 26.2, 26.0, 25.9, 21.5, 17.9, 17.0; HRMS (ESI) Calcd for $\mathrm{C}_{18} \mathrm{H}_{29} \mathrm{O}_{2}$ $(\mathrm{M}-\mathrm{H})^{+}$277.2185, found 277.2168.

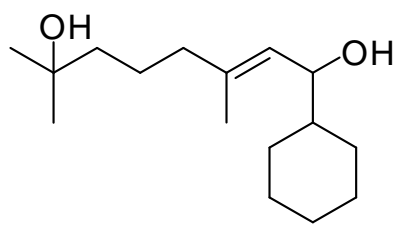

(E)-1-cyclohexyl-3,7-dimethyloct-2-ene-1,7-diol (21). A solution of meta-chloroperoxybenzoic acid (77\% max., $244.7 \mathrm{mg}, 1.09 \mathrm{mmol})$ in $\mathrm{CH}_{2} \mathrm{Cl}_{2}(2 \mathrm{~mL}$ ) was added dropwise at $0^{\circ} \mathrm{C}$ to a solution of $21 \mathrm{a}(276.4 \mathrm{mg}, 0.99 \mathrm{mmol})$ in $\mathrm{CH}_{2} \mathrm{Cl}_{2}(8 \mathrm{~mL})$. After for 3 hours, the reaction mixture was quenched with $\mathrm{NaOH}(10 \mathrm{~mL}$ of a $1 \mathrm{M}$ aqueous solution), diluted with water $(20 \mathrm{~mL})$ and extracted with $\mathrm{CH}_{2} \mathrm{Cl}_{2}(2 \times 40 \mathrm{~mL})$. The combined organic layers were dried over $\mathrm{MgSO}_{4}$ and the solvent removed to give the product as a crude colorless oil which was used for the next step without further purification.

A solution of the epoxide obtained above $(292 \mathrm{mg}, 0.99 \mathrm{mmol})$ in $\mathrm{Et}_{2} \mathrm{O}(5 \mathrm{~mL})$ was added dropwise over $10 \mathrm{~min}$ at $0^{\circ} \mathrm{C}$ to a vigorously stirred suspension of lithium aluminum hydride $95 \%$ (119 mg, $2.98 \mathrm{mmol})$ in dry $\mathrm{Et}_{2} \mathrm{O}(10 \mathrm{~mL})$. The reaction mixture was allowed to warm to $\mathrm{rt}$ and was then stirred at reflux for 45 minutes. The reaction was cooled to $0^{\circ} \mathrm{C}$ and was added successively water $(120 \mu \mathrm{L}), \mathrm{NaOH}(120 \mu \mathrm{L}$ of a $15 \%$ aqueous solution) and then water $(360 \mu \mathrm{L})$. After filtration, the solution was dried over $\mathrm{MgSO}_{4}$ and purified by flash chromatography (15\% to $30 \%$ EtOAc/Hexanes) to give the product as a colorless oil (110.2 mg, 40\% over 2 steps). IR (neat) 3373, 2923, 2851, 1001, $423 \mathrm{~cm}^{-1} 1{ }^{1} \mathrm{H}$ NMR $\left(300 \mathrm{MHz}, \mathrm{CDCl}_{3}\right): \delta 5.15(\mathrm{~d}, J=9.0 \mathrm{~Hz}, 1 \mathrm{H}), 4.04(\mathrm{t}, J=8.7 \mathrm{~Hz}$, $1 \mathrm{H}), 1.99(\mathrm{t}, J=6.6 \mathrm{~Hz}, 2 \mathrm{H}) 1.91-0.86(\mathrm{~m}, 24 \mathrm{H}) .{ }^{13} \mathrm{C} \mathrm{NMR}\left(100 \mathrm{MHz}, \mathrm{CDCl}_{3}\right): \delta 139.05$, $126.84,73.08,71.11,44.52,44.39,43.67,40.27,29.49,29.17,28.79,26.79,26.35,26.23$, 22.63, 16.89. HRMS (ESI) Calcd for $\mathrm{C}_{16} \mathrm{H}_{29} \mathrm{O}_{2}(\mathrm{M}-\mathrm{H})^{+}$253.2162, found 253.2175.

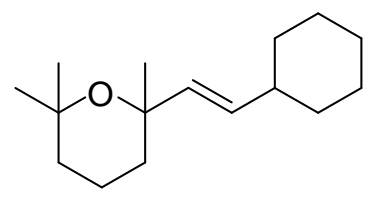

(E)-2-(2-cyclohexylvinyl)-2,6,6-trimethyltetrahydropyran (22). Dry $\mathrm{CH}_{2} \mathrm{Cl}_{2}(0.5$ 
$\mathrm{mL}$ ) was added to an aluminum foil covered test tube containing $\mathrm{PPh}_{3} \mathrm{AuCl}(0.9 \mathrm{mg}$, $0.002 \mathrm{mmol}), \operatorname{AgOTf}(0.5 \mathrm{mg}, 0.002 \mathrm{mmol})$ and activated MS-4̊ $(25 \mathrm{mg})$. After stirring for 10 minutes, a solution of diol $21(45.0 \mathrm{mg}, 0.18 \mathrm{mmol})$ in dry $\mathrm{CH}_{2} \mathrm{Cl}_{2}(0.4 \mathrm{~mL})$ was added. After TLC analysis showed the reaction to be complete (6h), it was diluted with $\mathrm{CH}_{2} \mathrm{Cl}_{2}$ and filtered through a short plug of silica. The solution of crude product was concentrated, and then purified by flash chromatography (5\% EtOAc/hexanes) to give the product as a colorless oil (37.2 $\mathrm{mg}, 89 \%) . \mathrm{R}_{\mathrm{f}}=0.81$ (5\% EtOAc/hexanes); IR (neat) 2970, 2925, 2852, 1093, 430, $409 \mathrm{~cm}^{-1} ;{ }^{1} \mathrm{H}$ NMR (300 MHz, $\left.\mathrm{CDCl}_{3}\right): \delta 5.45$ (d, $J=16.2 \mathrm{~Hz}$, $1 \mathrm{H}), 5.27(\mathrm{dd}, J=15.9,6.3 \mathrm{~Hz}, 1 \mathrm{H}), 1.92-0.81(\mathrm{~m}, 26 \mathrm{H}) .{ }^{13} \mathrm{C} \mathrm{NMR}\left(100 \mathrm{MHz}, \mathrm{CDCl}_{3}\right): \delta$ 136.1, 132.0, 73.3, 72.3, 40.7, 37.0, 33.9, 33.1, 32.9, 32.8, 32.5, 27.7, 26.5, 26.3, 17.2; HRMS (ESI) Calcd for $\mathrm{C}_{16} \mathrm{H}_{27} \mathrm{O}(\mathrm{M}-\mathrm{H})^{+} 235.2049$, found 235.2062.

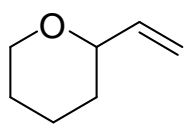

2-vinyltetrahydro-2H-pyran (23a). Dry $\mathrm{CH}_{2} \mathrm{Cl}_{2}(1.0 \mathrm{~mL})$ was added to an aluminum foil covered test tube containing $\mathrm{PPh}_{3} \mathrm{AuCl}(12.0 \mathrm{mg}, 0.025 \mathrm{mmol})$, AgOTf $(6.1 \mathrm{mg}$,

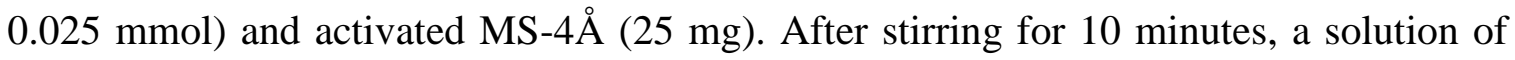
(E)-hex-1-ene-1,6-diol $\mathbf{2 3}^{10}$ (75.2 $\left.\mathrm{mg}, 0.50 \mathrm{mmol}\right)$ in dry $\mathrm{CH}_{2} \mathrm{Cl}_{2}(1.0 \mathrm{~mL})$ was added. After TLC analysis showed the reaction to be complete $(15 \mathrm{~min})$, it was diluted with $\mathrm{CH}_{2} \mathrm{Cl}_{2}$ and filtered through a short plug of silica. The solution of crude product was concentrated, and then purified by flash chromatography (5\% EtOAc/hexanes) to give the product as a colorless oil that satisfactorily matched previously reported data. ${ }^{11}$

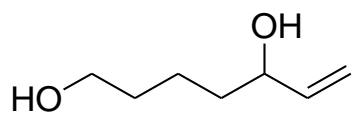

Hept-6-ene-1,5-diol (24). DIBAL-H (22 mL, $1 \mathrm{M}$ in hexane) was added dropwise to a stirred and cooled $\left(-78{ }^{\circ} \mathrm{C}\right)$ solution of $\delta$-valerolactone $(2.0 \mathrm{~g}, 20 \mathrm{mmol})$ in a mixture of pentane-ether $(34 \mathrm{~mL}, 1: 1)$. After $2 \mathrm{~h}$ at $-78{ }^{\circ} \mathrm{C}$ vinylmagnesium chloride $(22.0 \mathrm{~mL}, 1 \mathrm{M}$ in THF) was added. The solution was warmed to $\mathrm{rt}$ and stirred overnight. Ammonium chloride solution was added $(35 \mathrm{~mL})$, and after separation, the aqueous phase was extracted with $\mathrm{CH}_{2} \mathrm{Cl}_{2}(4 \times 30 \mathrm{~mL})$. The combined organic extract was dried $\left(\mathrm{Na}_{2} \mathrm{SO}_{4}\right)$ and concentrated. Chromatography of the residue over silica gel (ether) gave $0.87 \mathrm{~g}$ of diol $(33 \%)$ that satisfactorily matched all previously reported data. ${ }^{12}$

\section{References:}

1. Morgan, J. P.; Grubbs, R. H. Org. Lett. 2000, 2, 3150.

2. Jang, Y.-J.; Shih, Y.-K.; Liu, J.-Y.; Kuo, W.-Y.; Yao, C.-F. Chem. Eur. J. 2003, 9, 2123.

3. (a) Hon, Y.-S.; Liu, Y.-W.; Hsieh, C.-H. Tetrahedron 2004, 60, 4837. (b) Gallagher, T. 


\section{J. Chem. Soc., Chem. Commun. 1984, 1554.}

4. Park, P. K.; O’Malley, S. J.; Schmidt, D. R.; Leighton, J. L. J. Am. Chem. Soc. 2006, $128,2796$.

5. Marotta, E.; Foresti, E.; Marcelli, T.; Peri, F.; Righi, P.; Scardovi, N.; Rosini, G. Org. Lett. 2002, 4, 4451.

6. Fraunhoffer, K. J.; Bachovchin, D. A.; White, M. C. Org. Lett. 2005, 7, 223.

7. Pandey G.; Hajra, S.; Ghorai M. K. J. Org. Chem. 1997, 62, 5966.

8. Molander, G. A.; Fumagalli, T. J. Org. Chem. 2006, 71, 6743

9. Zakharova, S.; Fulhorst, M.; Łuczak, L.; Wessjohann, L. ARKIVOC 2004, 13, 79.

10. Chen, S. H.; Hong, B. C.; Su, C.; Sarshar, S. Tetrahedron Lett. 2005, 46, 8899.

11. Zhang, Z.; Liu, C.; Kinder, R. E.; Han, X.; Qian, H.; Widenhoefer, R. A. J. Am. Chem.. Soc. 2006, 128, 9066.

12. Brunel, Y.; Rousseau, G. J. Org. Chem. 1996, 61, 5793. 


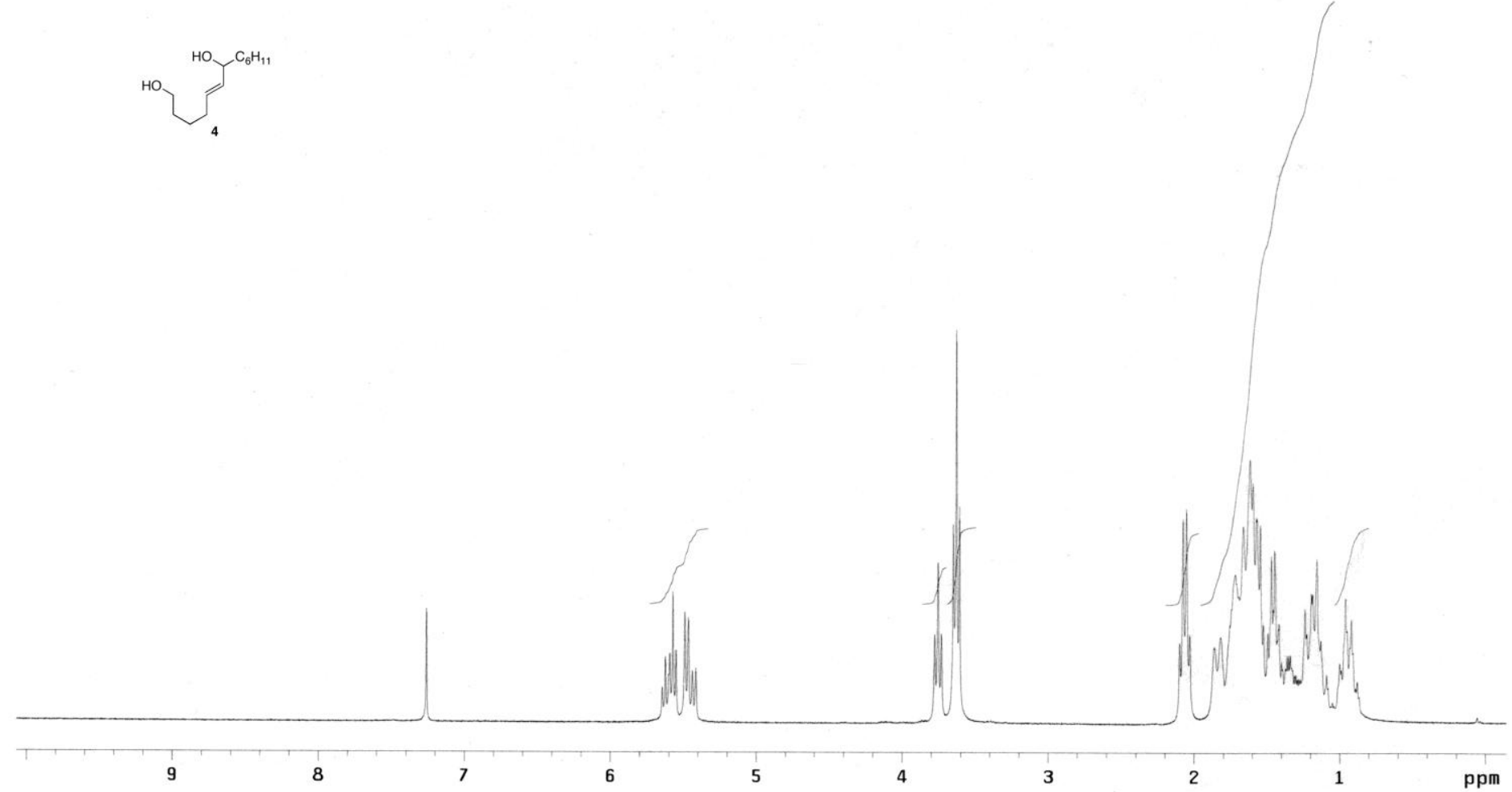

S19 


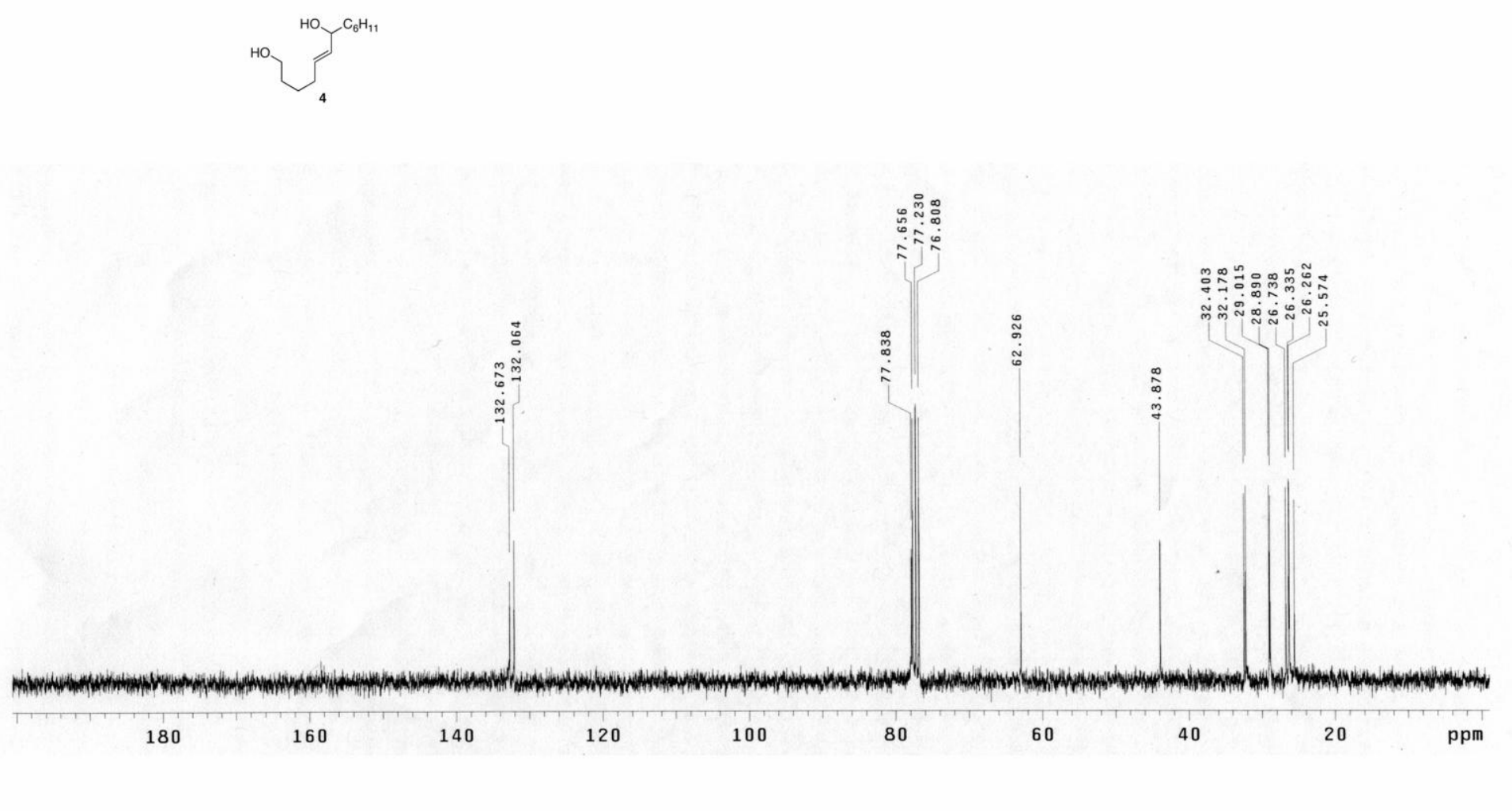



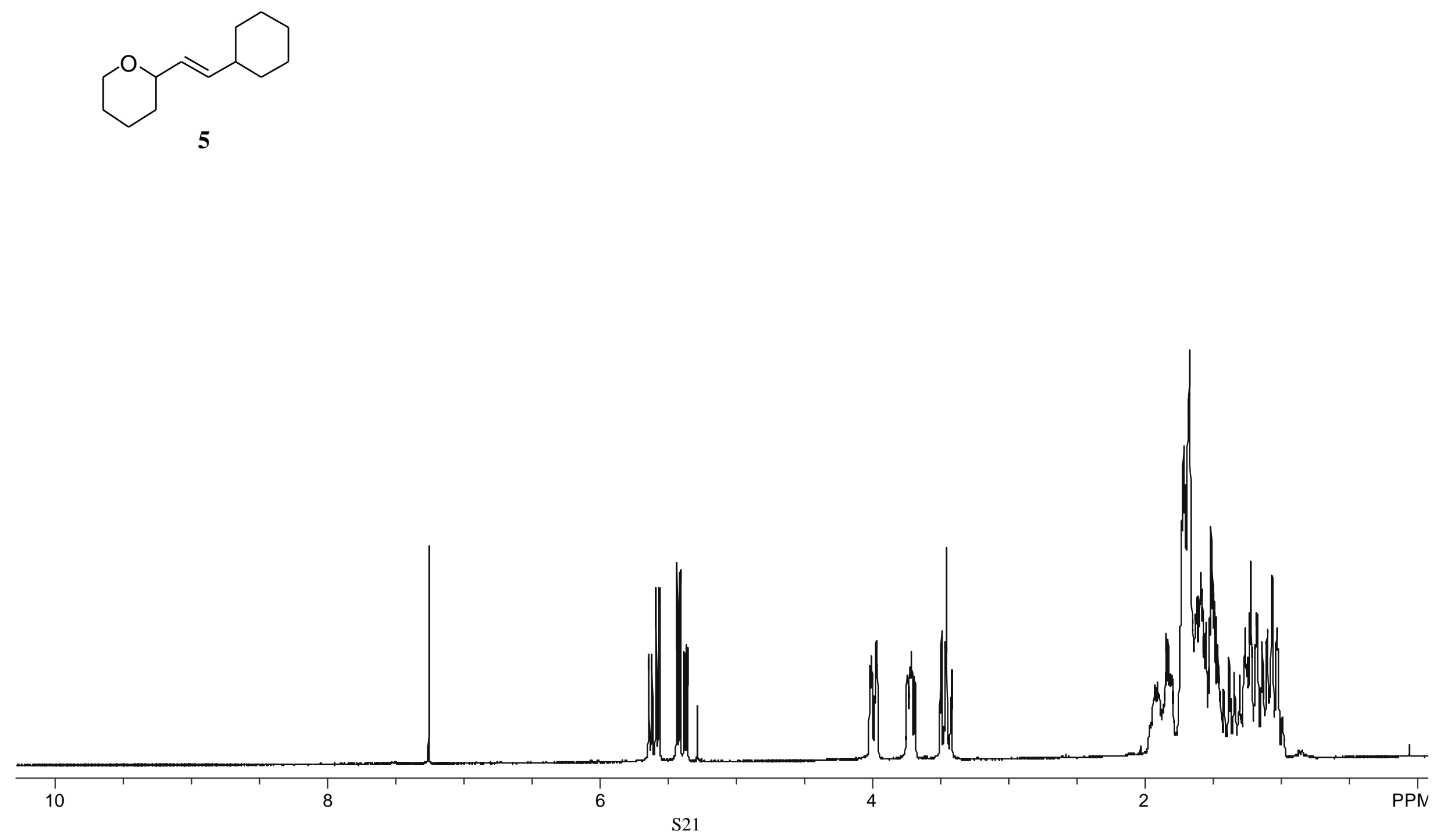


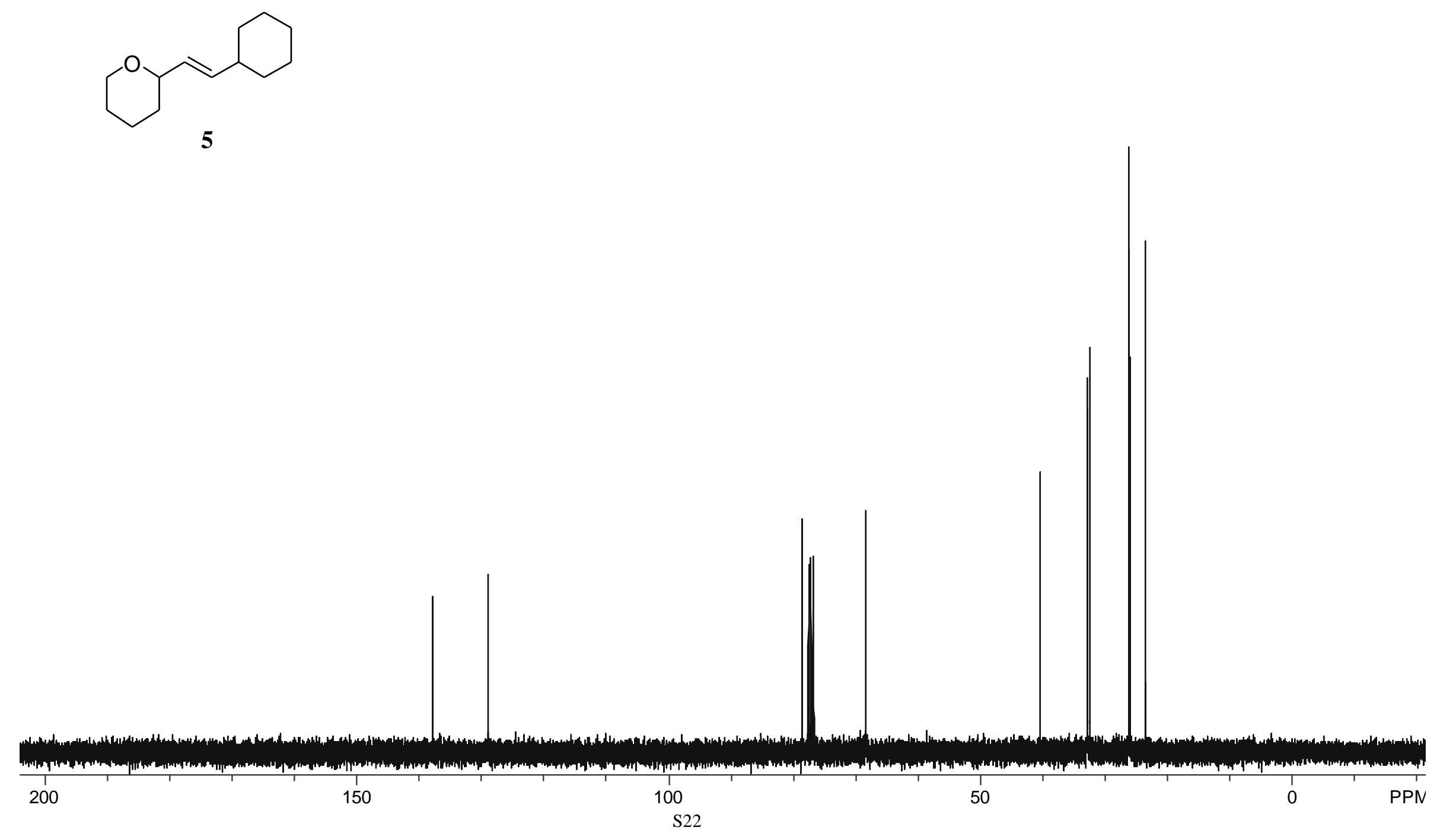



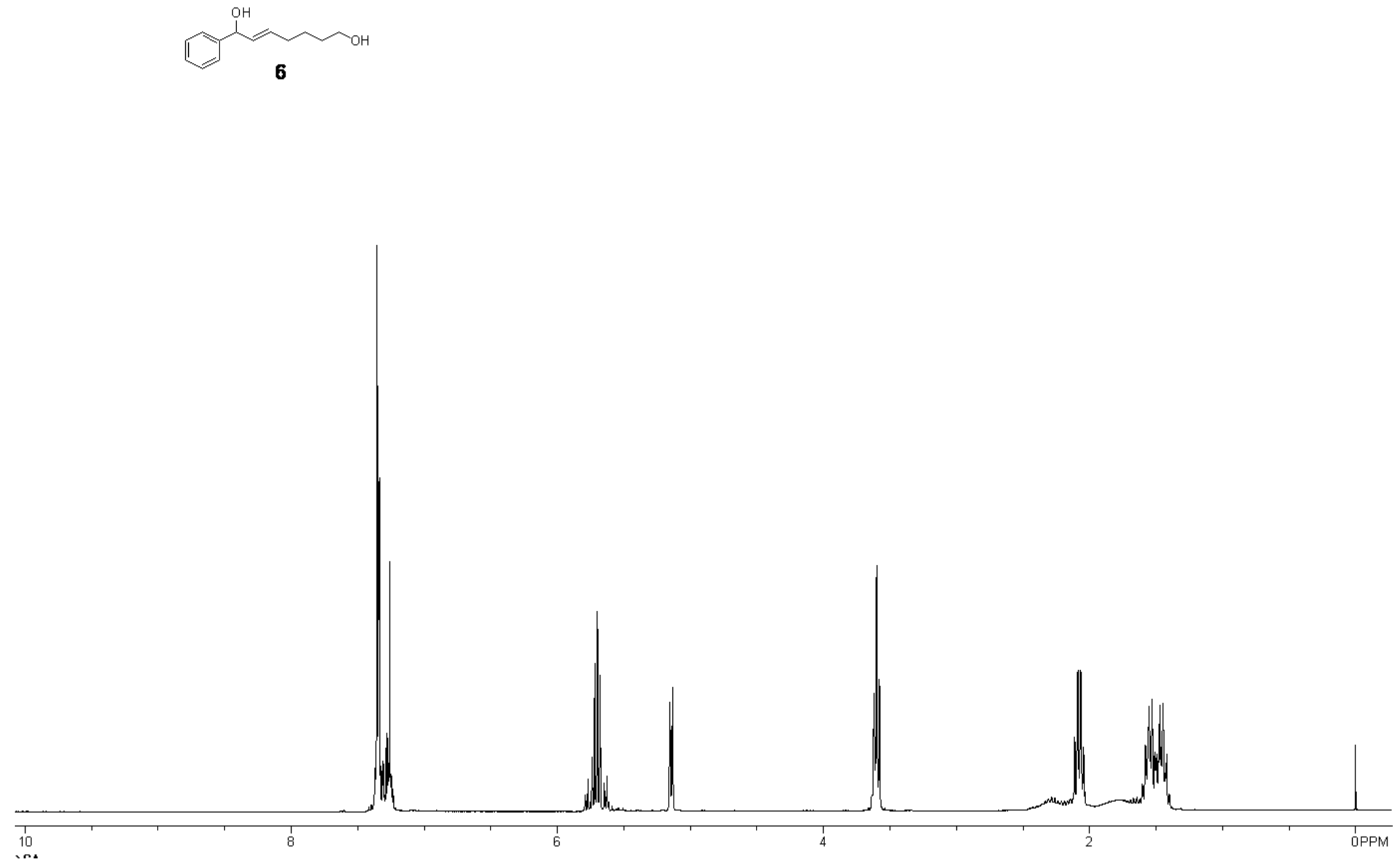

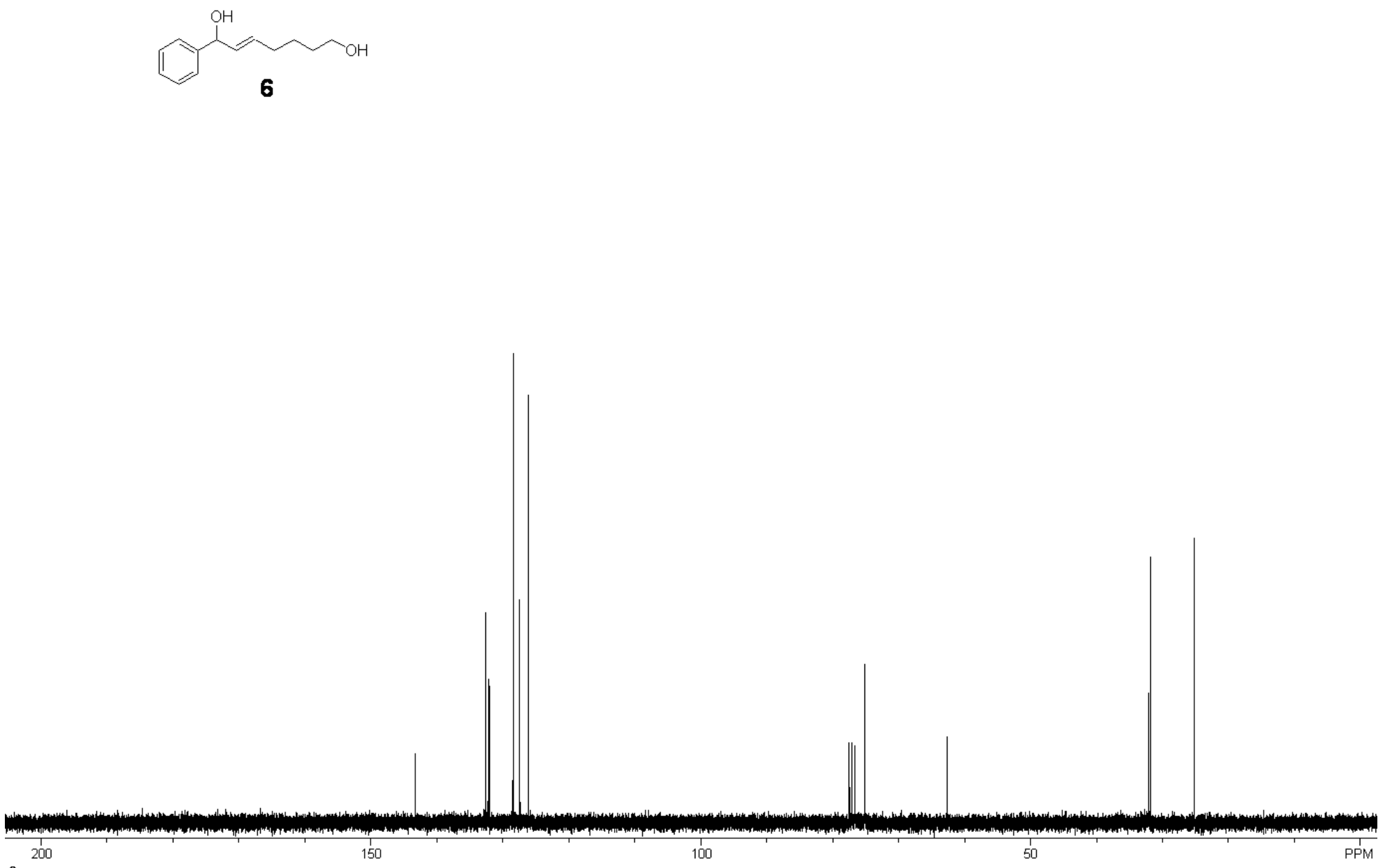

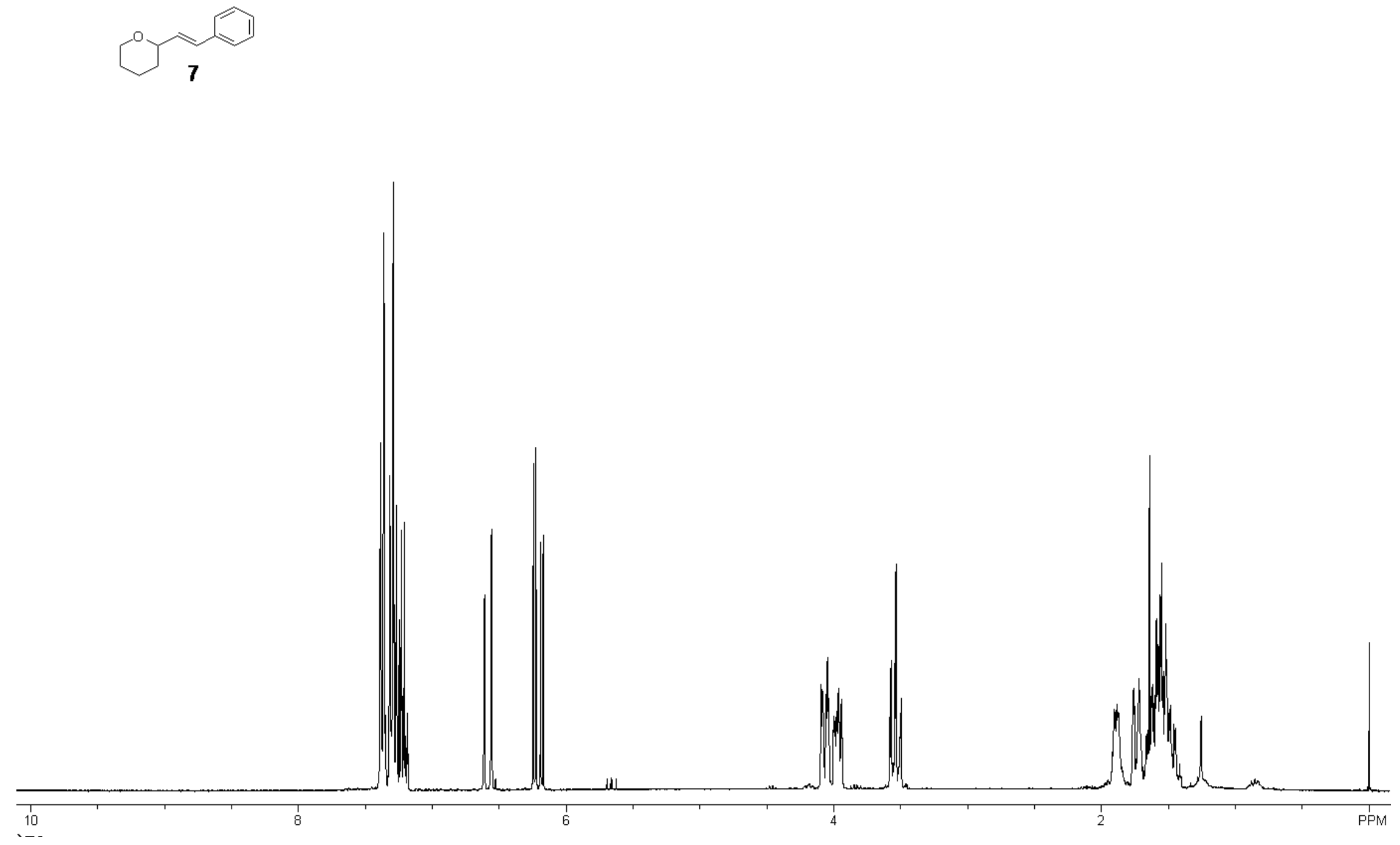

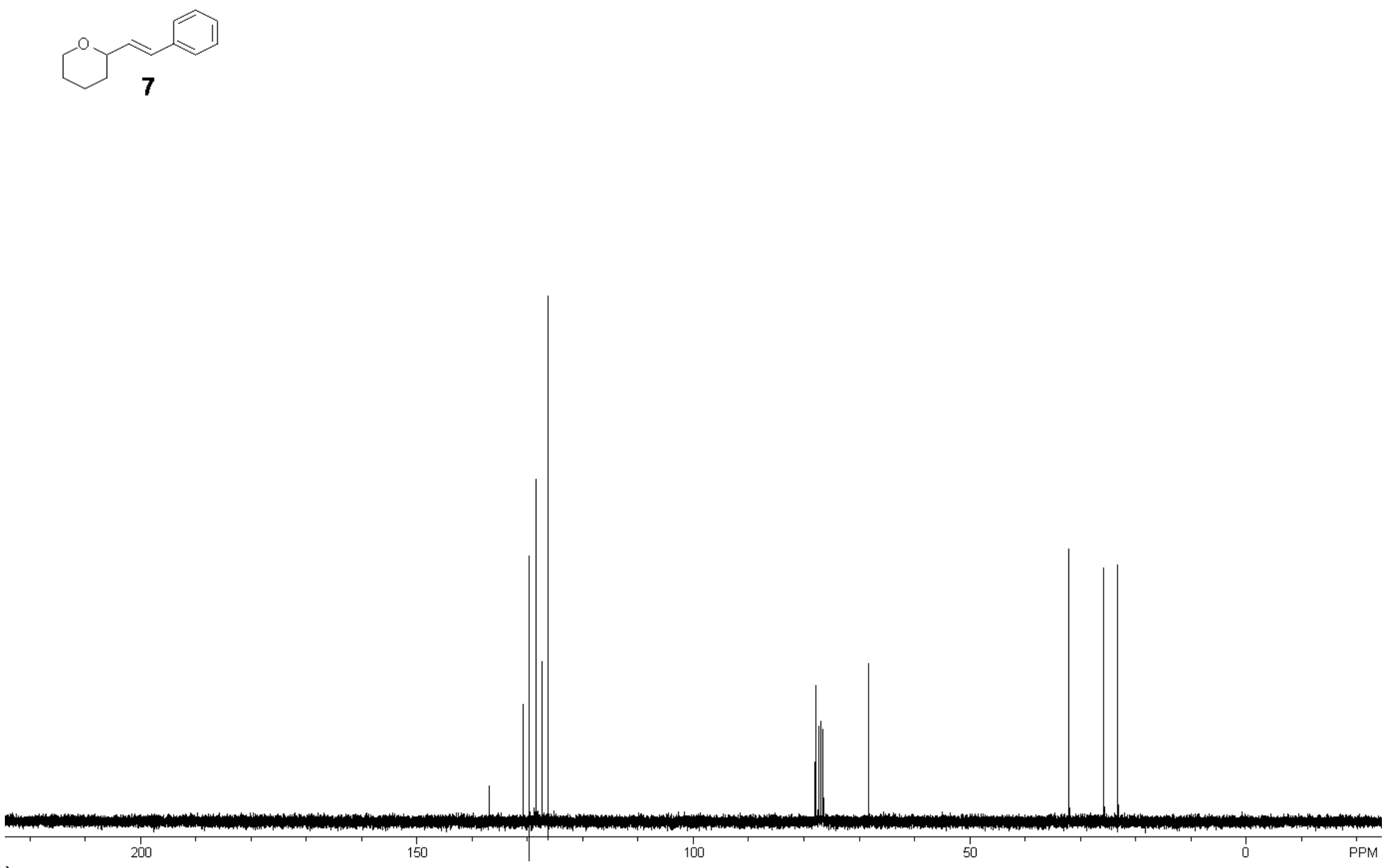

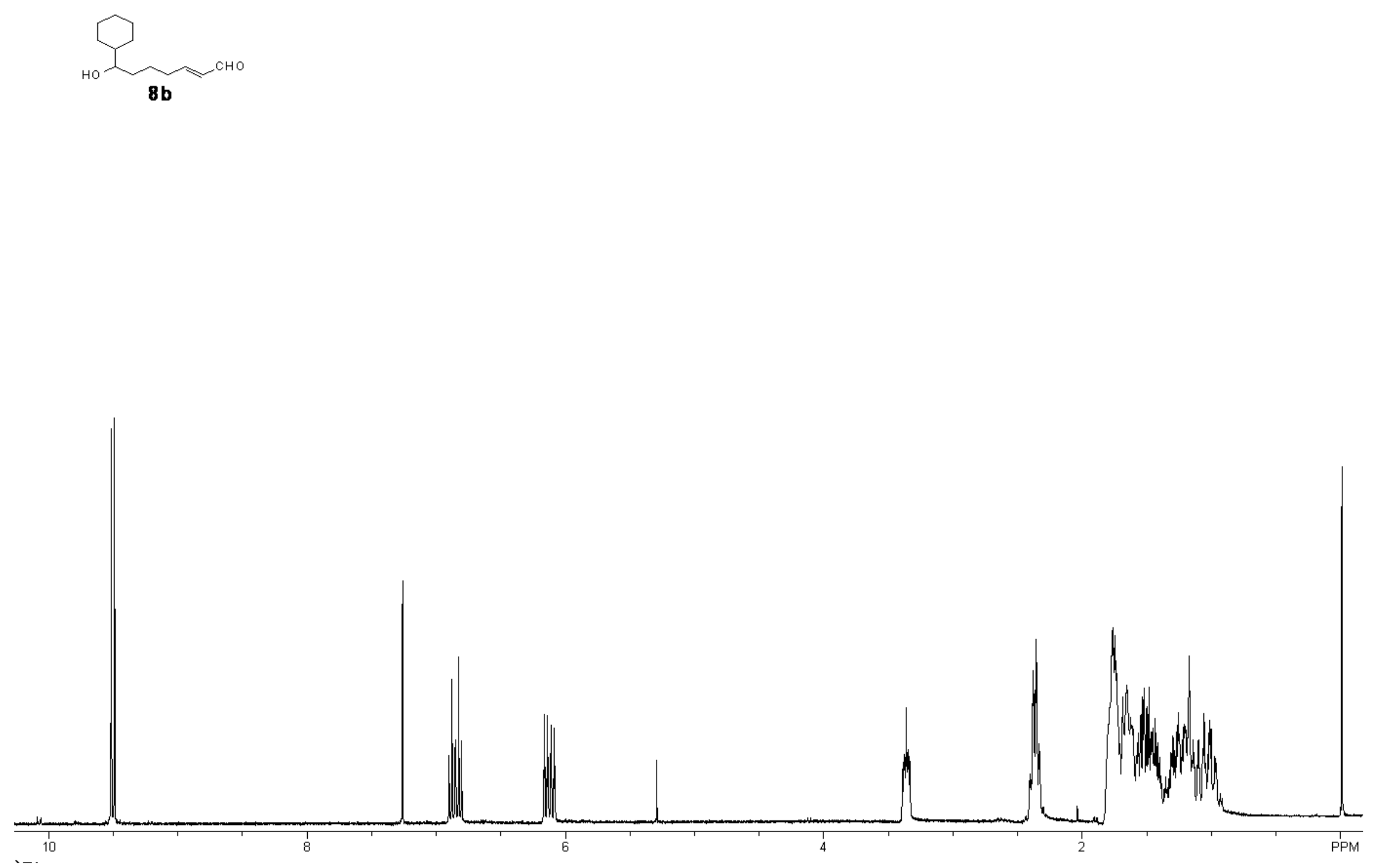

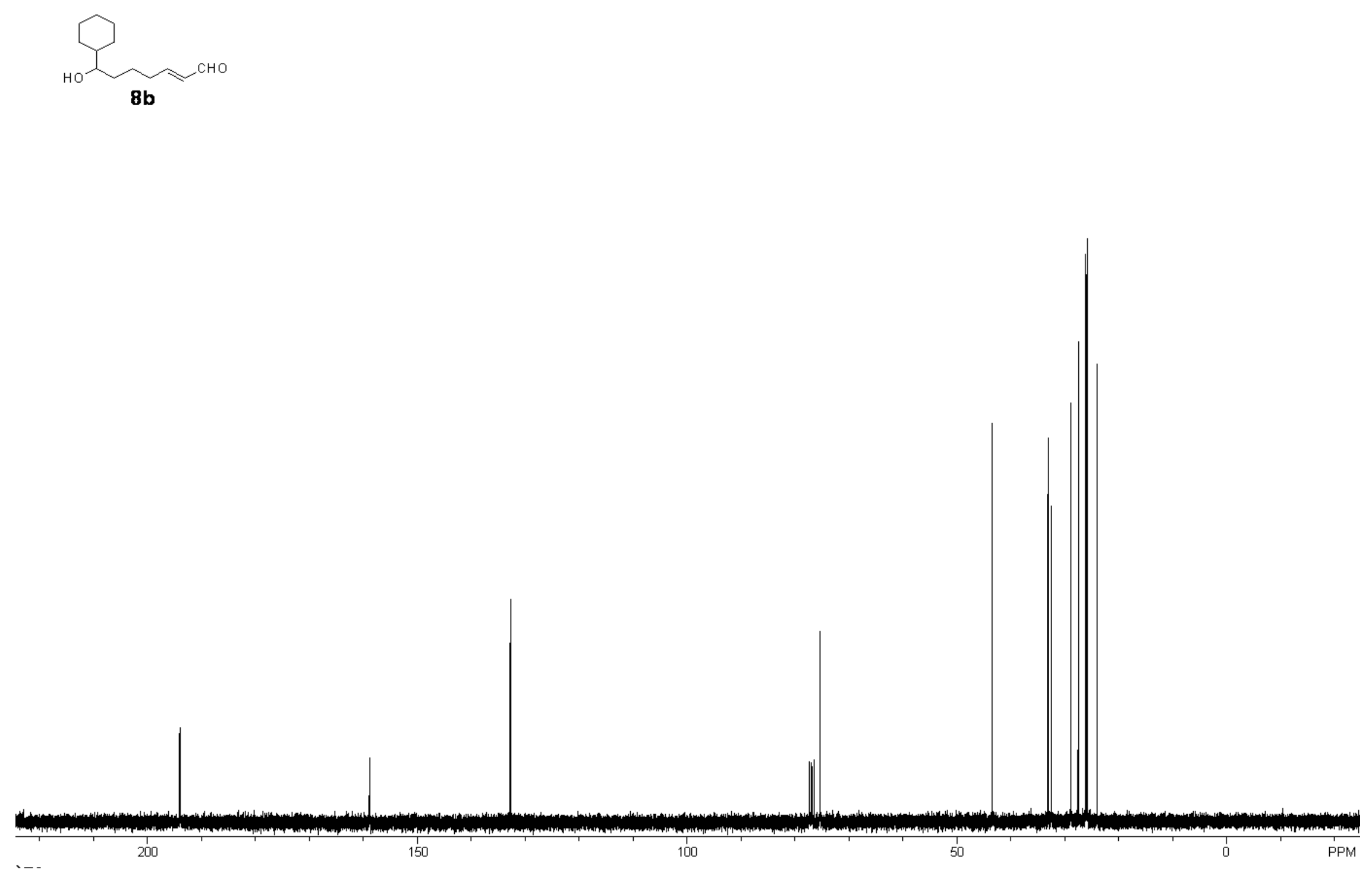
$\overbrace{8}{ }^{O H}$

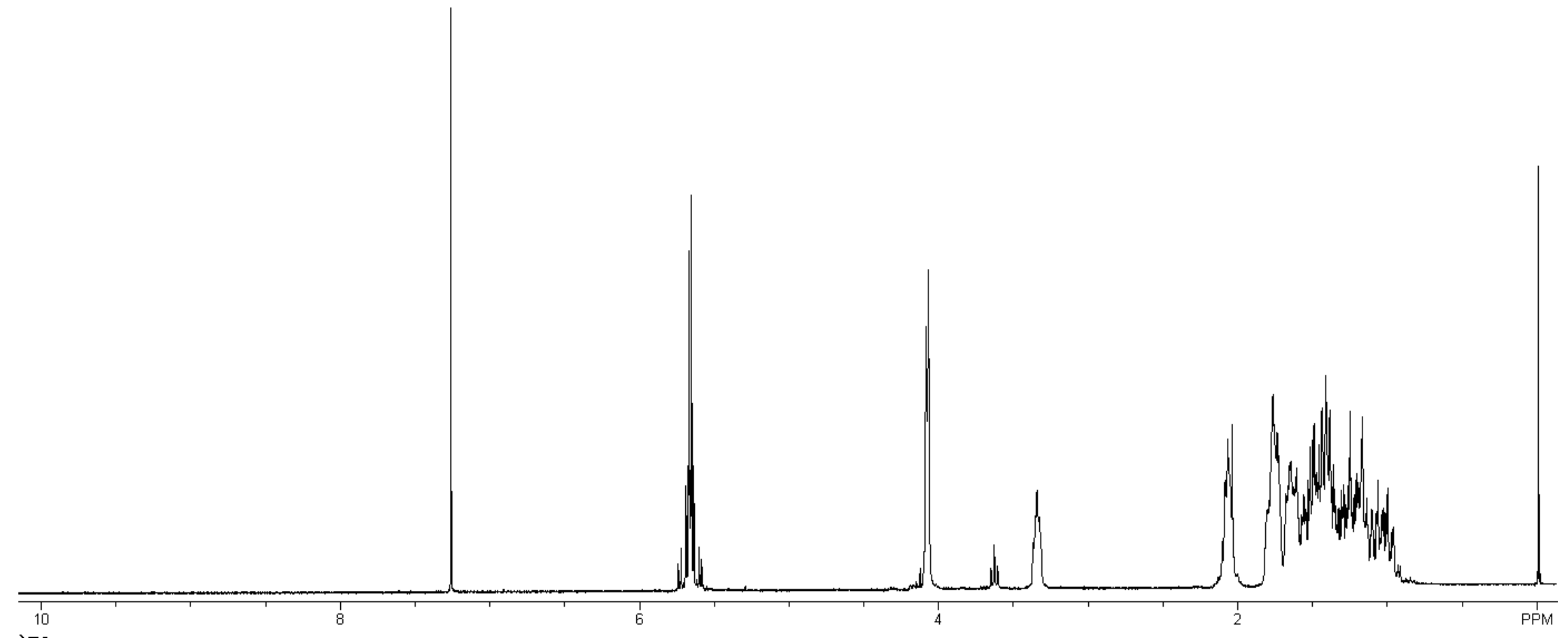



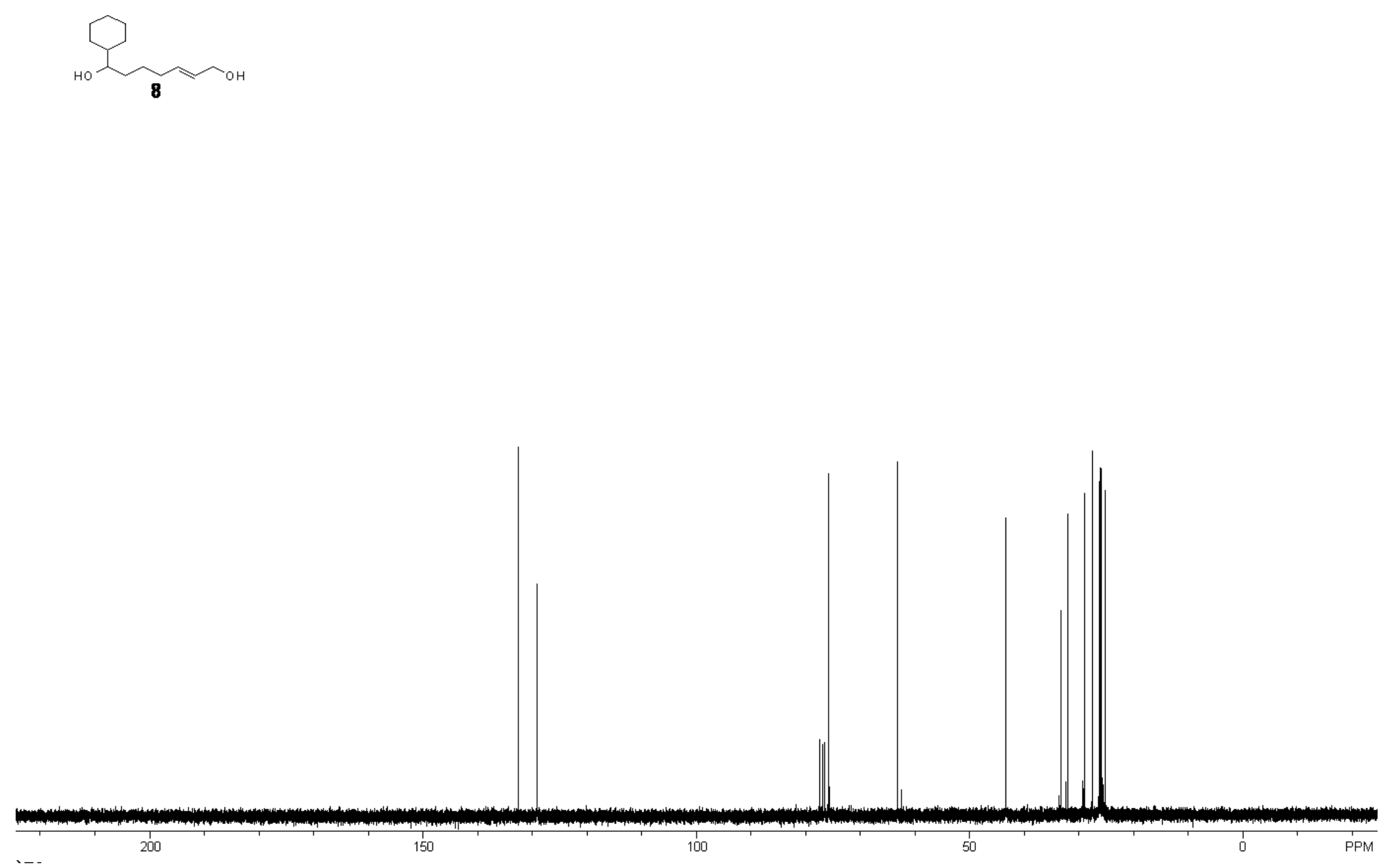
$\underbrace{\mathrm{C}_{6} \mathrm{H}_{11}}_{9}$

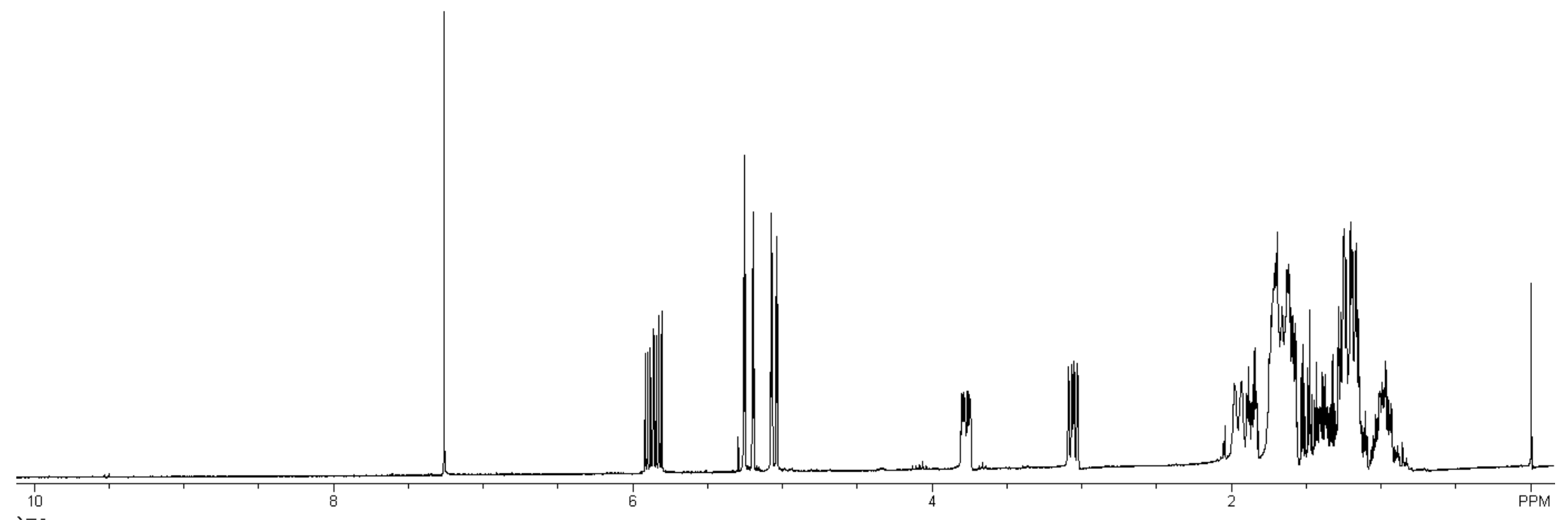


$\underbrace{\mathrm{C}_{6} \mathrm{H}_{11}}_{\mathbf{9}} \mathrm{C}^{\mathrm{O}}$

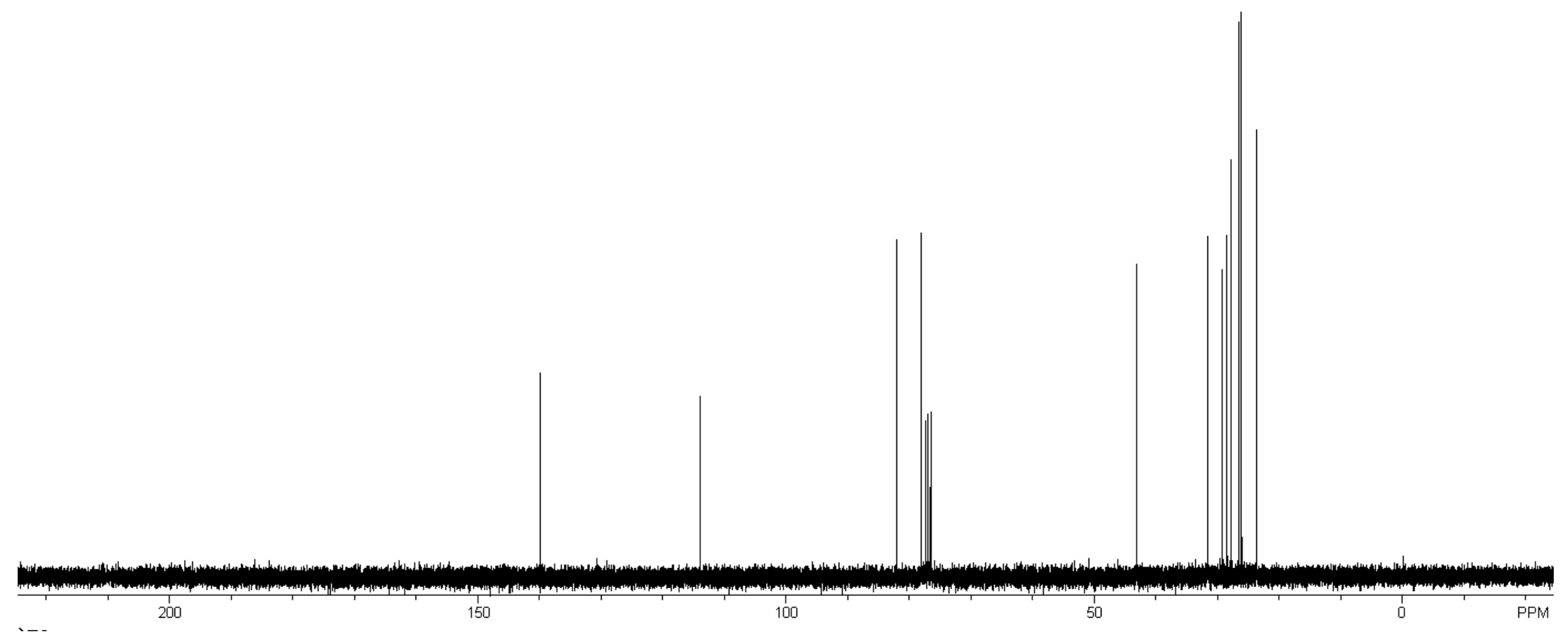



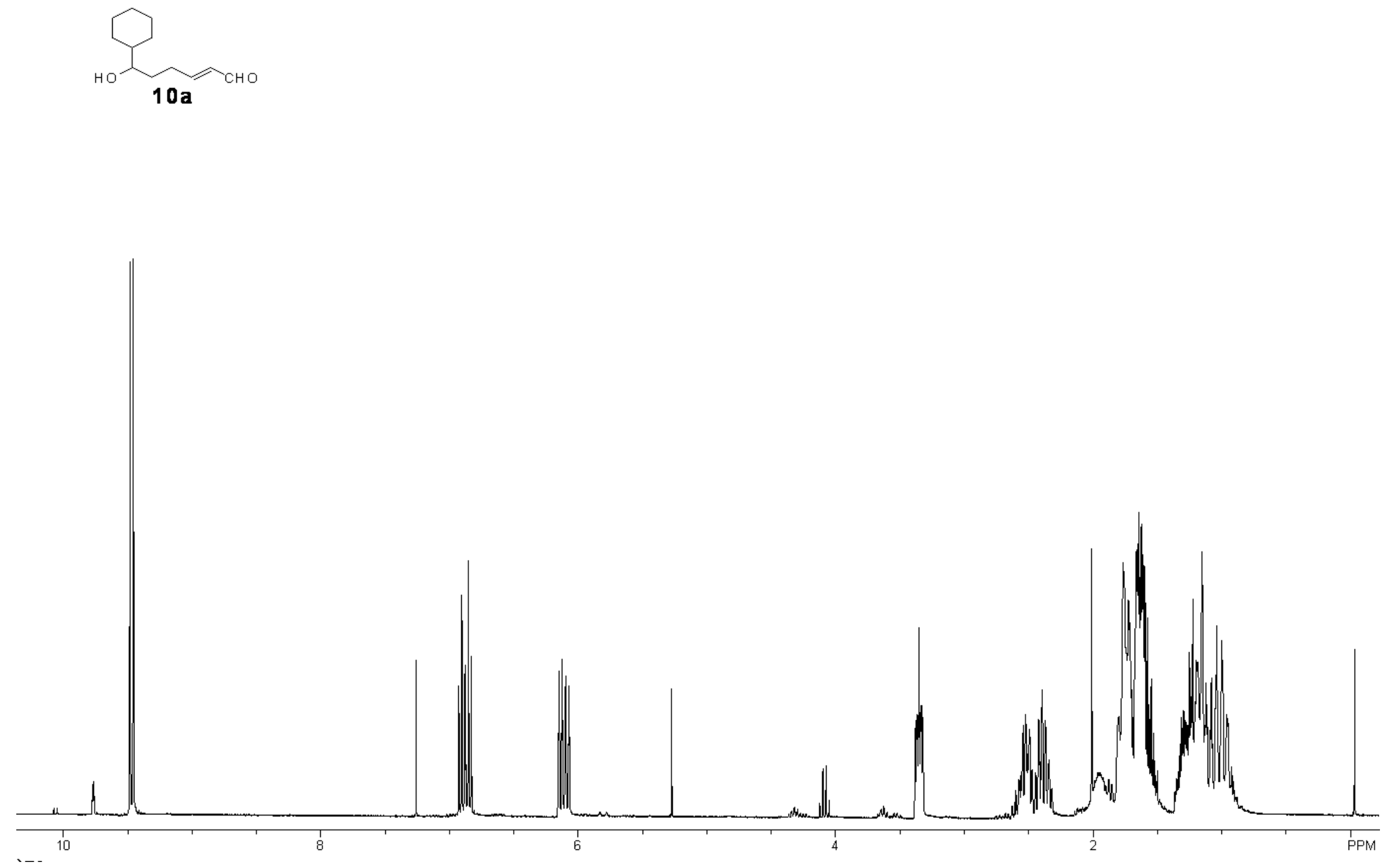

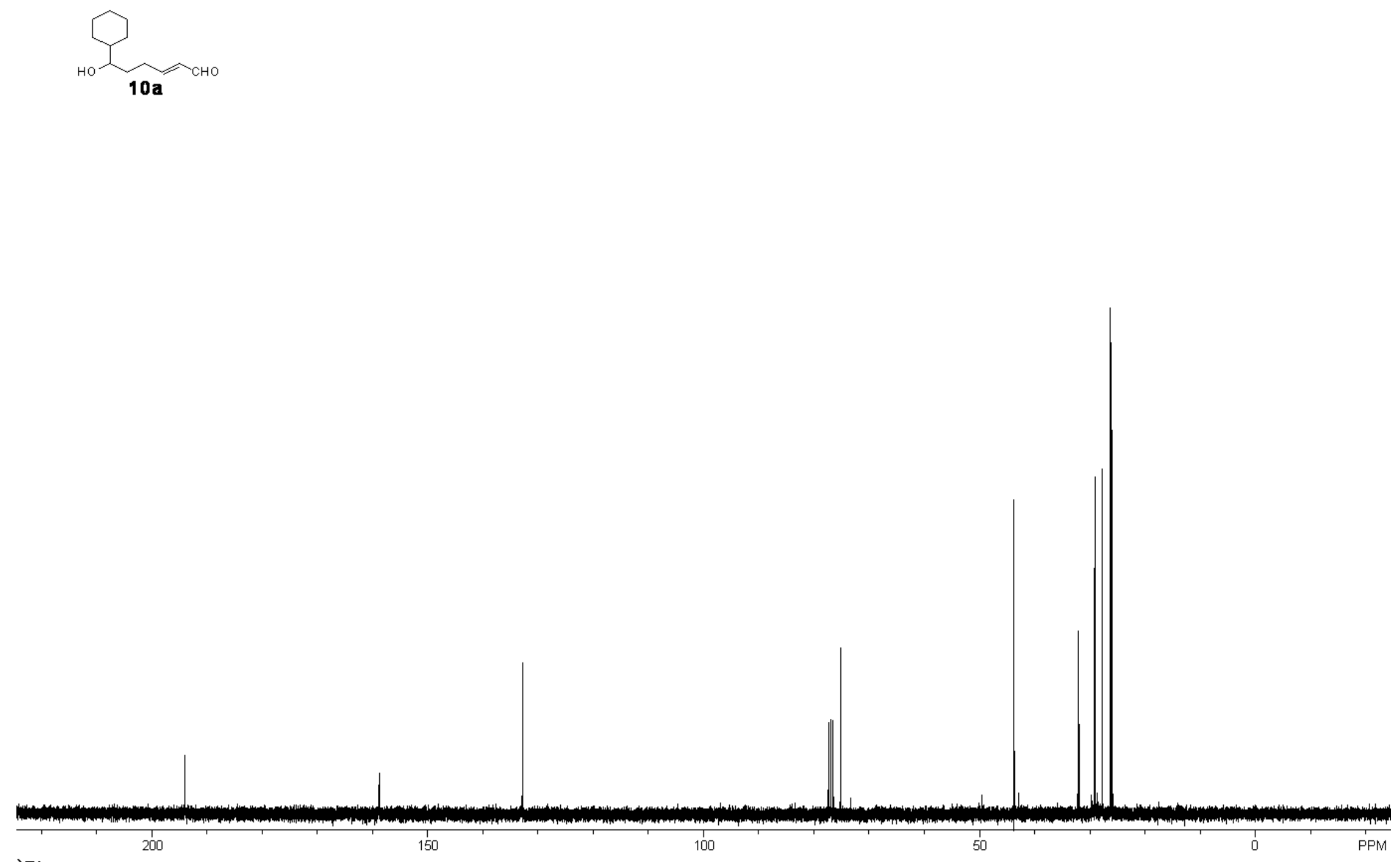

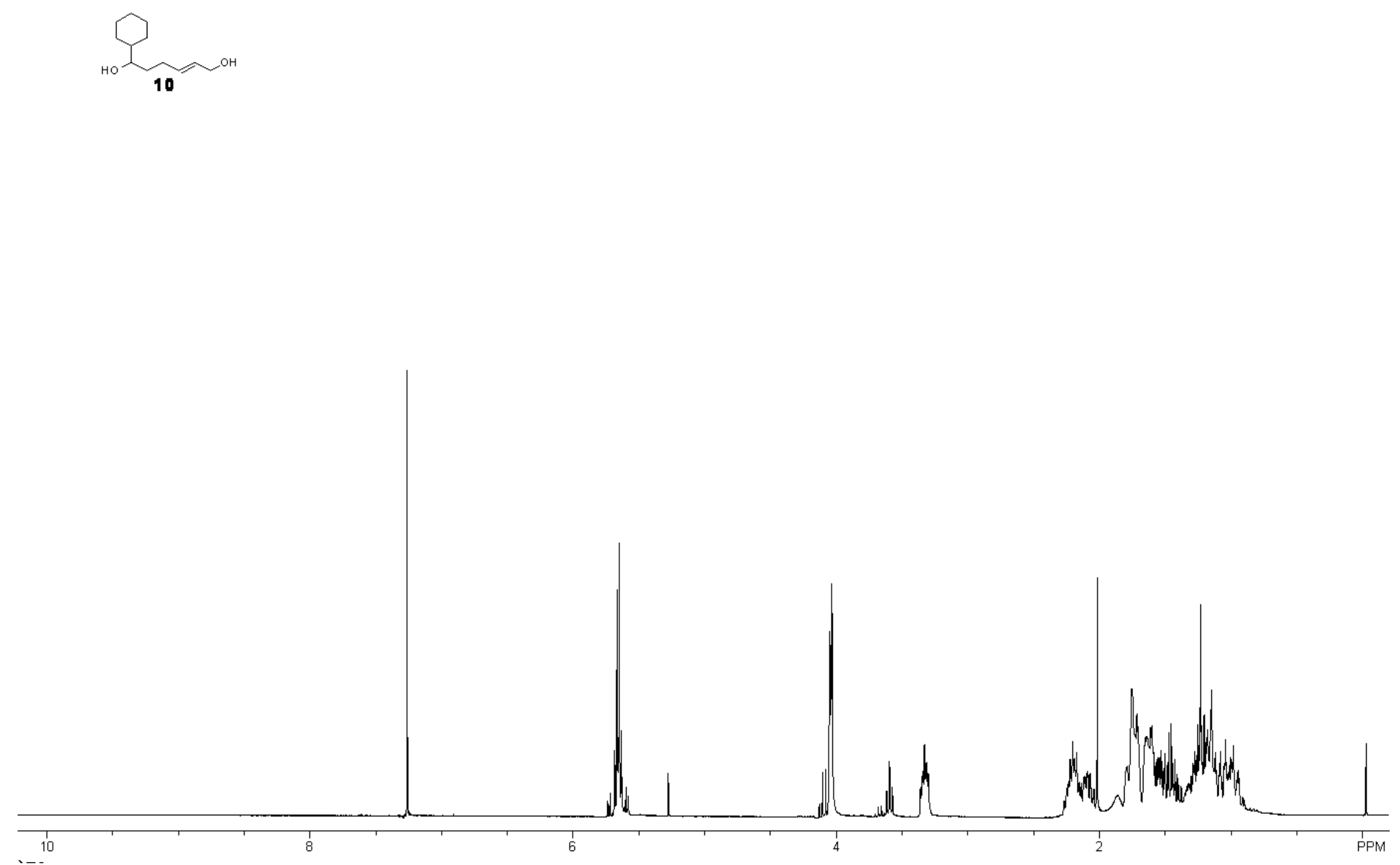

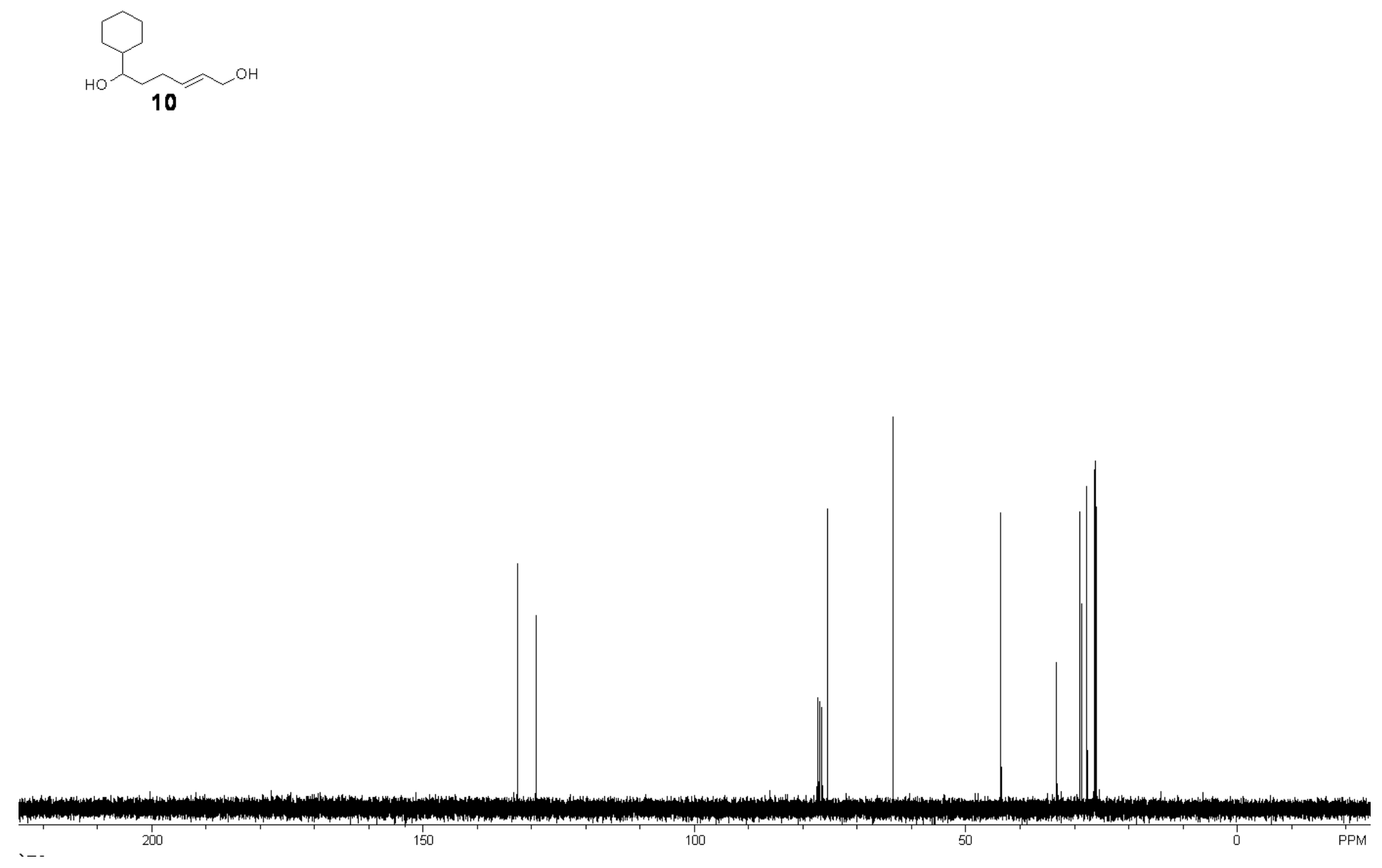

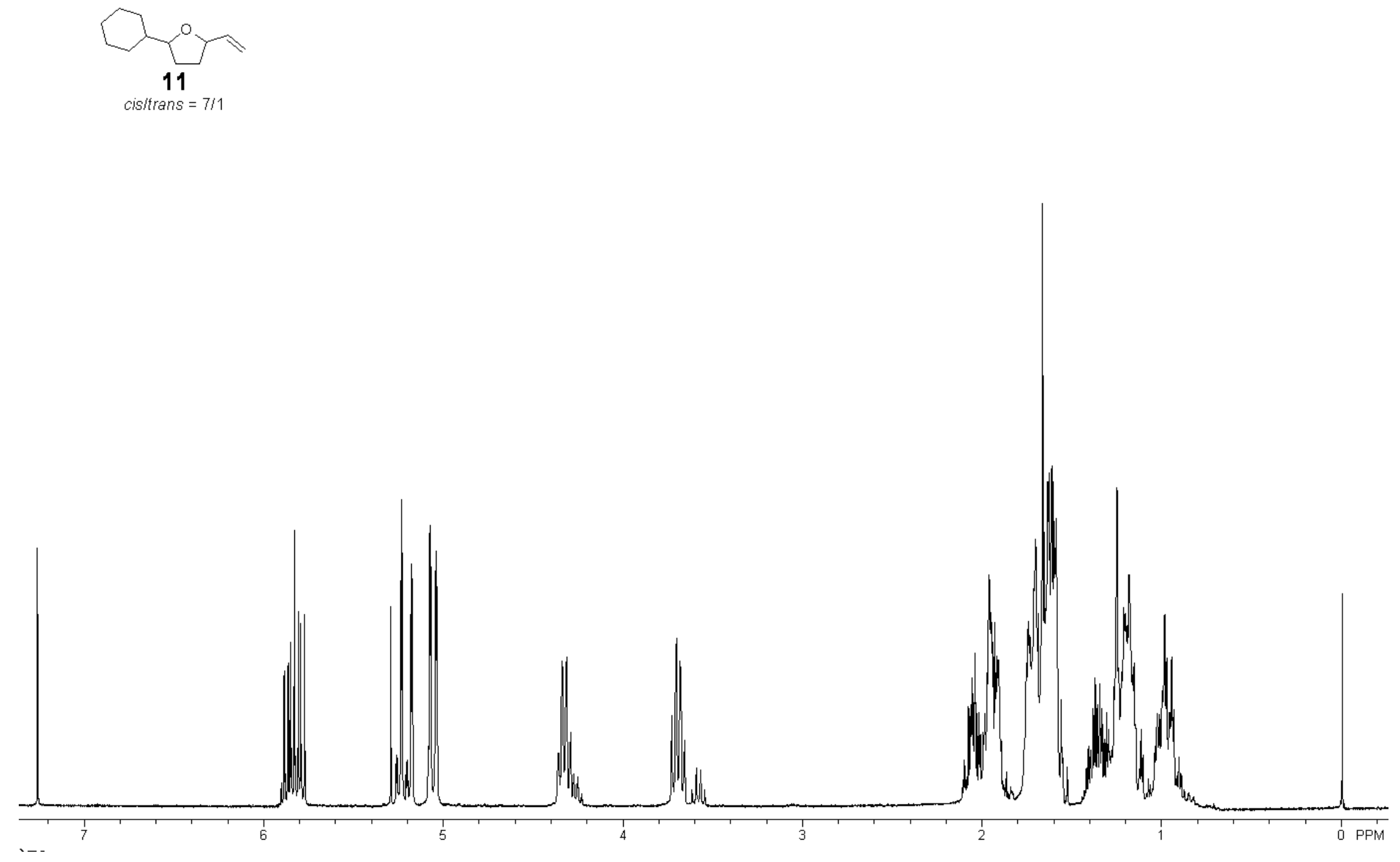


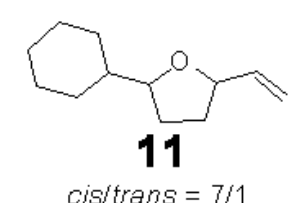

cis/trans $=7 / 1$

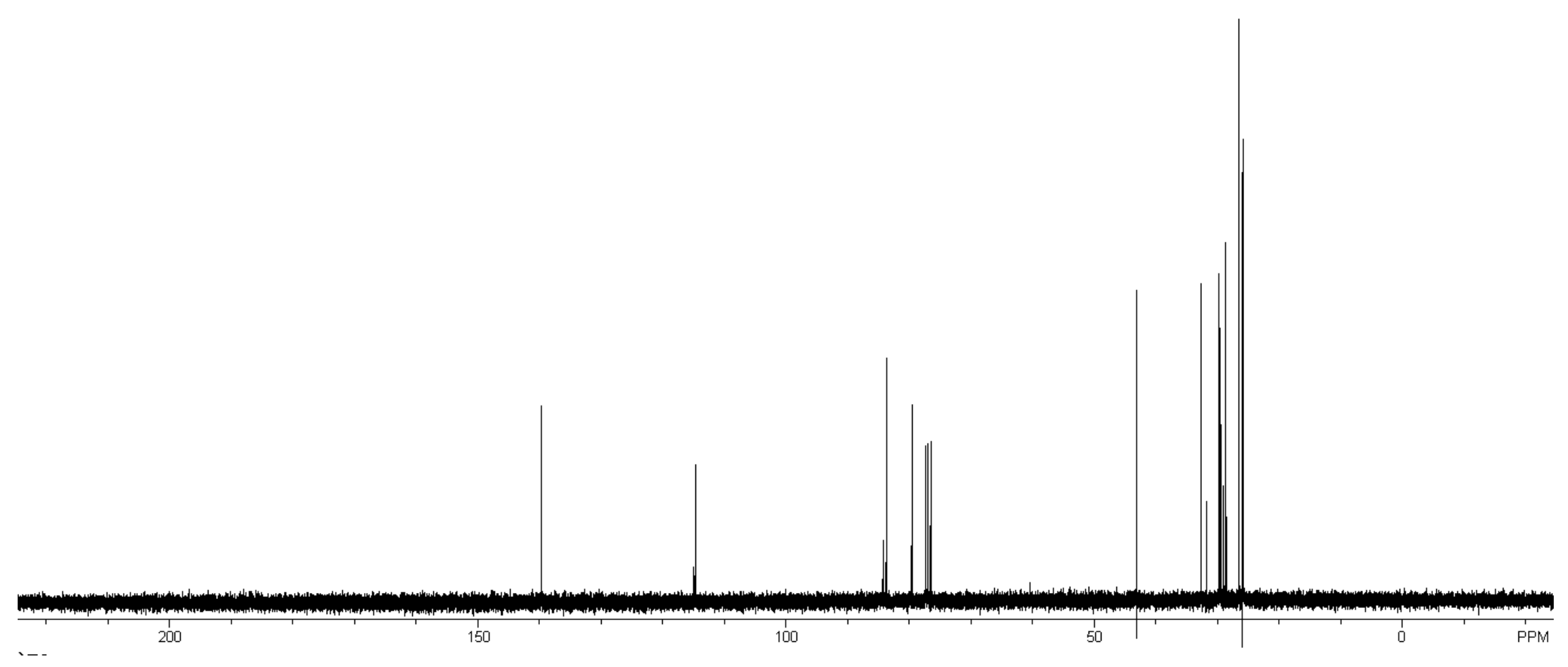




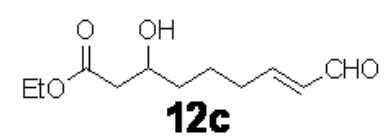

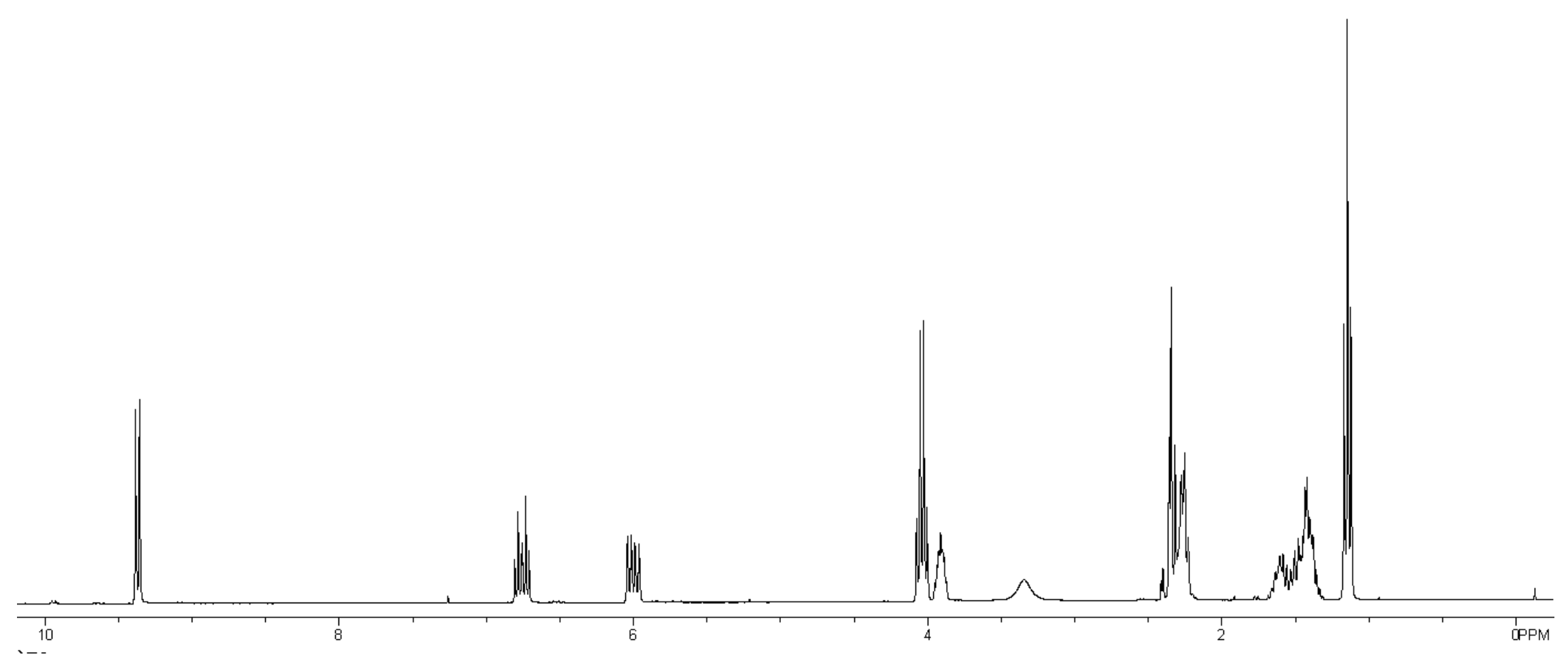




$$
\text { Eto } \underbrace{\mathrm{OH}}_{12 \mathrm{C}} \mathrm{CHO}
$$

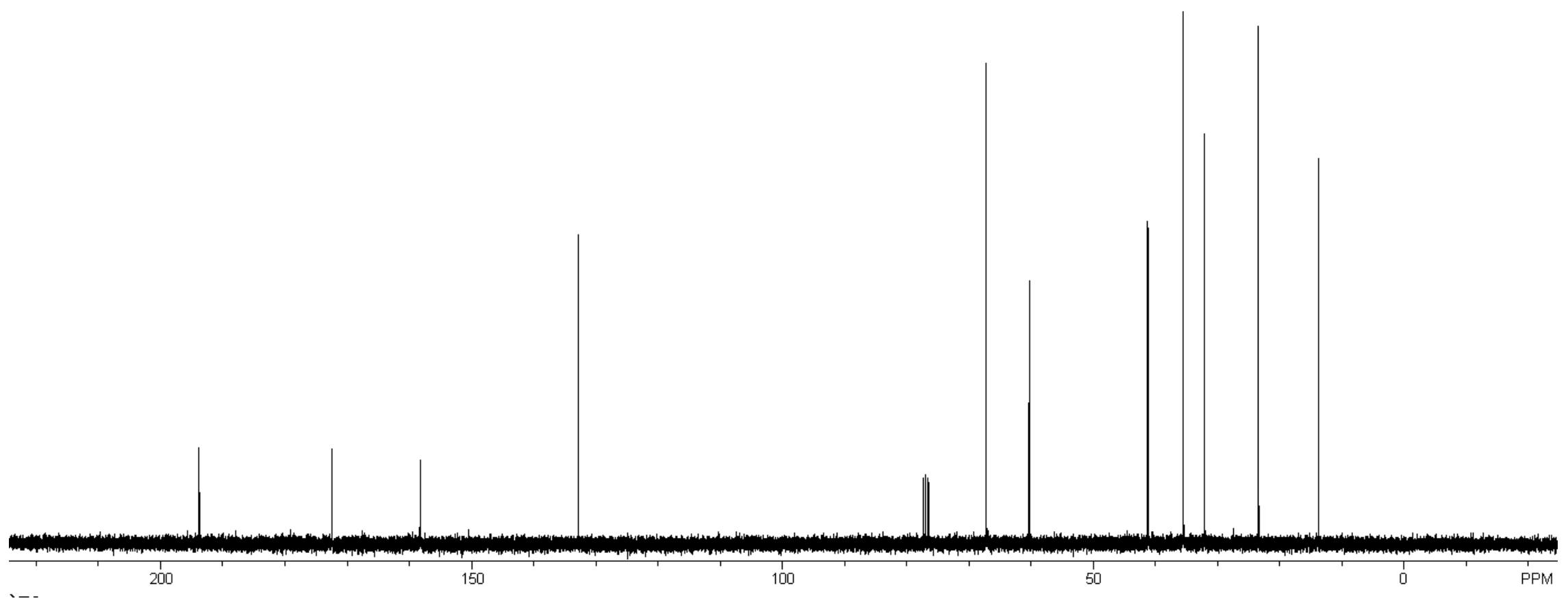


Eto $\overbrace{12}^{\stackrel{O H}{1}} \mathrm{COH}_{\mathrm{OH}}$

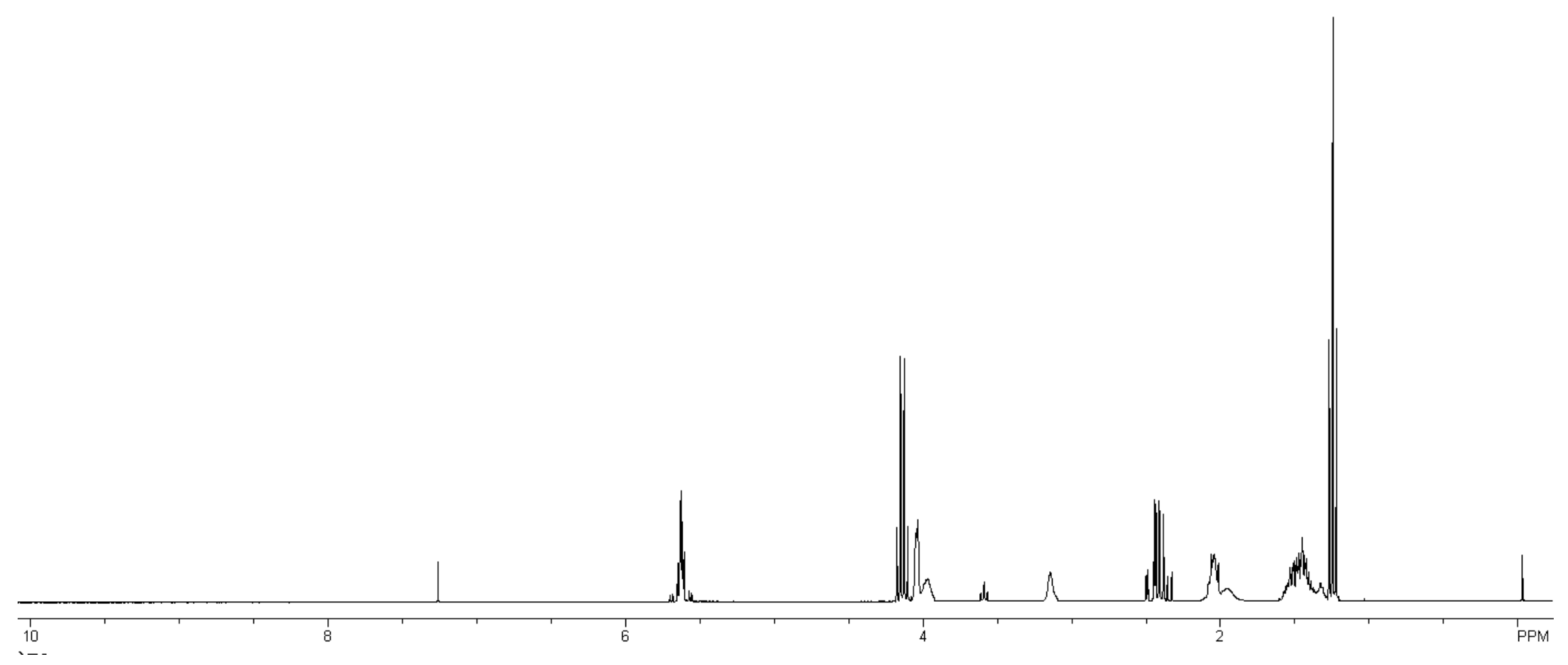




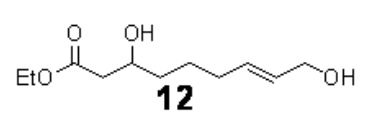

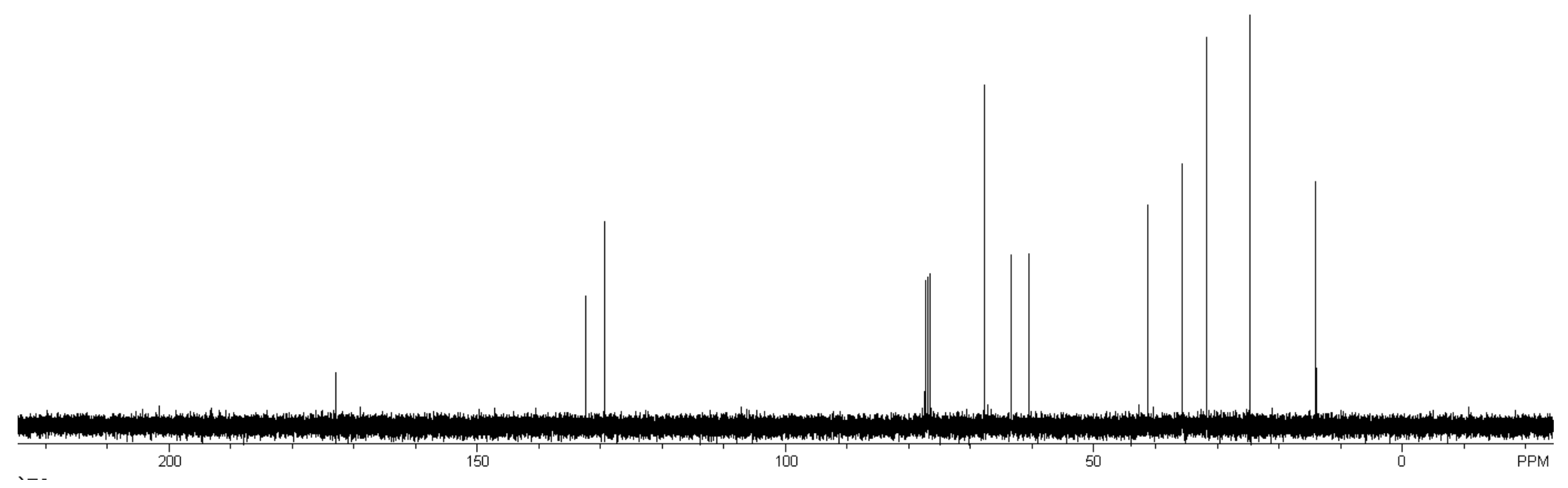


Etooc $\bigcup_{13 a}^{0}$

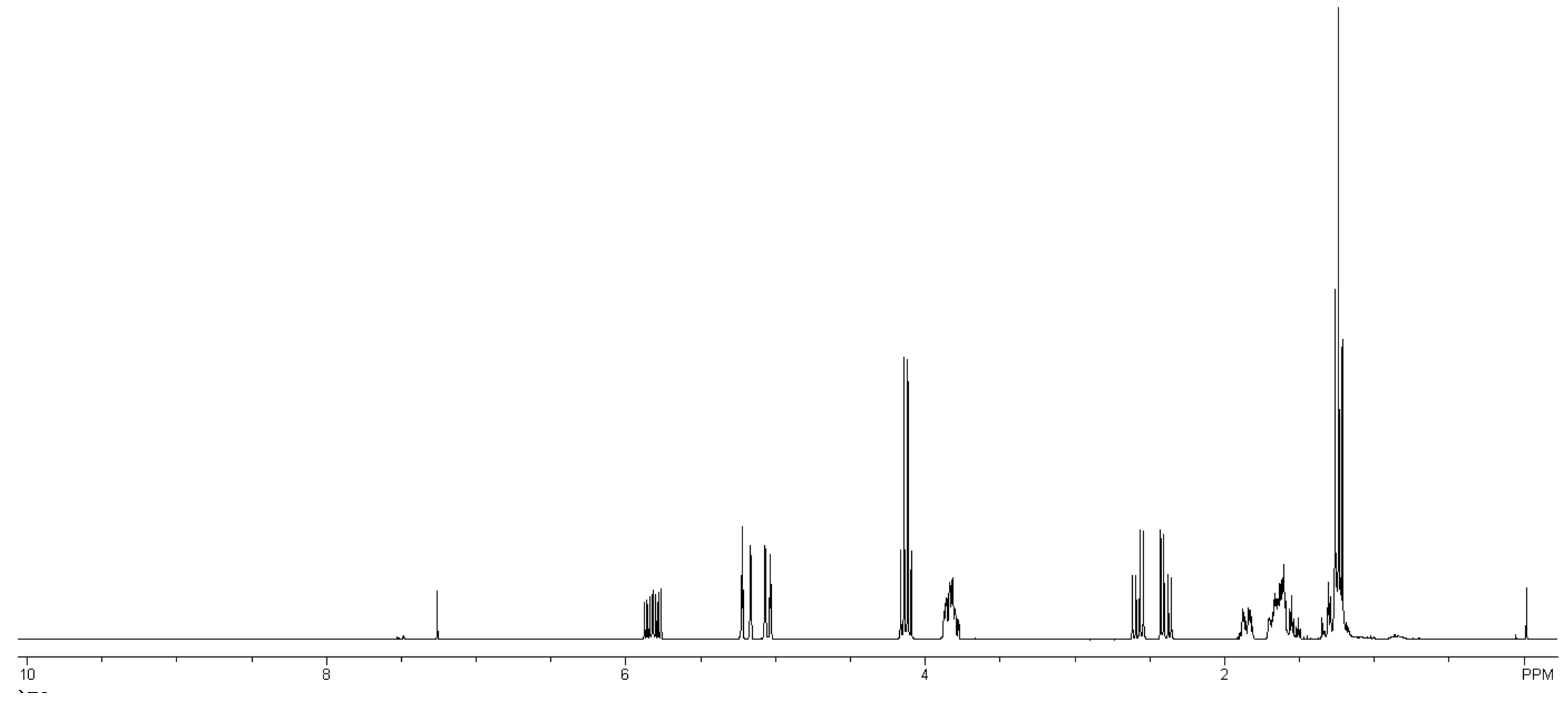


Etooc 0

$13 a$

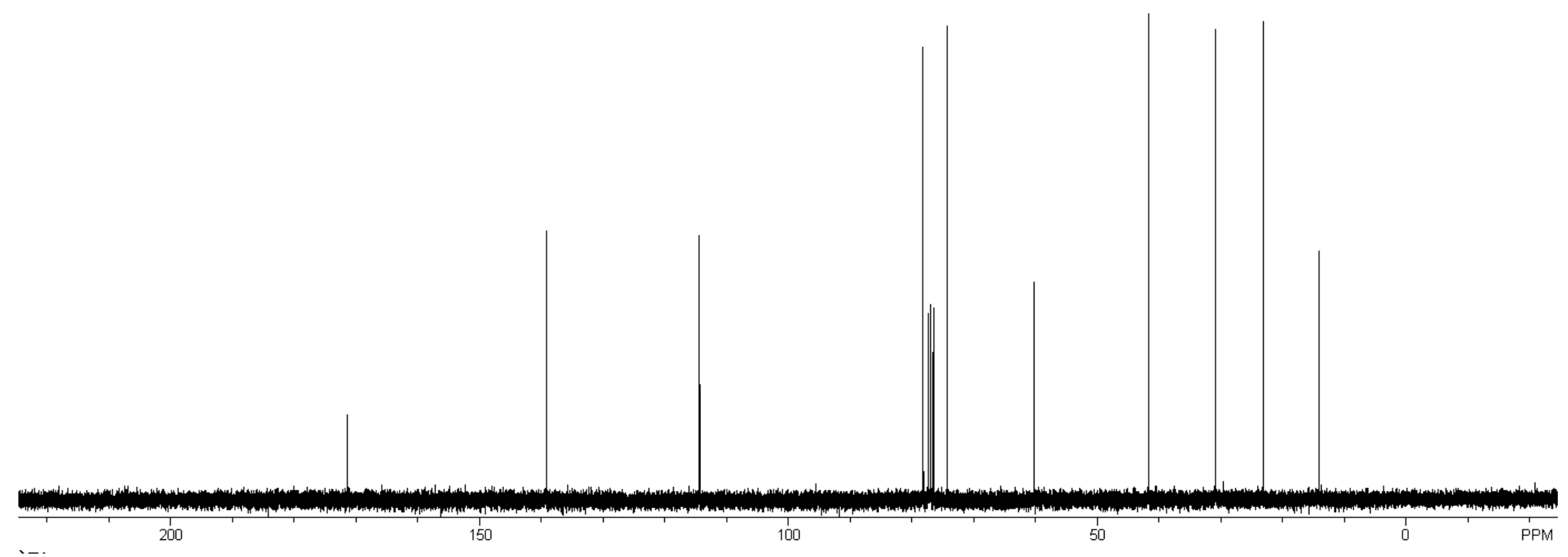




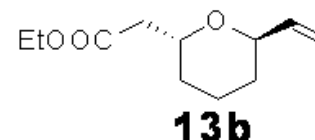

$13 b$

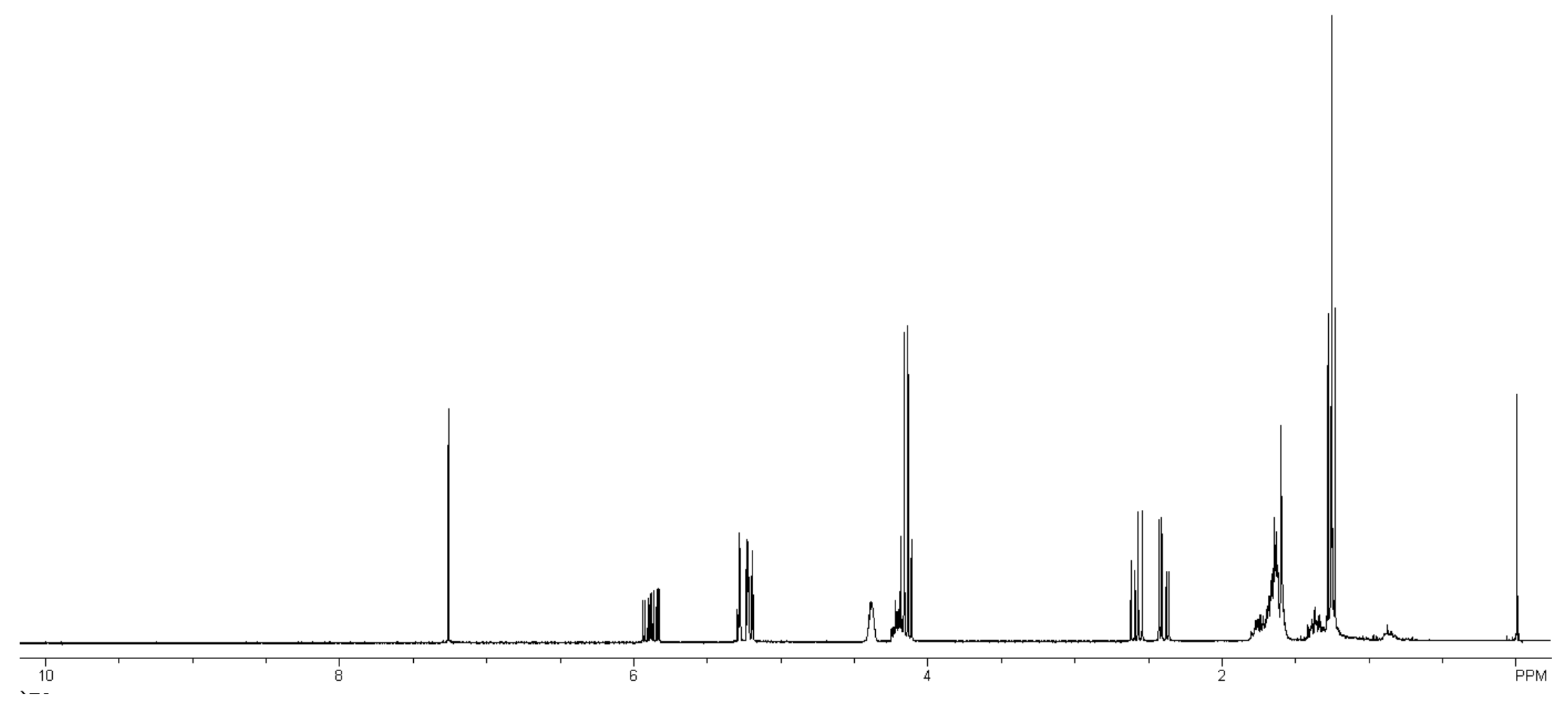


Etooc"

$13 b$

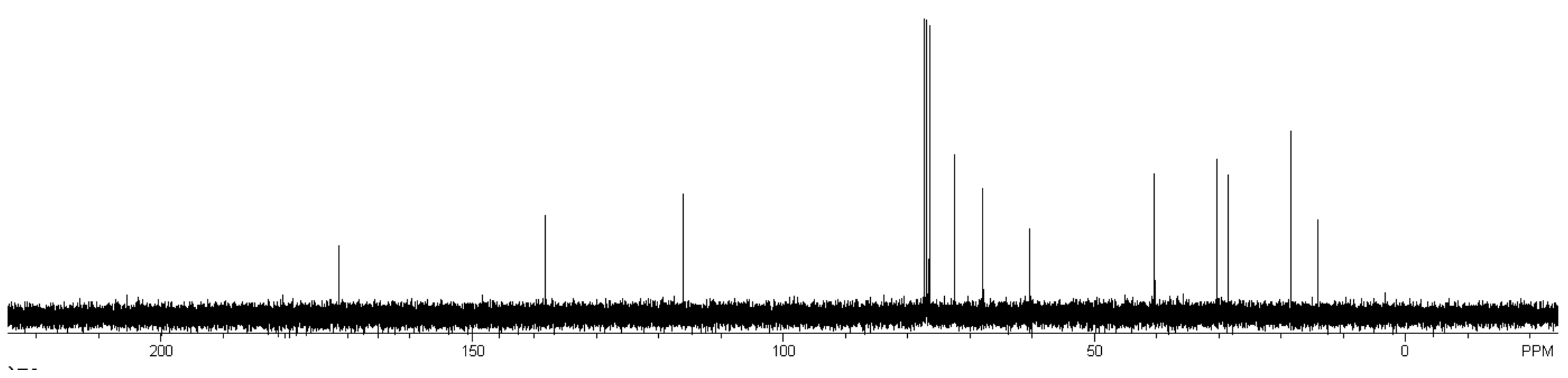


$\overbrace{14 a}{ }^{\circ}$

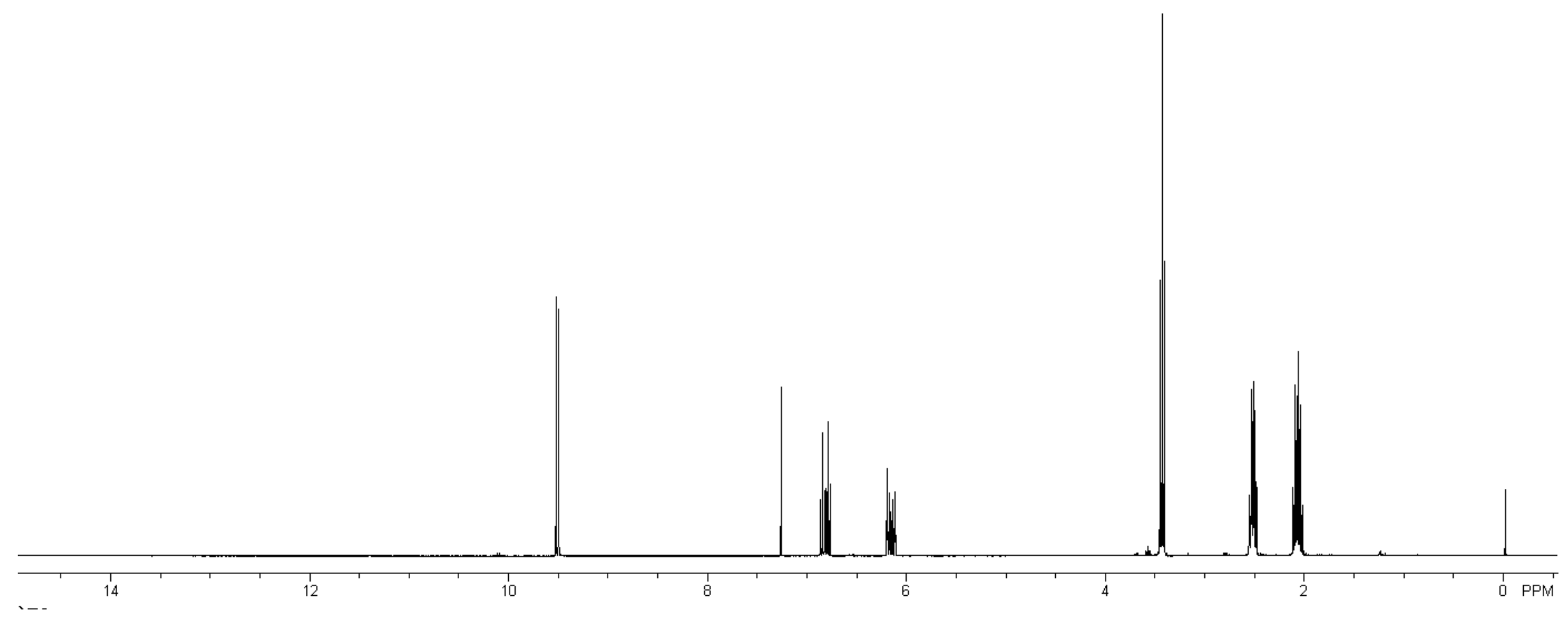


$\mathrm{Br} \sim \curvearrowright_{0}$

$14 a$

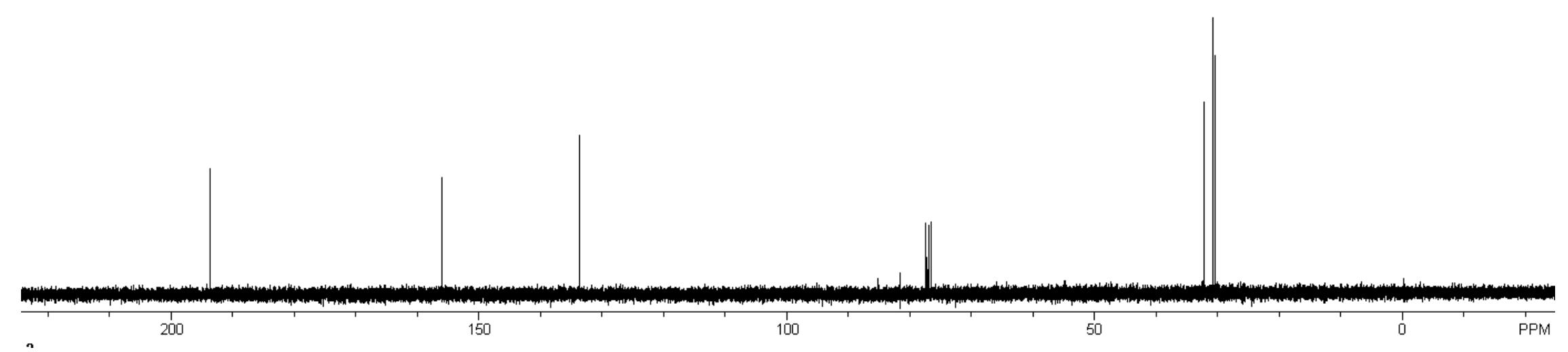


$\overbrace{14 \mathrm{C}}^{\mathrm{Br}}$ OTBS

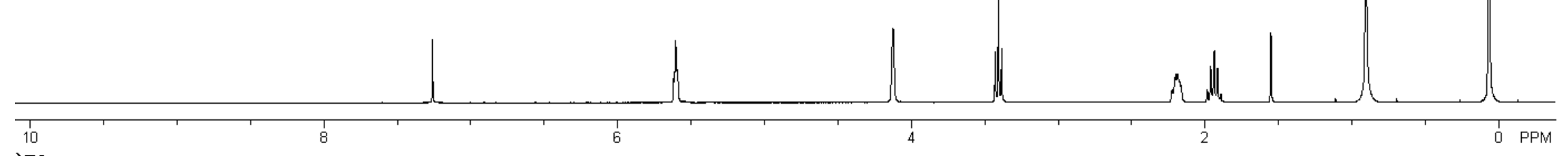


$\mathrm{Br} \sim$ OTBS

14c

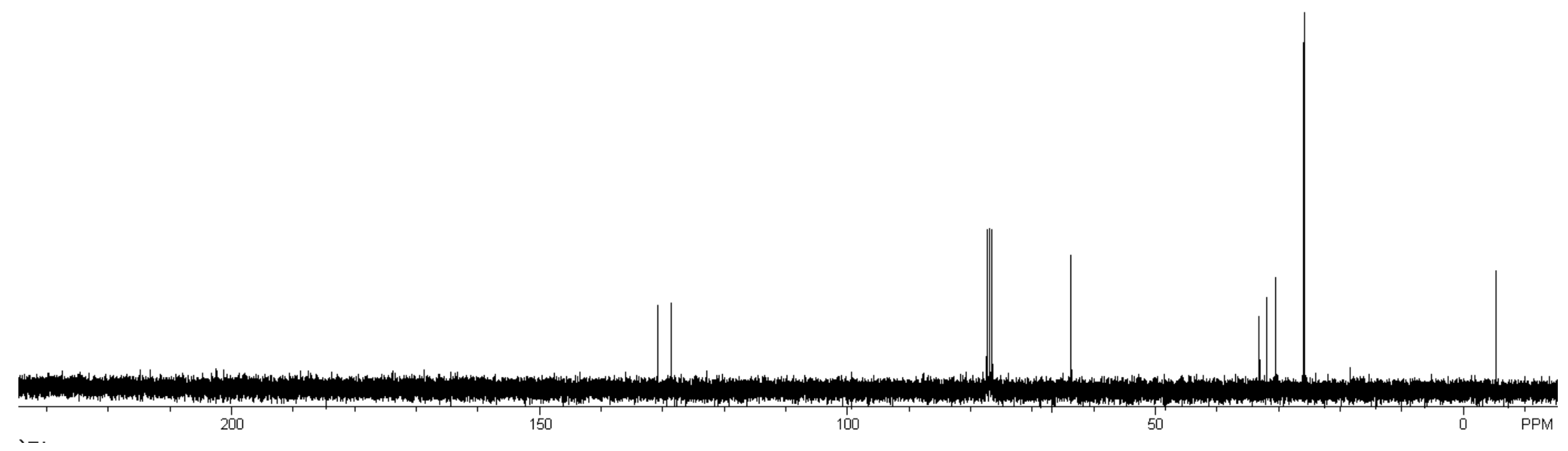


$\mathrm{OH}$

BnO $\curvearrowright$ OTBS

14d

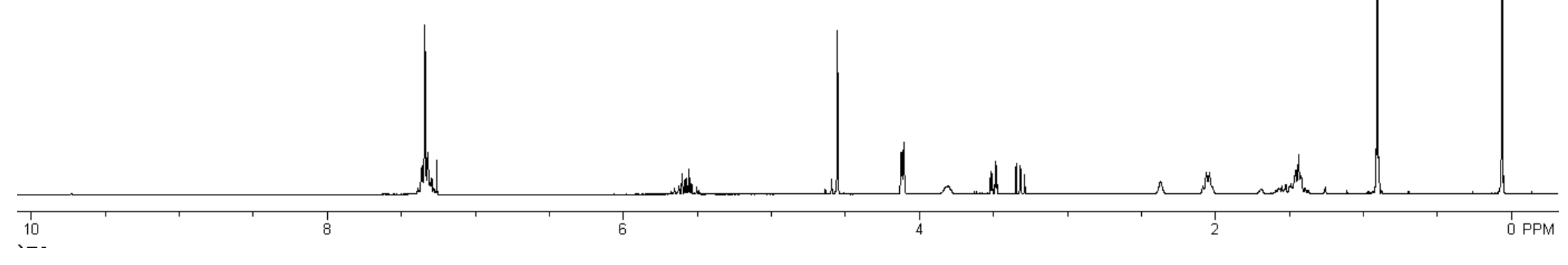




$$
\text { 14d }
$$

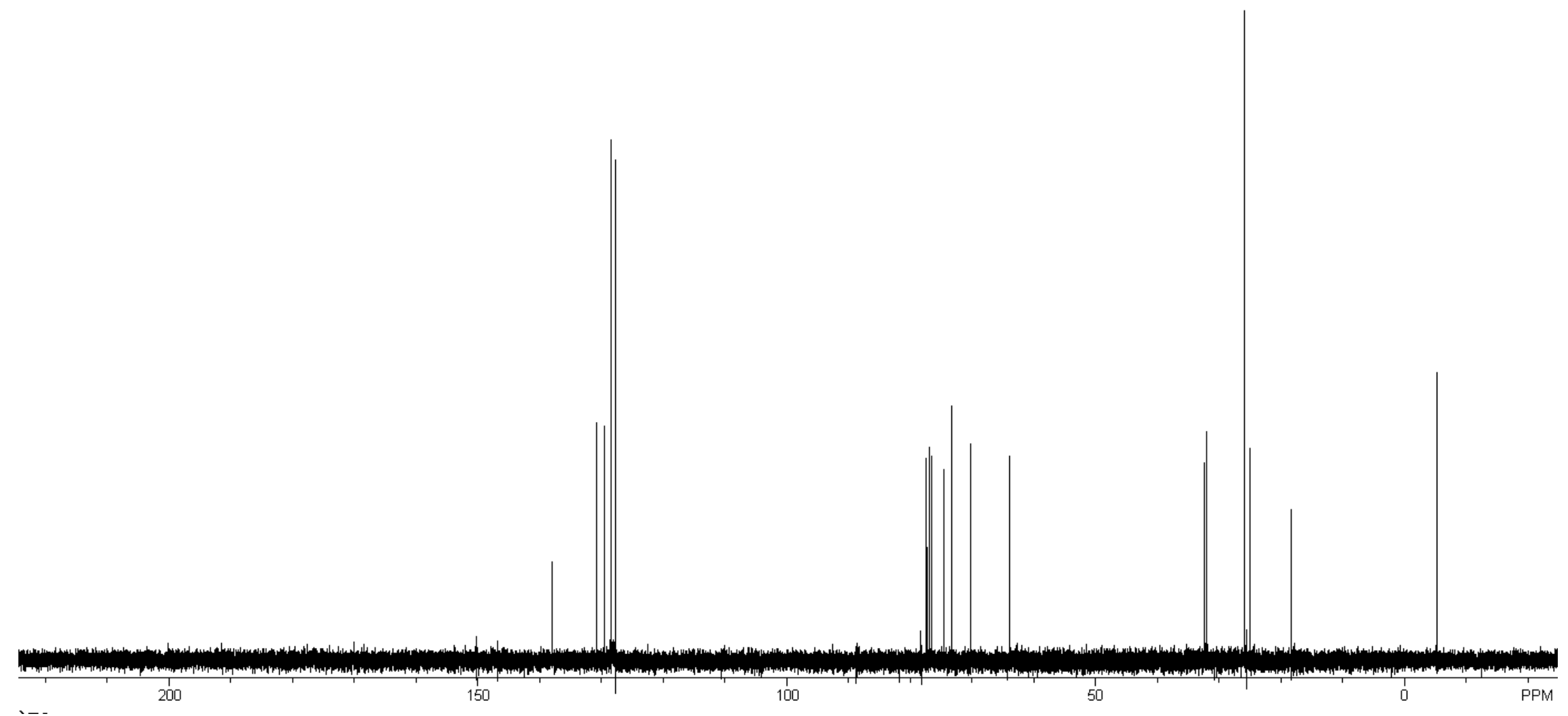


$\underbrace{\mathrm{OH}}_{14} \mathrm{NOH}_{\mathrm{OH}}$

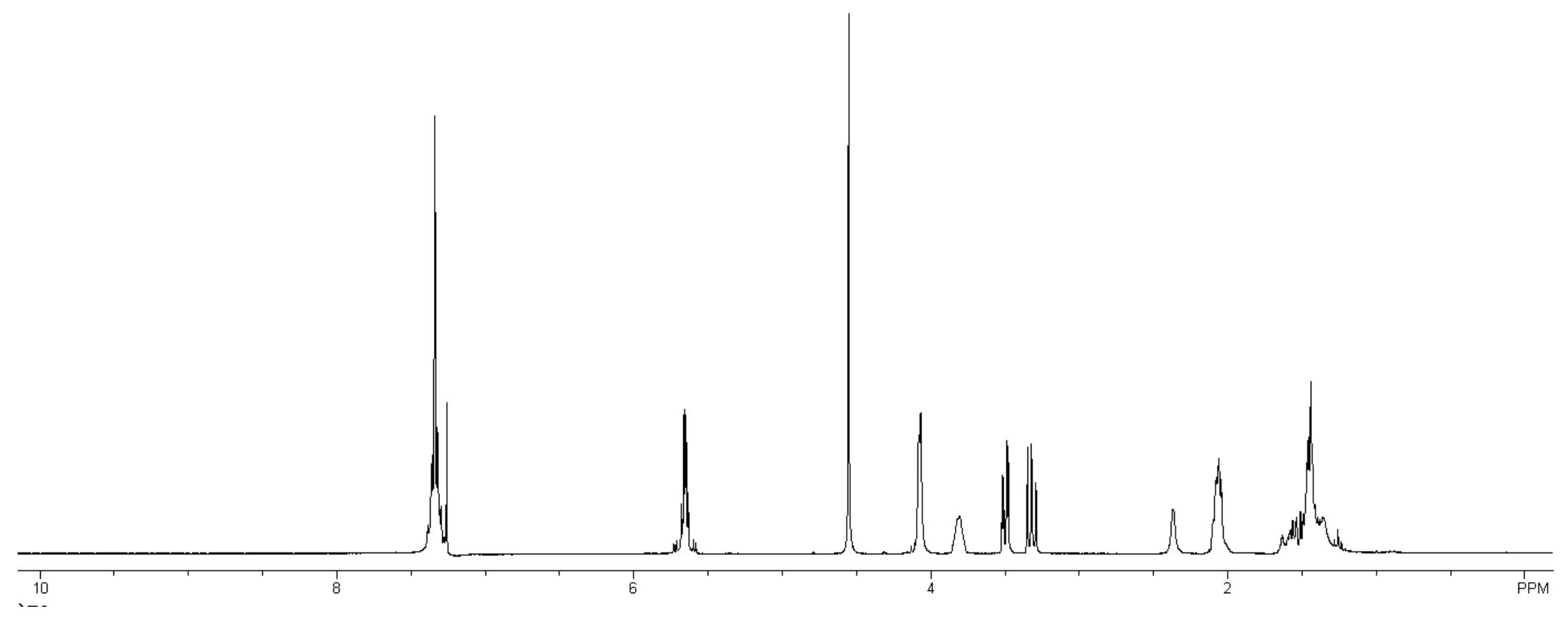


$\mathrm{OH}$

$\mathrm{BnO} / \mathrm{CH}_{14}$

14

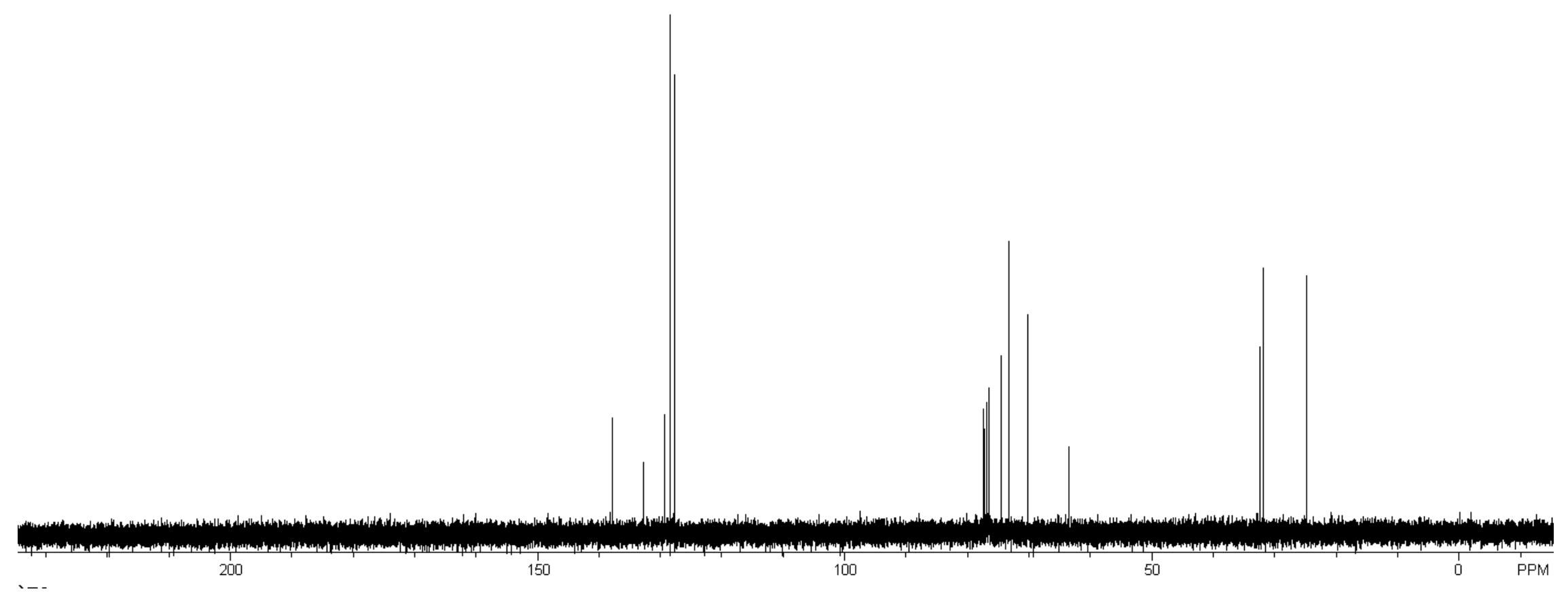


$\underbrace{}_{\mathbf{1 5 a}}$

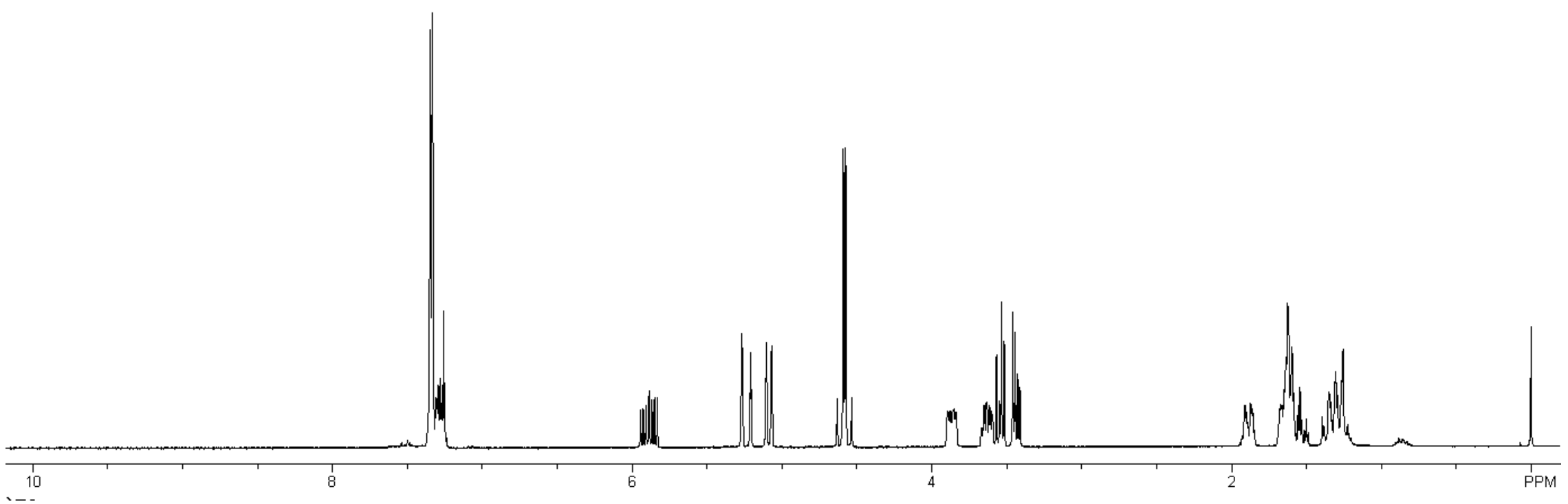


${ }^{\mathrm{BnO}} \mathrm{C}$

$15 a$

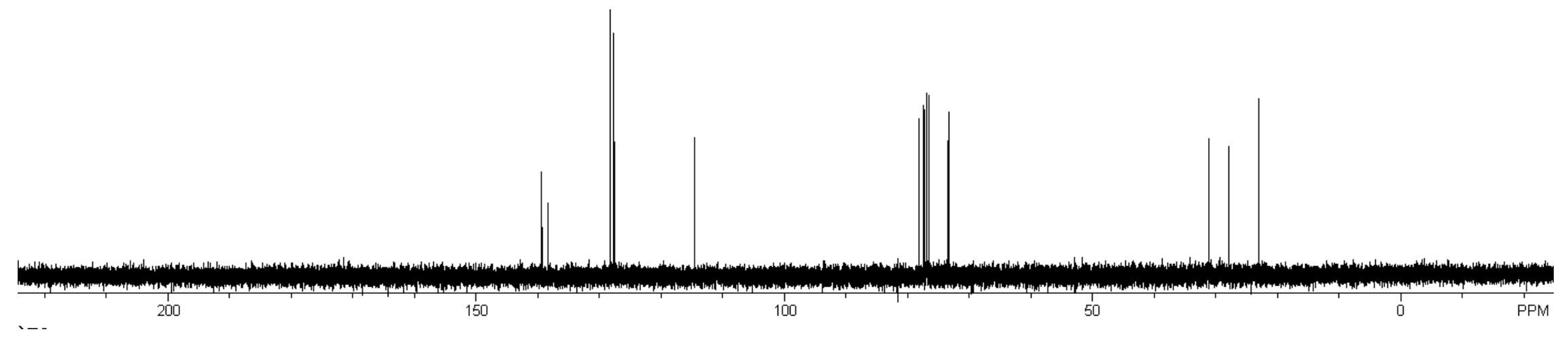


$\underbrace{}_{\mathbf{1 5 b}}$

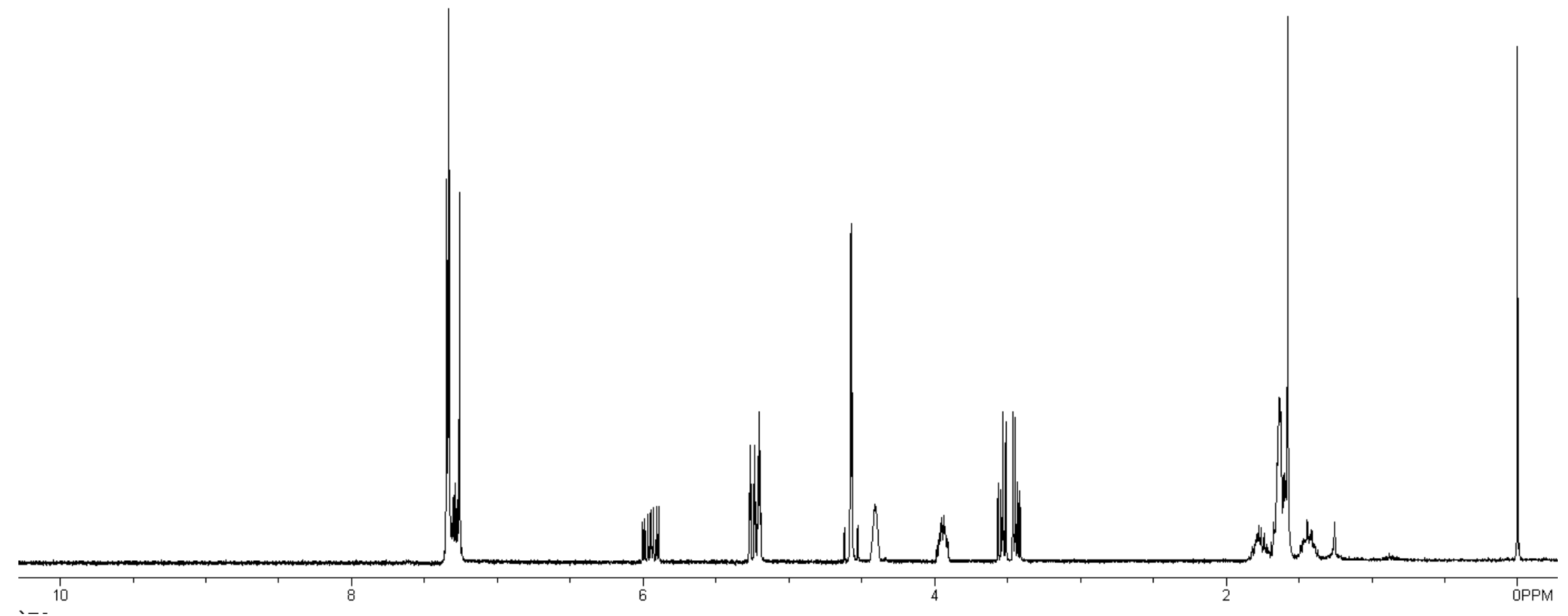


$\sum_{16}^{\mathrm{OH}} \mathrm{OH}_{\mathrm{OH}}$

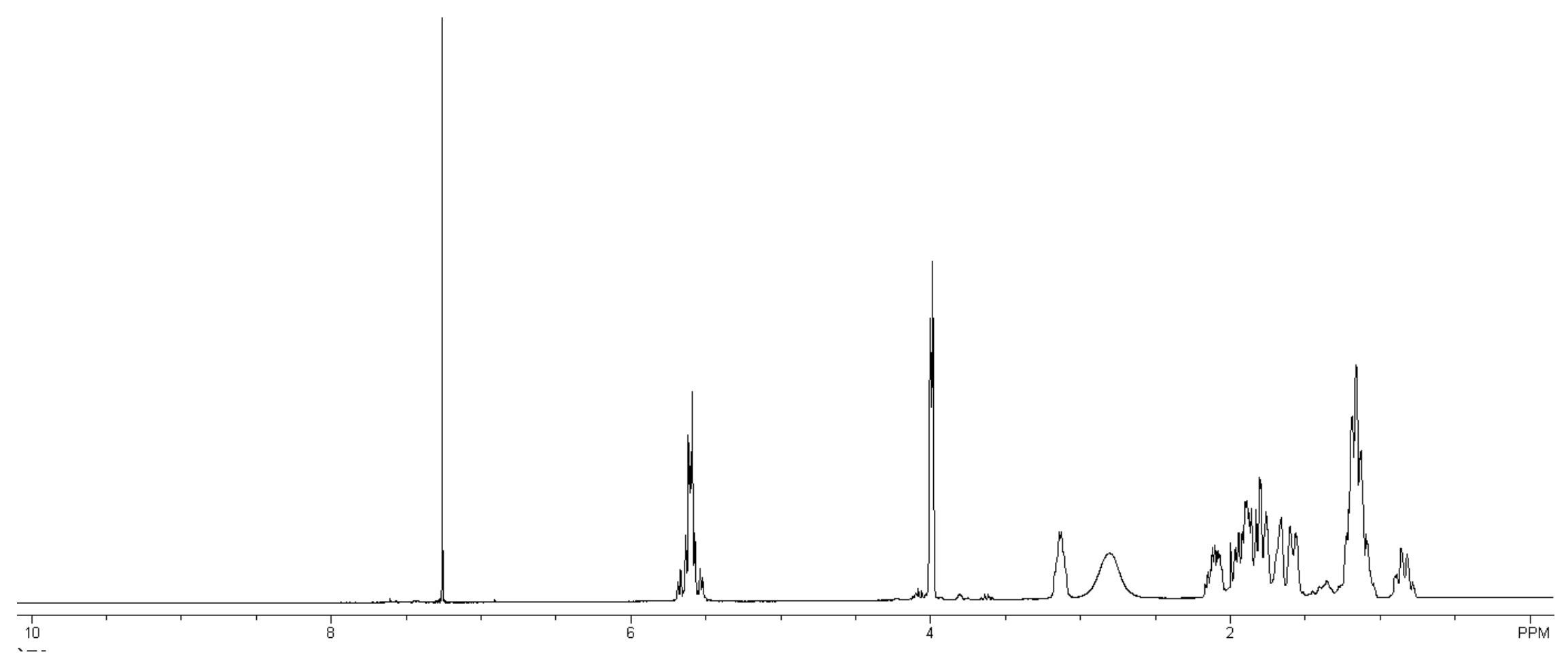


$\bigcup_{16}^{\mathrm{O}} \mathrm{CH}^{\mathrm{OH}}$

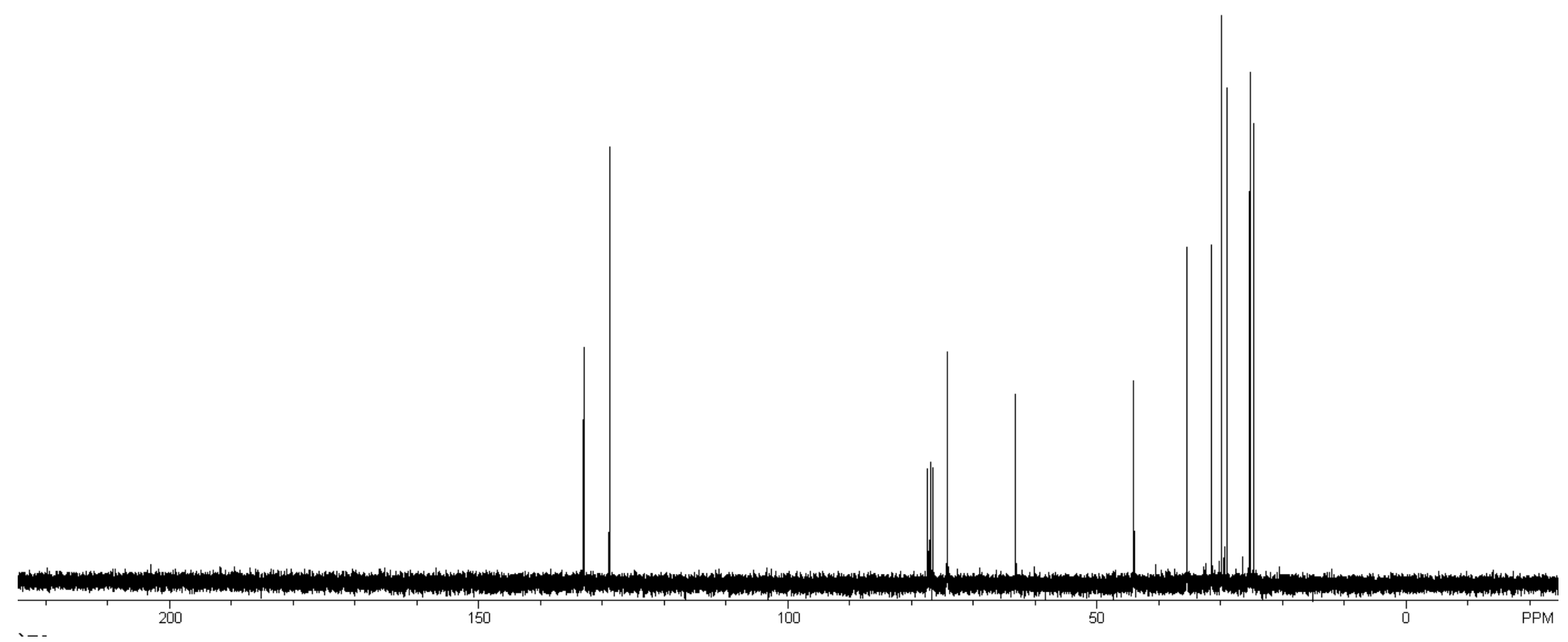


$\overbrace{17 a}^{H}{ }^{H} \curvearrowright$

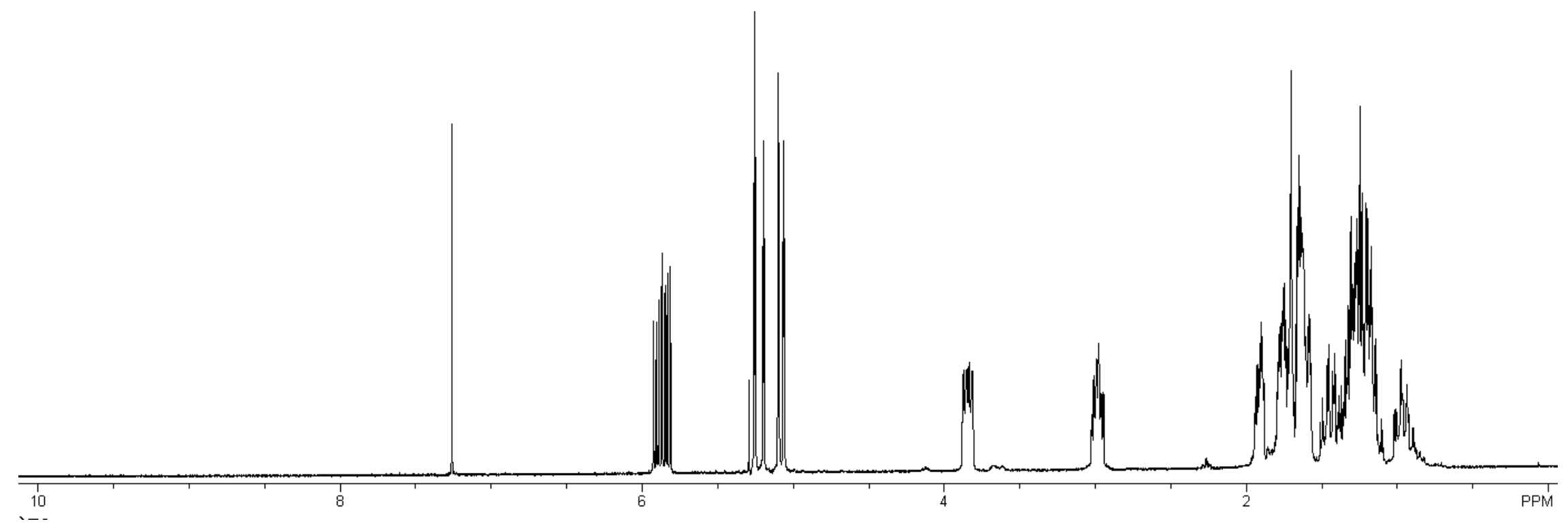



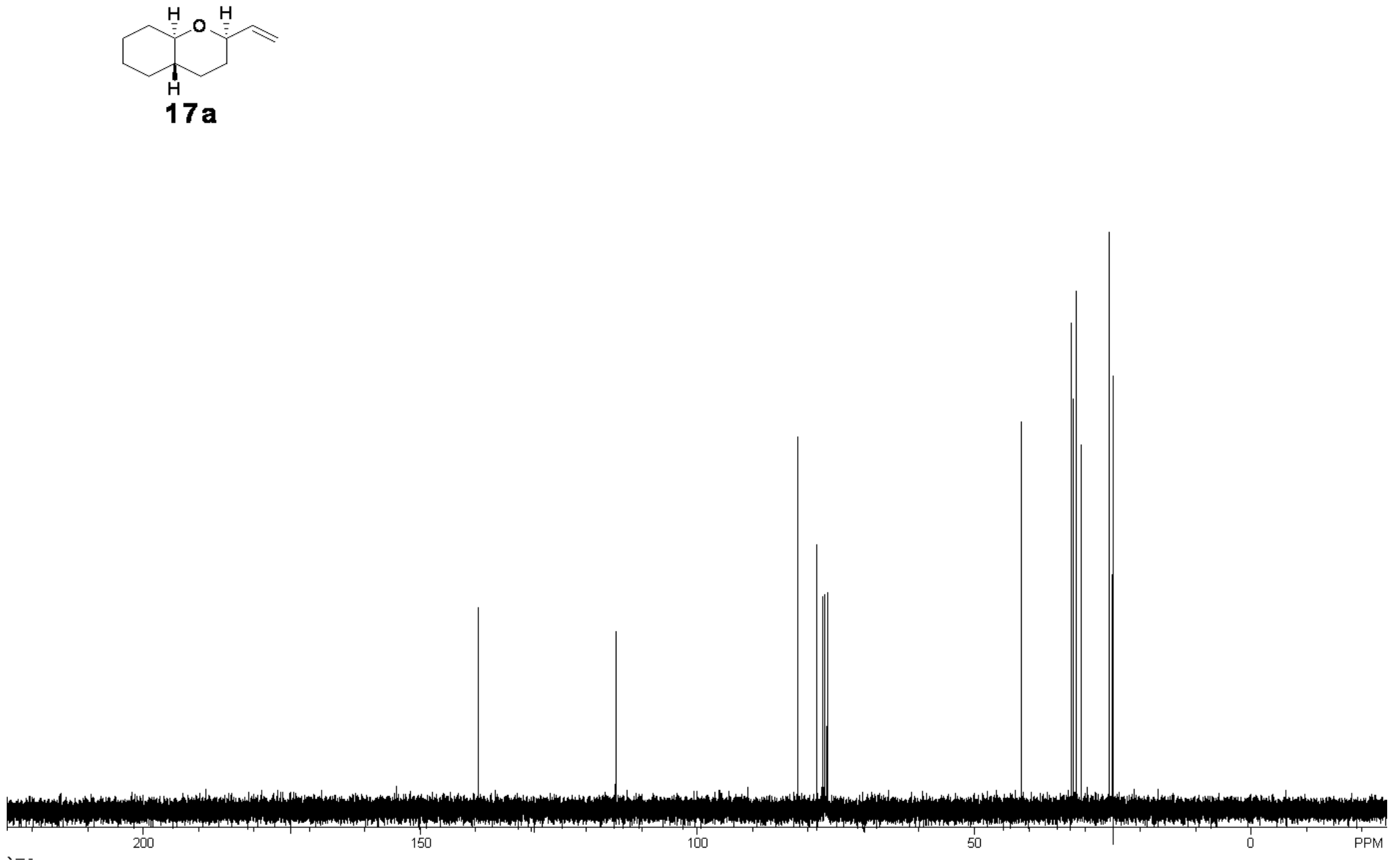


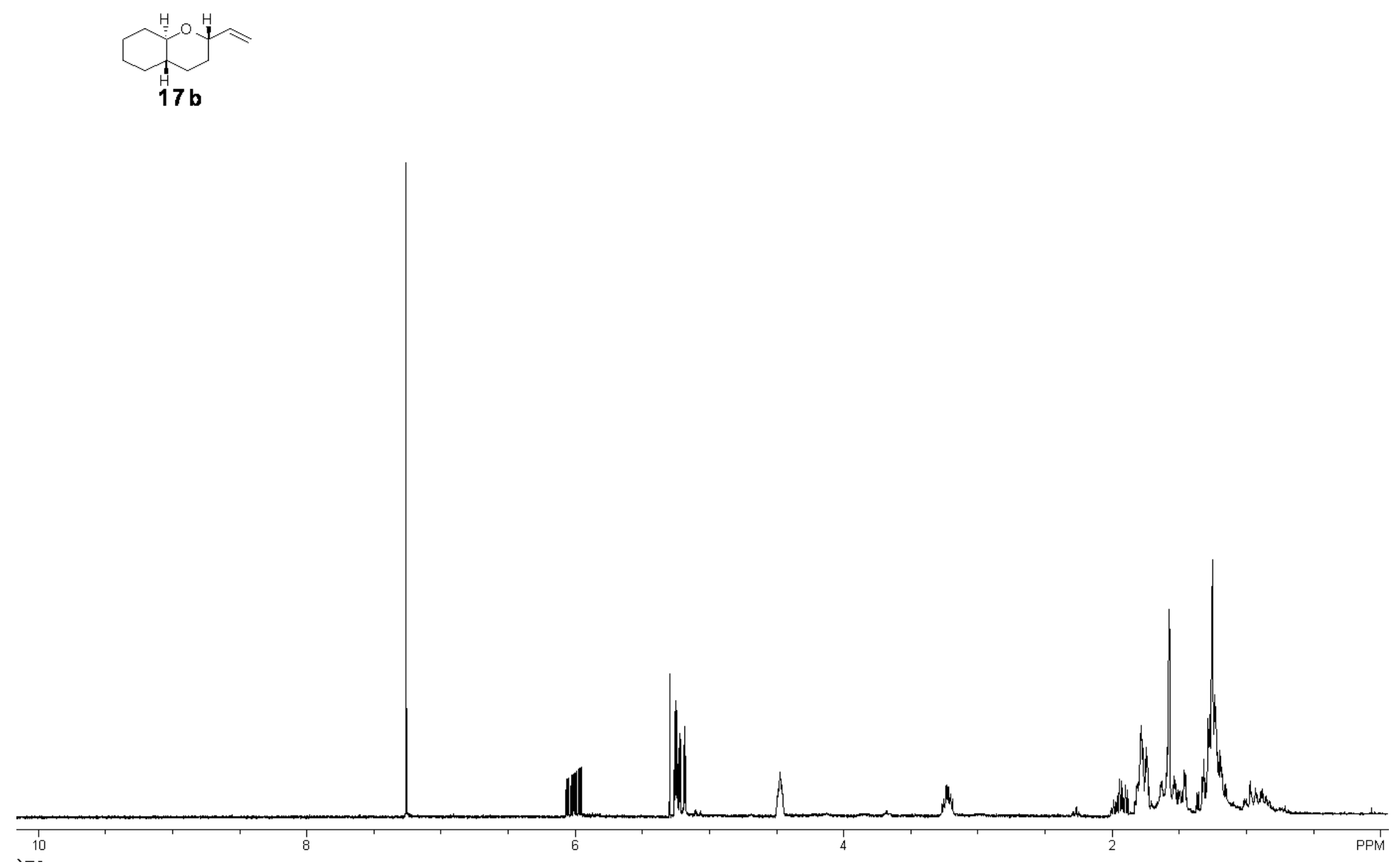




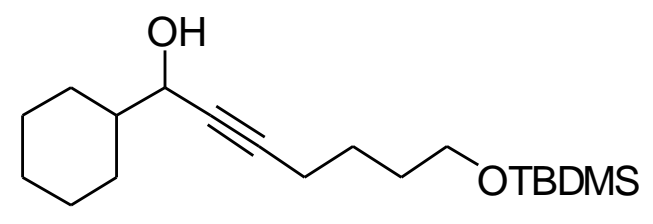

$18 a$

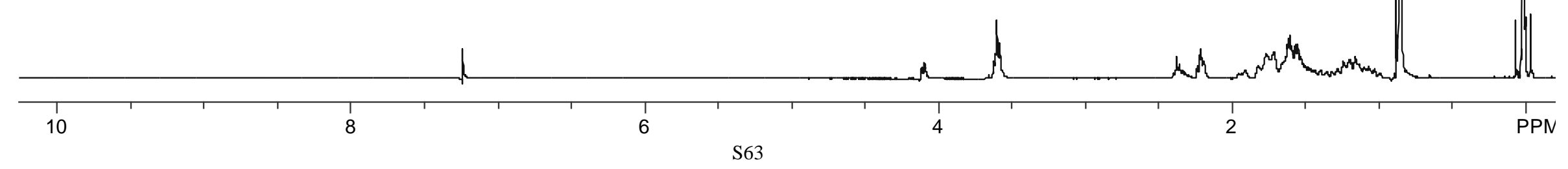




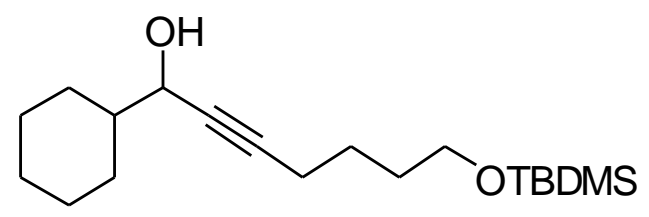

$18 a$

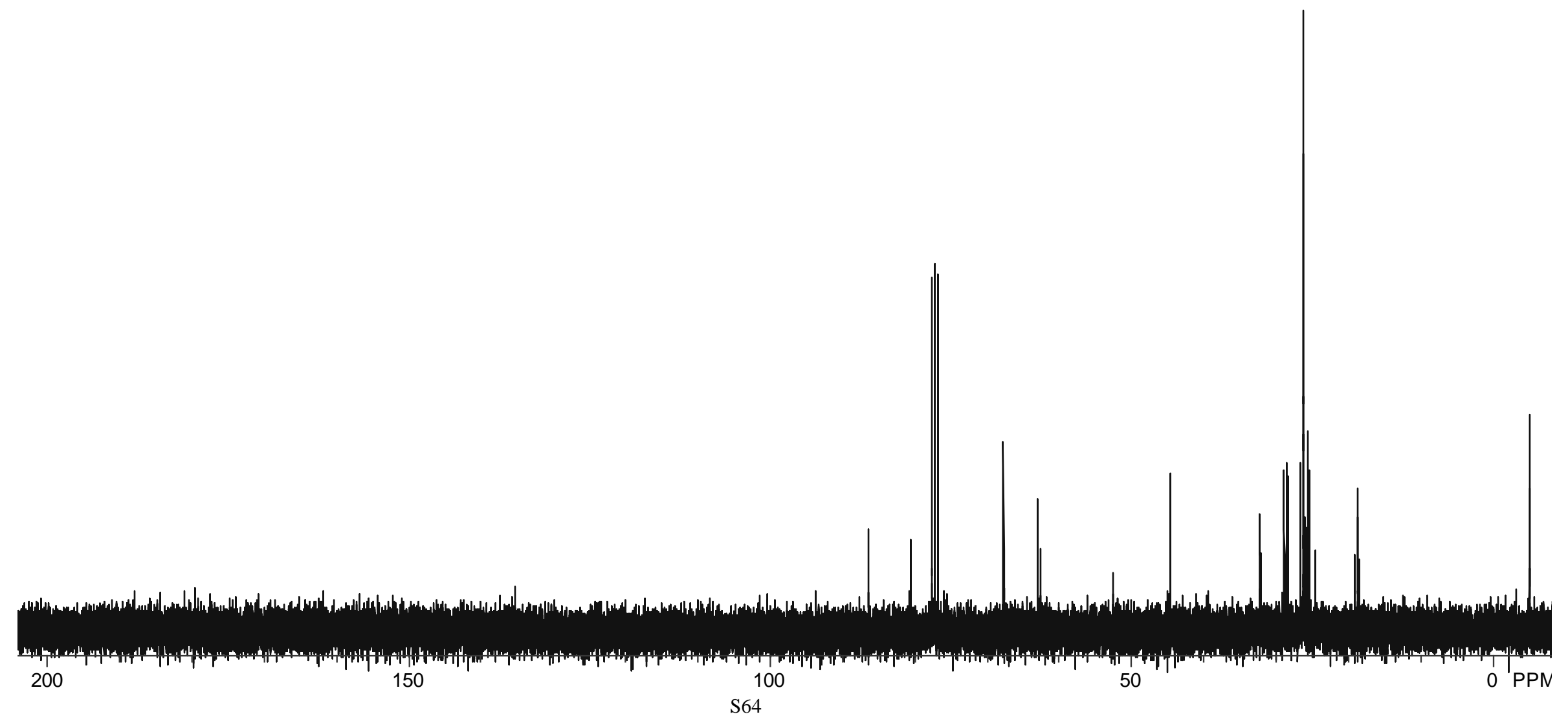



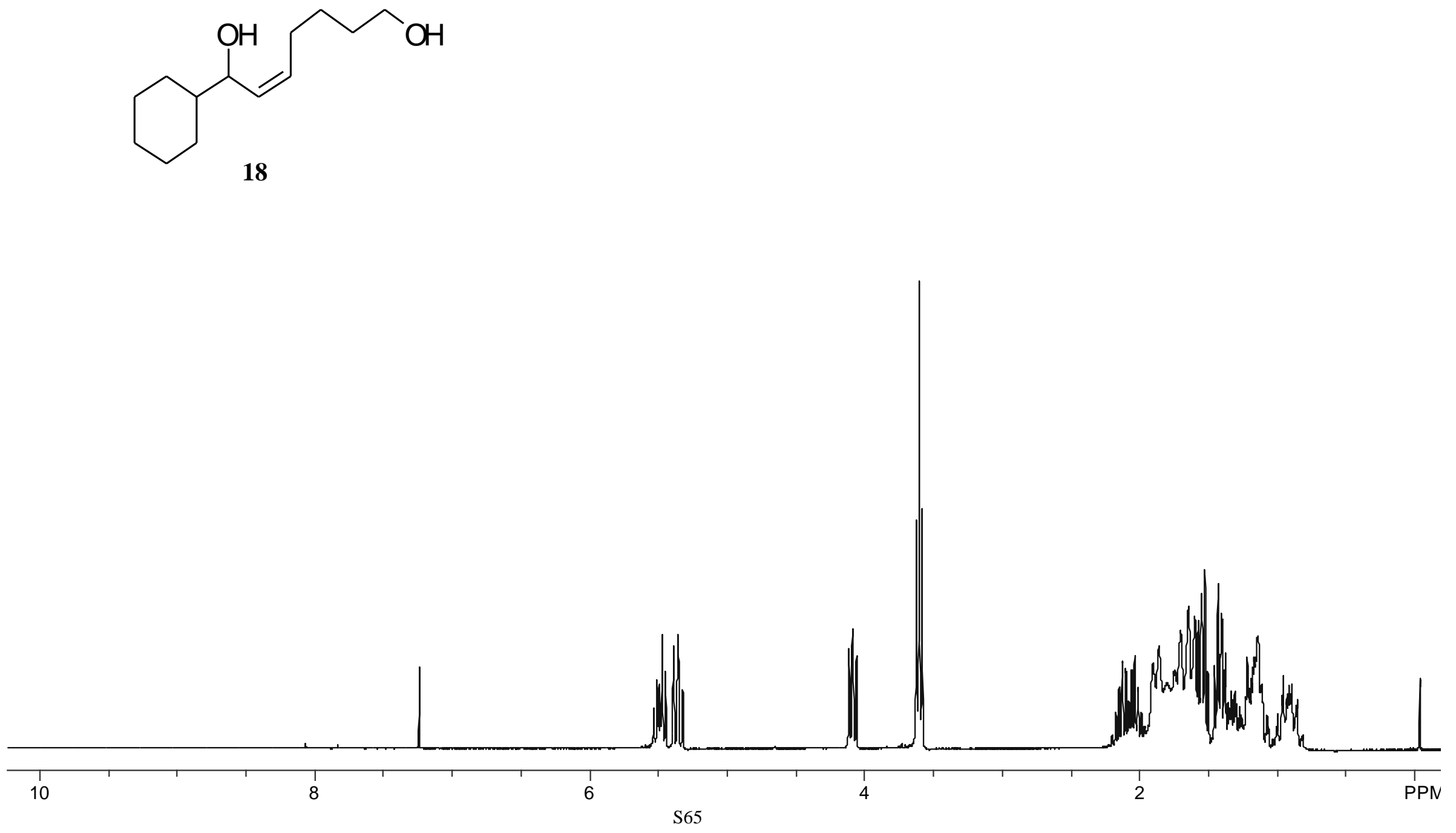


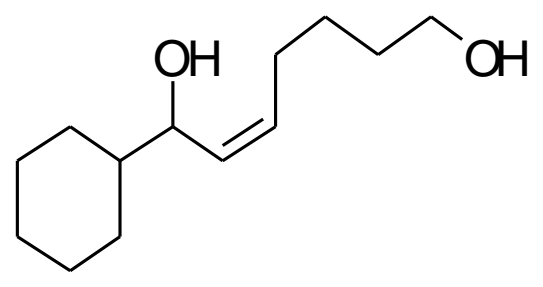

18

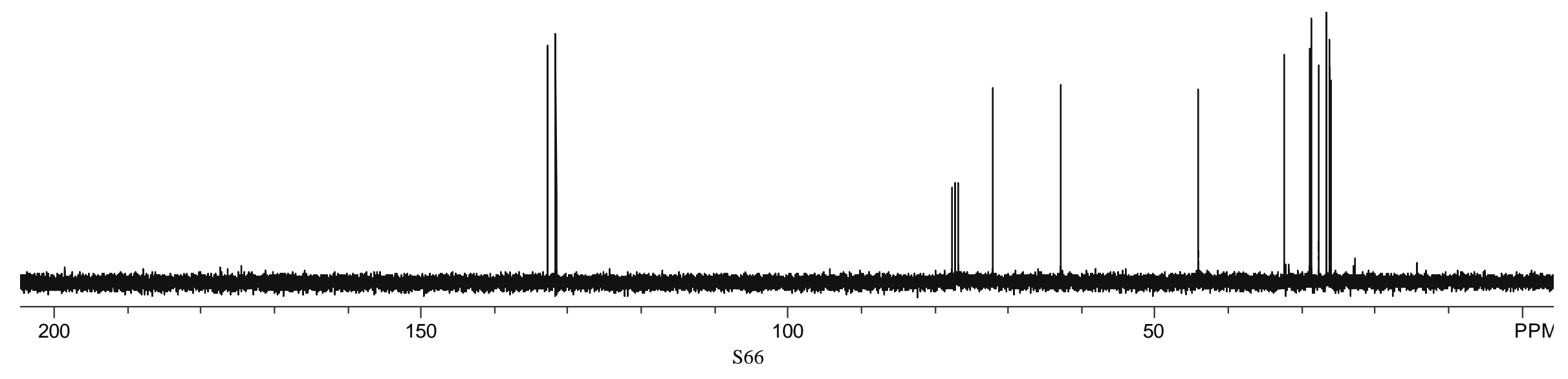


<smiles>OCCCCC=CC1(O)CCCCC1</smiles>

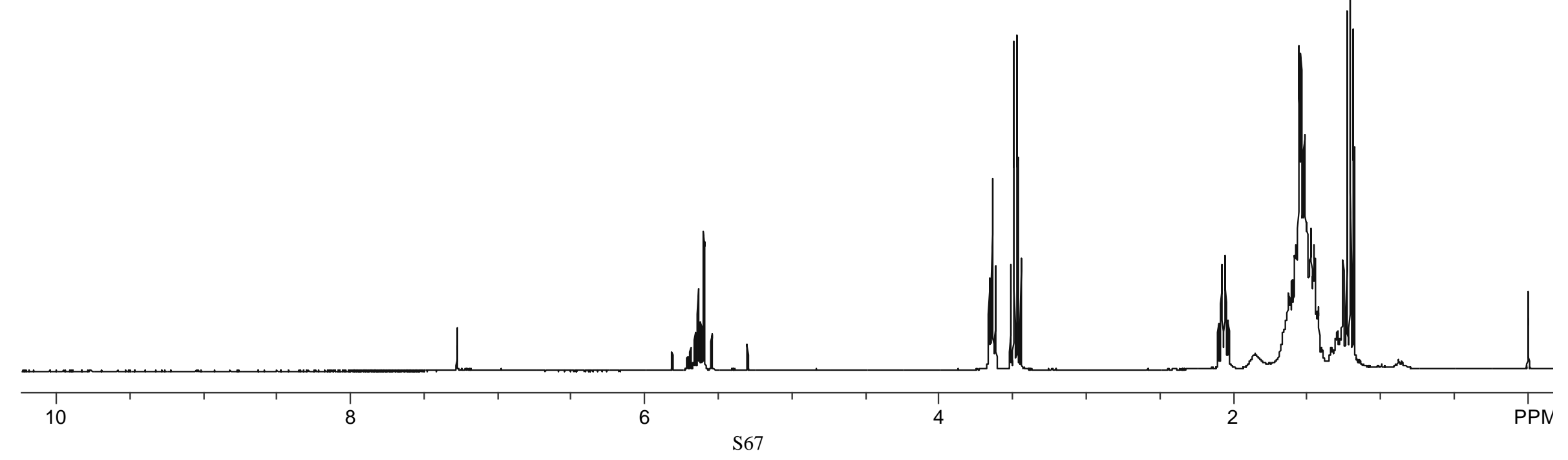



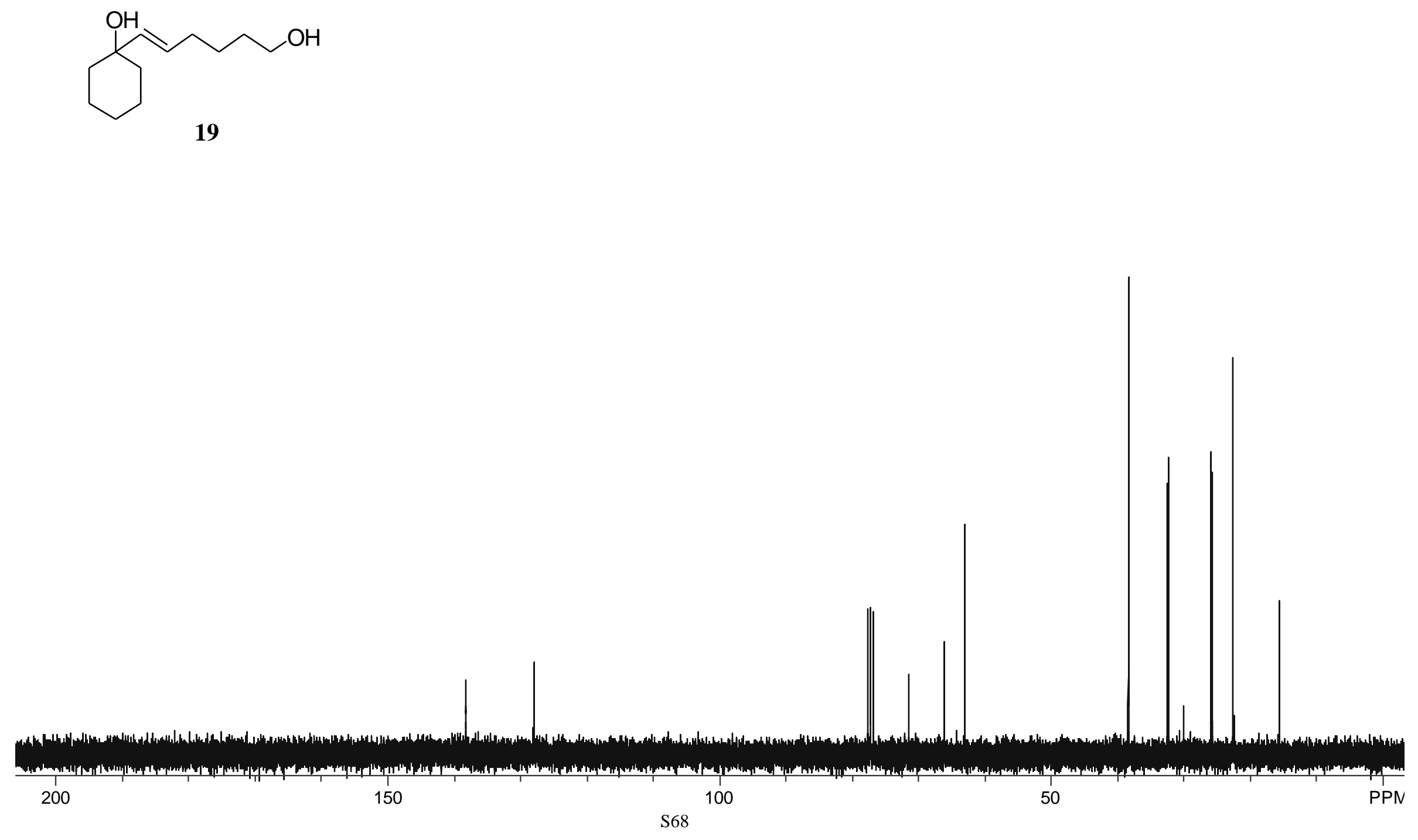


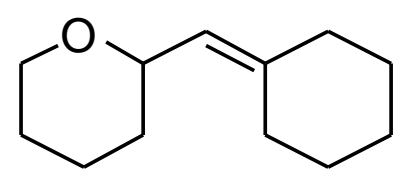

20

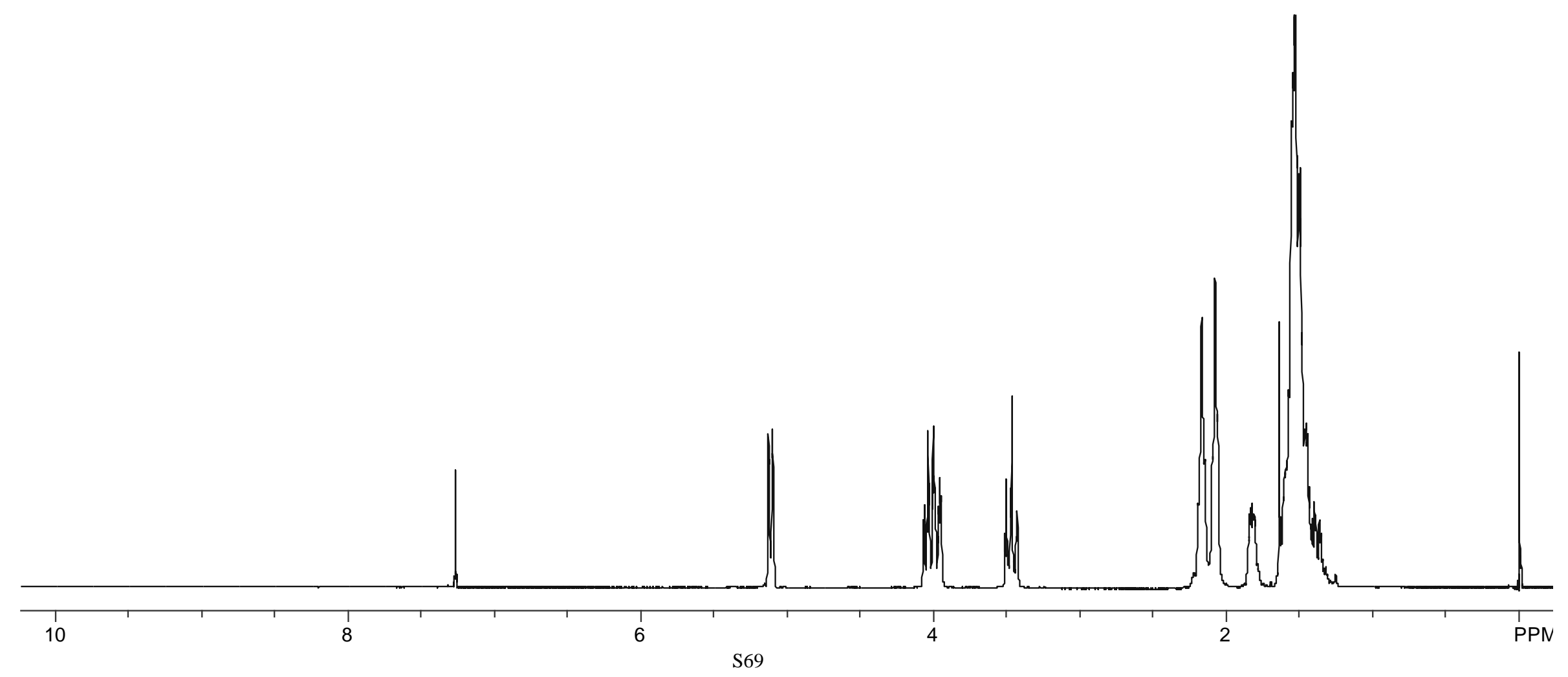




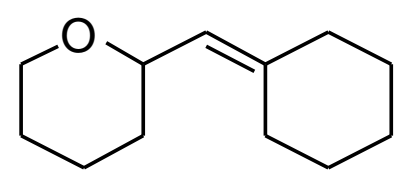

20

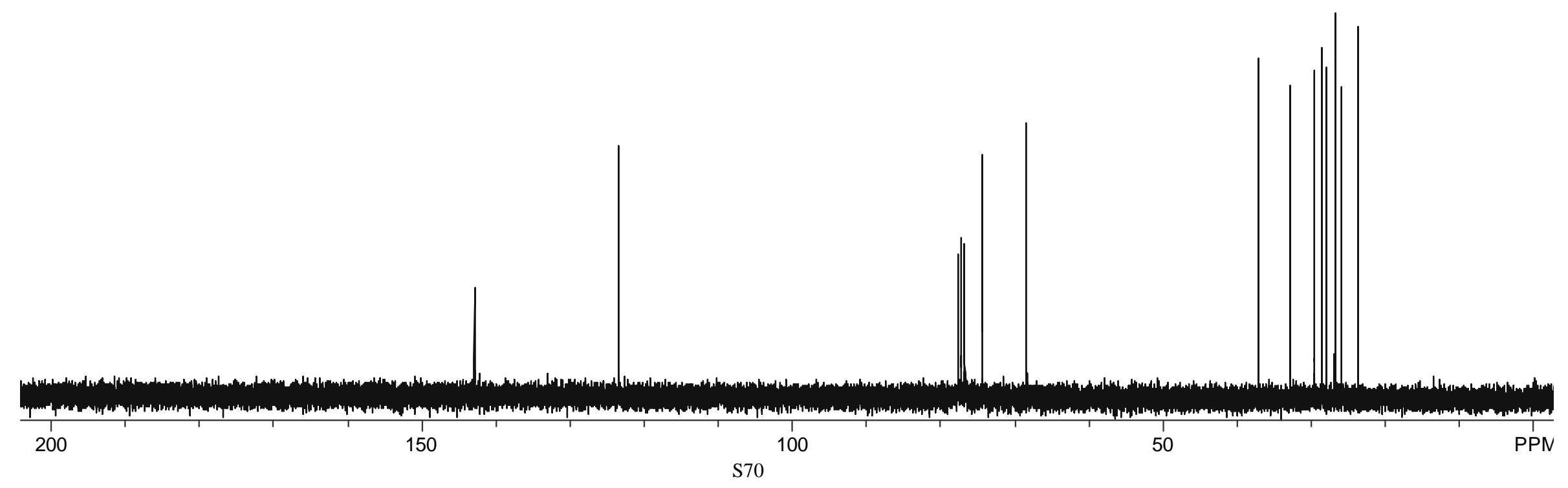




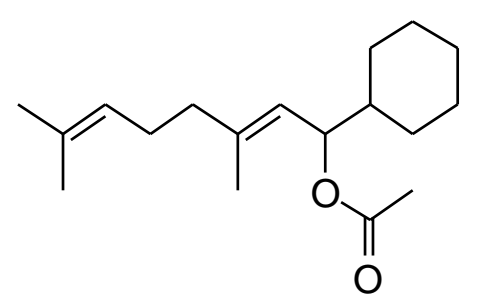

21a

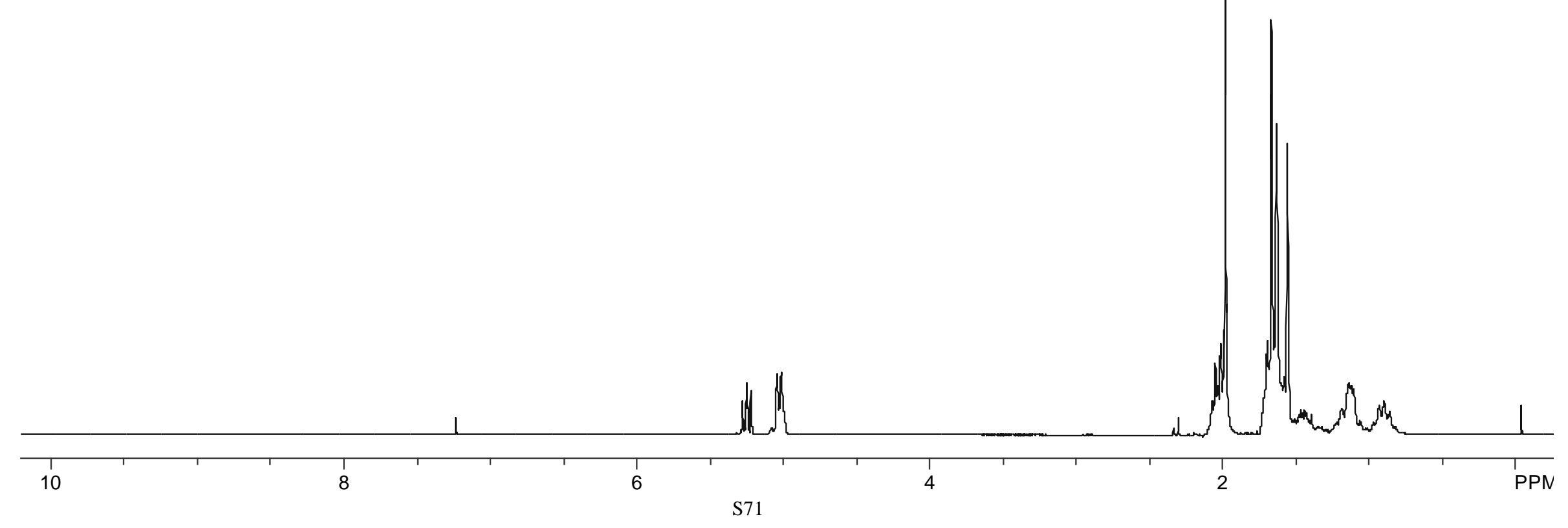



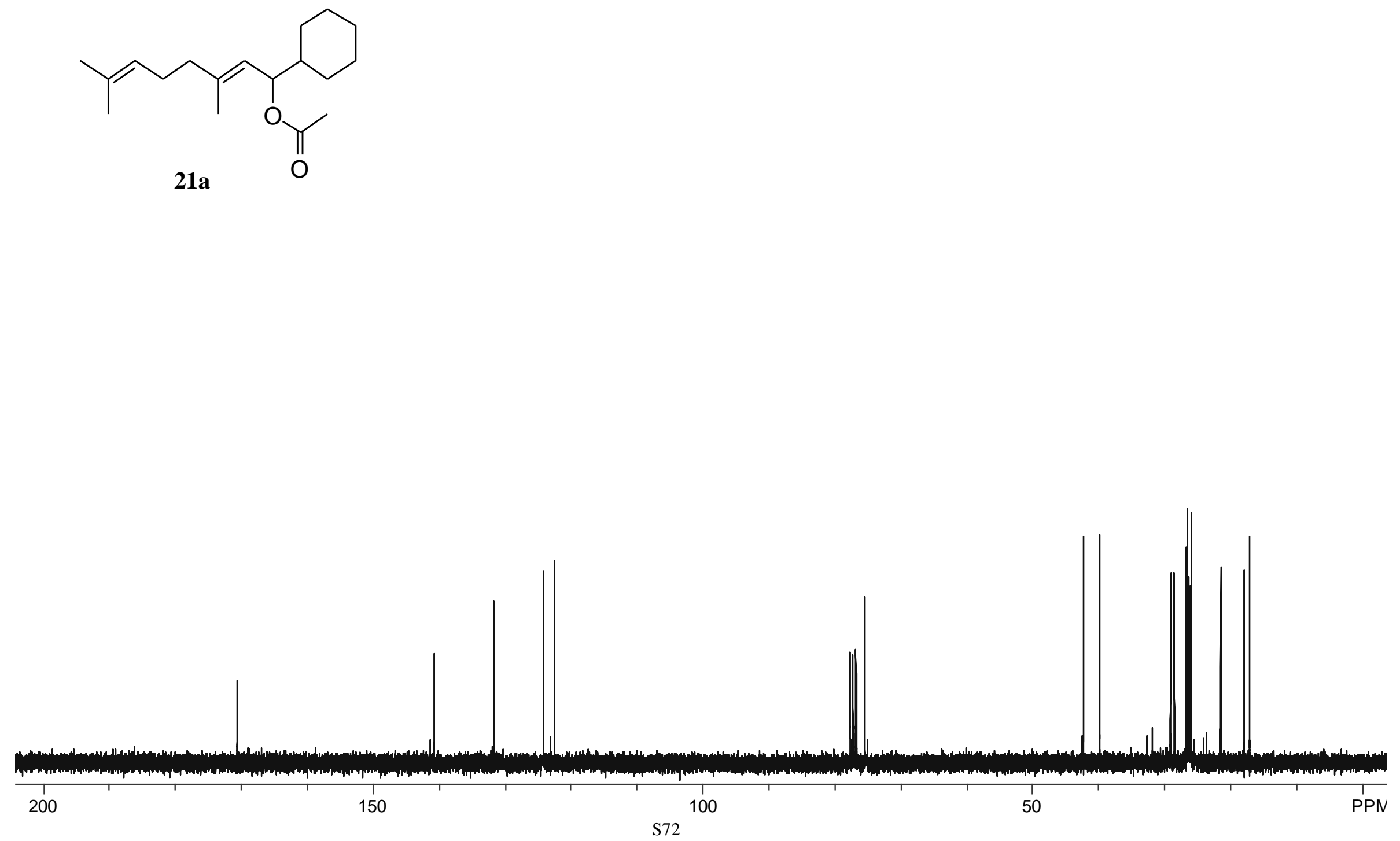


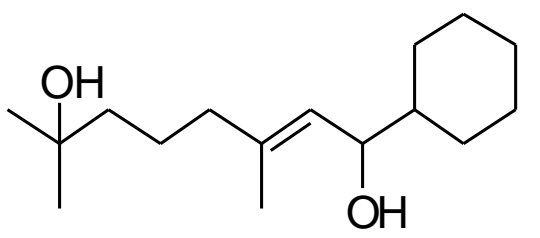

21

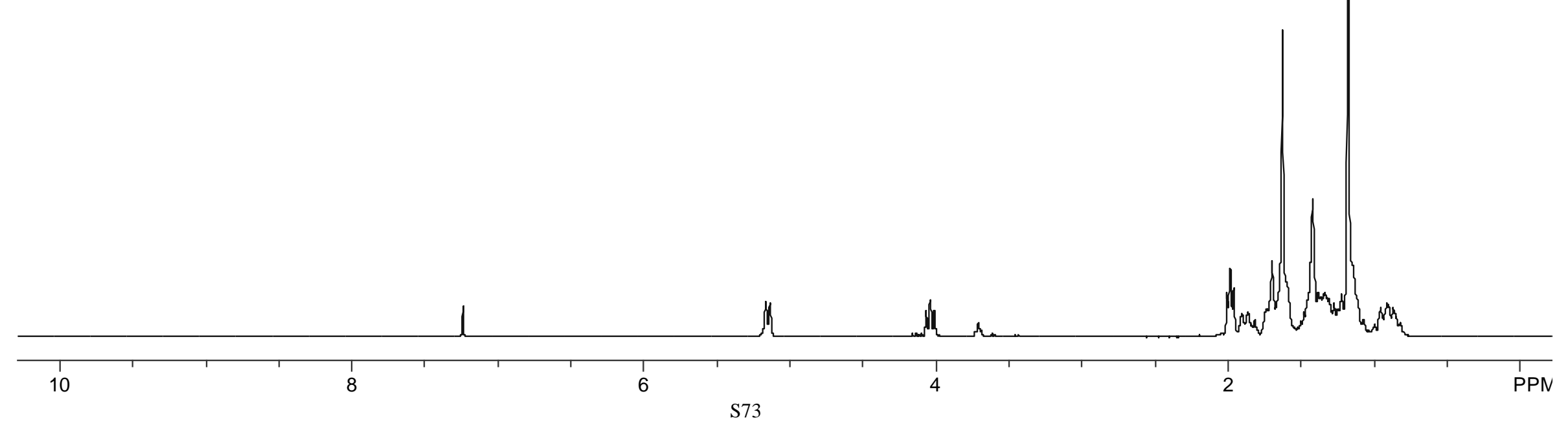




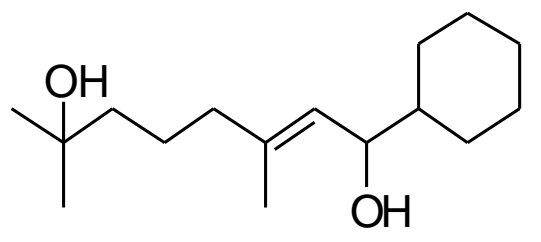

21

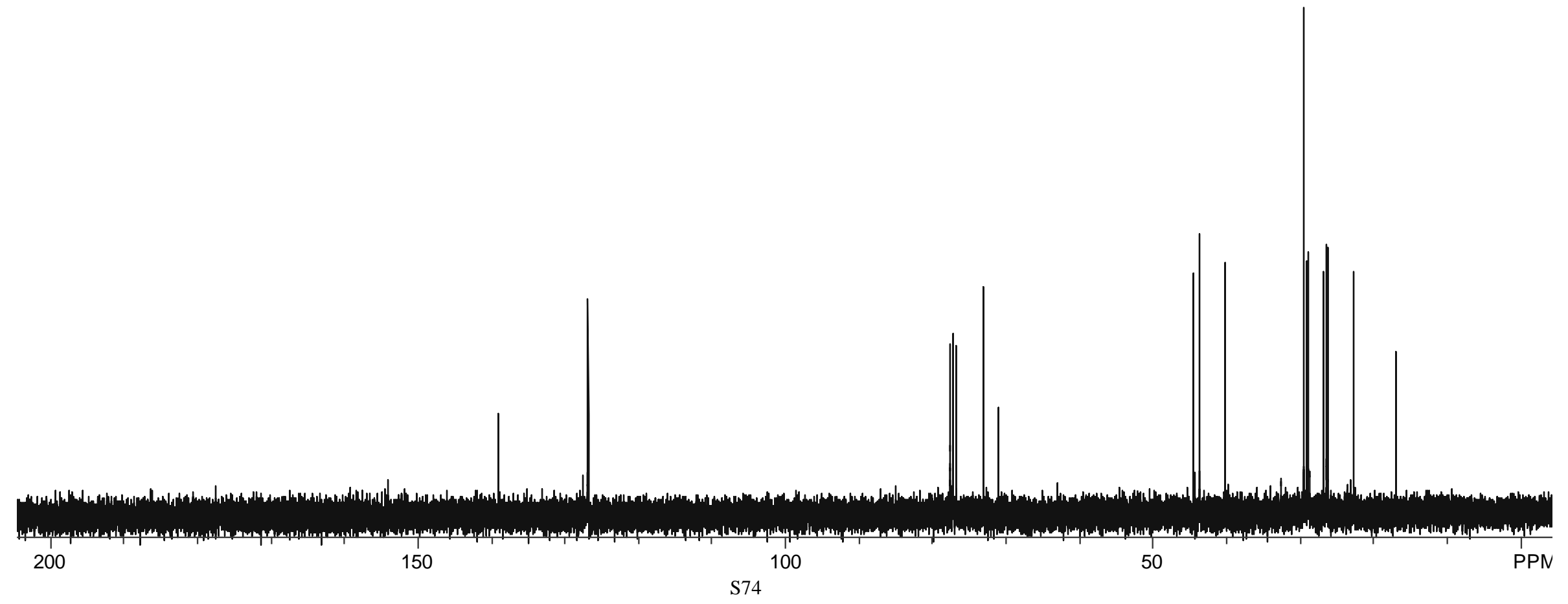




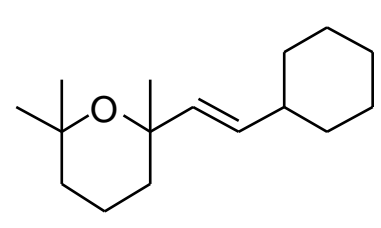

22

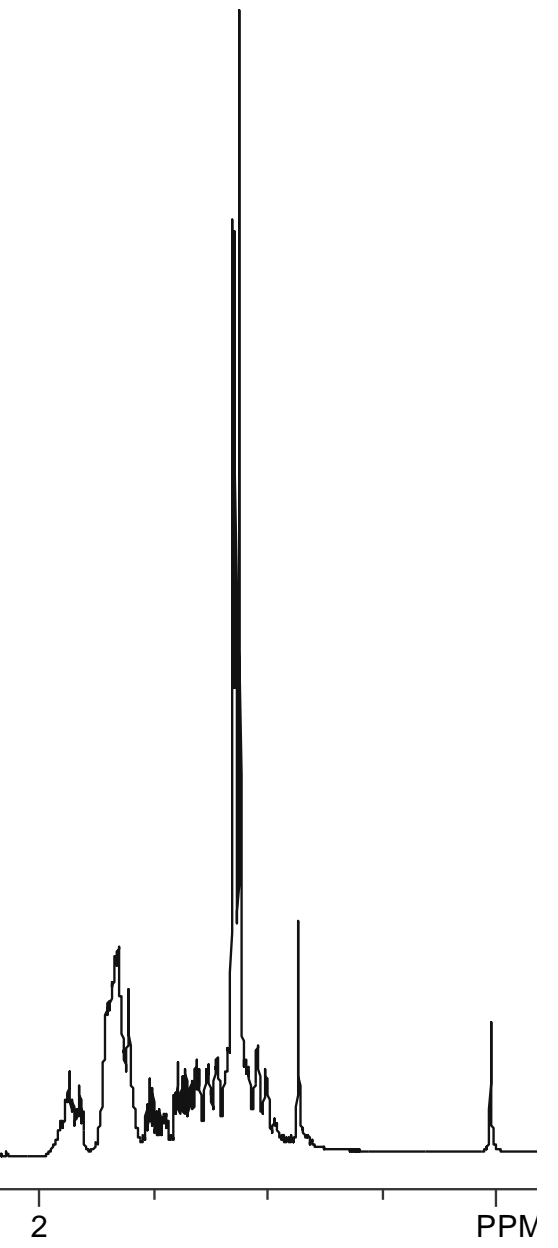




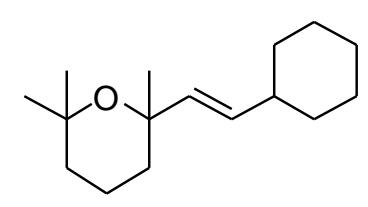

22

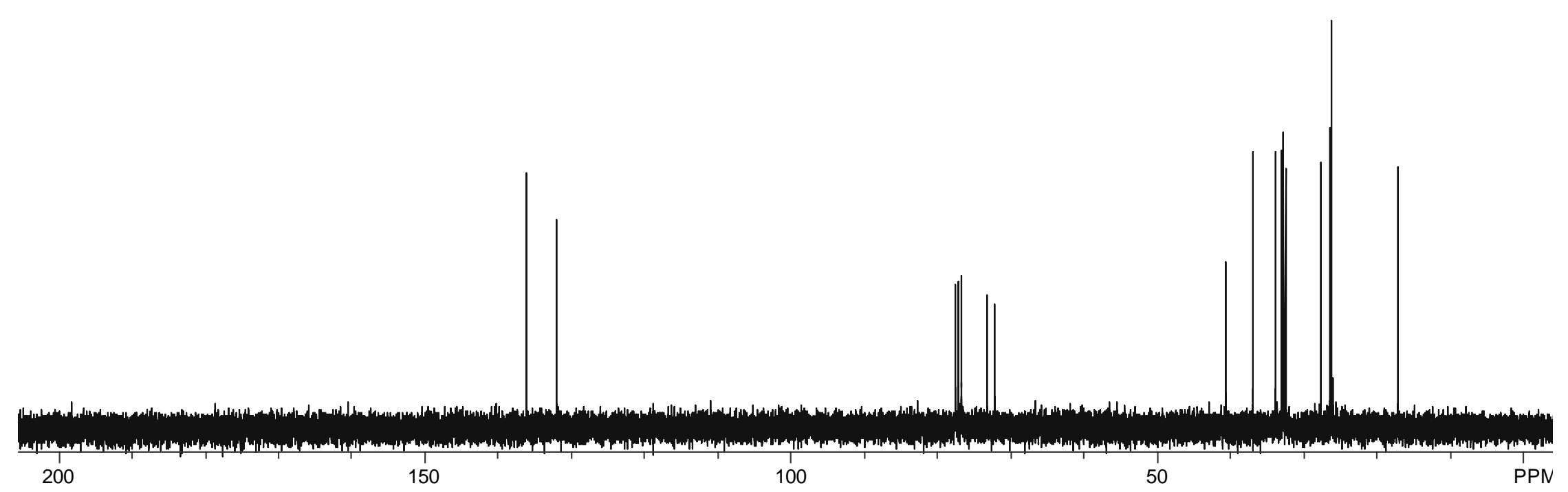

